\title{
RARE GERMLINE VARIANT CONTRIBUTIONS TO MYELOID MALIGNANCY SUSCEPTIBILITY
}

\author{
by
}

SAMUEL TZE KIN LI

Submitted in partial fulfillment of requirements for the degree of Doctor of Philosophy

Dissertation Advisor: Thomas LaFramboise, $\mathrm{PhD}$

Department of Genetics and Genome Sciences

CASE WESTERN RESERVE UNIVERSITY

May 2020 


\title{
CASE WESTERN RESERVE UNIVERSITY SCHOOL OF GRADUATE STUDIES
}

\author{
We hereby approve the thesis/dissertation of \\ Samuel Tze Kin Li
}

Candidate for the degree of Doctor of Philosophy*

Committee chair

David Buchner

\author{
Committee Member \\ Thomas LaFramboise
}

Committee Member

David Wald

Committee Member

Sudha lyengar

Committee Member

Dana Crawford

Date of Defense

May, 2020 
*We also certify that written approval has been obtained for any proprietary material contained therein.

\section{Acknowledgements}

To start off, I would like to thank my advisor Thomas LaFramboise, or Tom. I still remember the day that I received his email 6 years ago saying that I could join his lab, which also marked the starting day of my dissertation. At that time, I knew nothing about programming, not to mention computational biology. Tom tried to teach me as much as he could and now I feel confident about computational biology. I am lucky enough that Tom always stands by me to provide all the assistance that I need, both in science and life.

I want to thank my lab mates for their help and support, especially Meetha, Sneha, Janet and Jianghong. They always gave me help when I most needed.

I want to thank my committee members, Dr. David Buchner, Dr. David Wald, and Dr. Sudha lyengar for their help and input over the years, and for helping to guide this thesis to where it is today

I want to thank my family, my mother, father, brother, sister-in-law, and of course my two smart and cute nephews, who always bring me a lot of joy. Their support is the key reason that I can finish the whole Ph.D. program 
Lastly, I would like to acknowledge my friends in the department for providing encouragement and help with project ideas, which include Stevephen Hung, Chen Weng, Shiyi Yin, Shanshan Zhang, Dean Pontiue, Justine Ngo and lan Bayles.

\section{Contents}

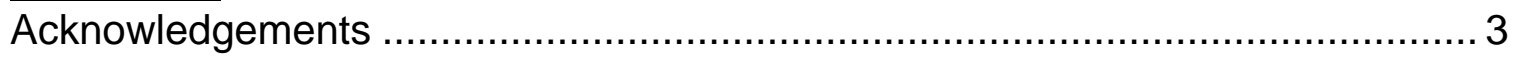

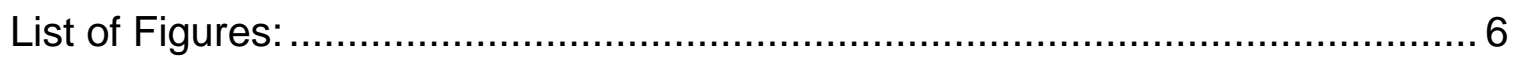

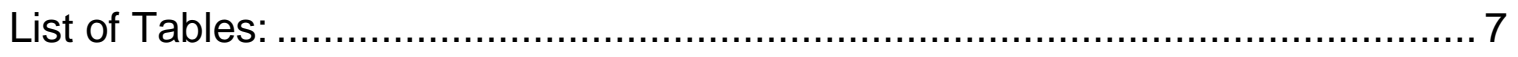

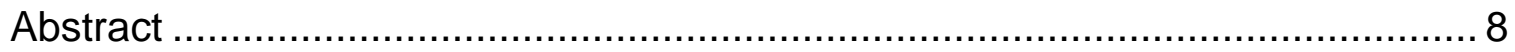

Chapter 1. Background and Significance ................................................. 10

Chapter 1-1: Production of blood cells - Hematopoiesis ................................................ 10

Chapter 1-2: What if things go wrong in hematopoiesis? .............................................. 12

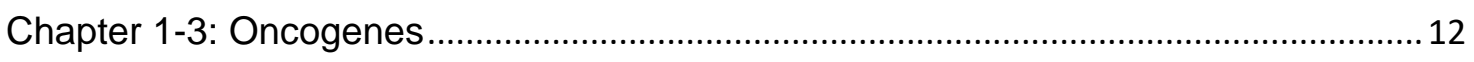

Chapter 1-4: Tumor suppressor genes ............................................................... 13

Chapter 1-5: The two-hit model applies to leukaemogenesis ............................................ 15

Chapter 1-6: The World Health Organization (WHO) classification of AML ..................16

Chapter 1-7: Somatic mutations in myeloid malignancies ............................................... 17

Chapter 1-8: Myeloid Malignancies: Knowns and Unknowns ……................................. 19

Chapter 1-9: The importance of rare variants .............................................................. 22

Chapter 1-10: Difficulties in conducting GWAS in myeloid malignancies ......................23

Chapter 1-11: Rationale for this study ……………………..................................... 24

Chapter 2: Patient collections, variant calling and description of variants ......... 27

Chapter 2-1: Germline whole exome sequencing and ancillary data collection.............27

Chapter 2-2: Raw read processing, variant calling, and quality filtering......................... 71

Chapter 2-3: Variant annotation and variant/gene/patient filtering ...................................73

Chapter 2-4: Filtering putative compound heterozygotes and rare homozygotes ...... 103

Chapter 2-5: Statistical testing ................................................................................. 104

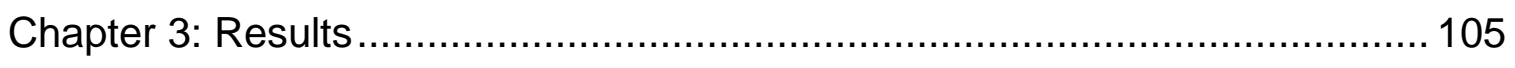


Chapter 3-1: Rare variants in genes with known roles in germline myeloid malignancy

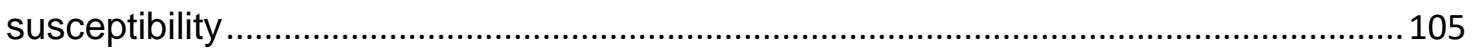

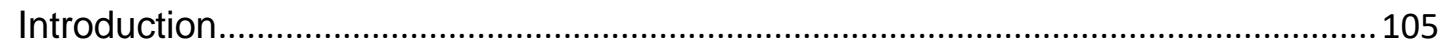

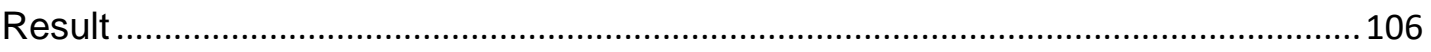

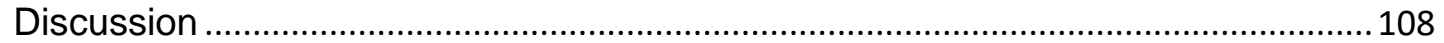

Chapter 3-2: Pathogenic rare variant burden and MPO as a candidate predisposition

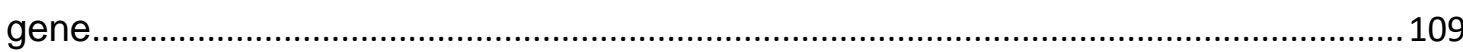

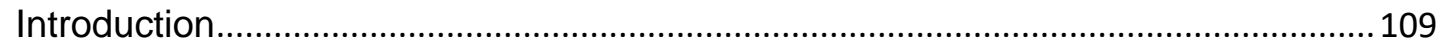

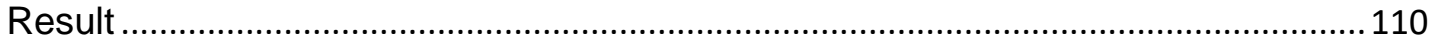

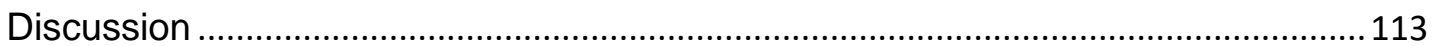

Chapter 3-3: Exploring the overall germline rare variant burden and investigating twohit hypothesis among germline rare variants and with somatic mutations..................114

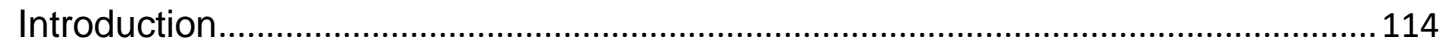

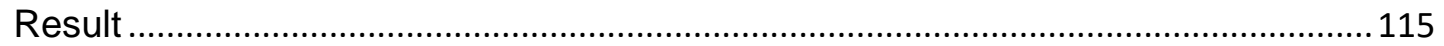

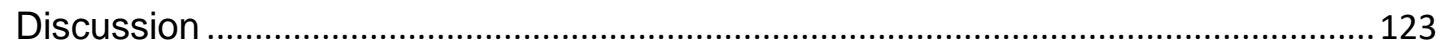

Chapter 4: Discussion \& Future Directions ......................................... 124

The first population-based germline rare variants study of myeloid malignancy......... 124

Whole genome sequencing instead of whole exome sequencing ................................ 125

MPO as a novel myeloid malignancy predisposition gene .......................................... 126

The importance of FA genes in myeloid malignancies............................................... 126

Crosstalk between germline rare variants and somatic mutations in the same patient

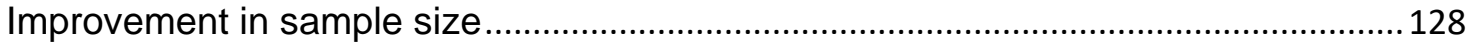

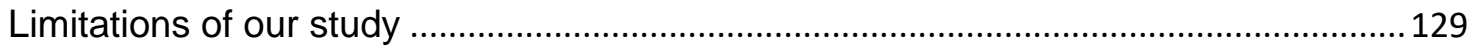

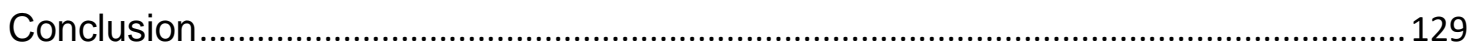

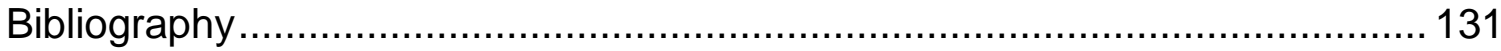




\section{List of Figures:}

Figure 1: Hematopoietic stem cells differentiation pathway ........................................... 10

Figure 2: Description of pipeline to call, annotate, and filter variants from fastq files. ..... 72

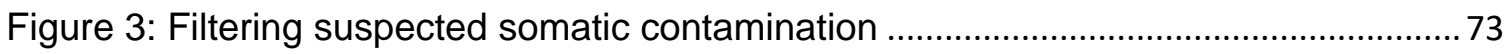

Figure 4: Overview of patient and variant characteristics.............................................. 102

Figure 5: Germline mutations in genes implicated in myeloid neoplasms with germline

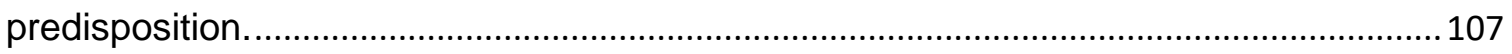

Figure 6: Myeloperoxidase (MPO) as a candidate susceptibility gene in myeloid

malignancy.

Figure 7: Autosomal recessive genes and Fanconi anemia genes have higher overall

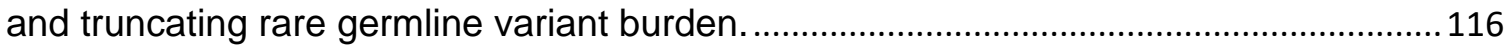

Figure 8: Genes with rare variants in both copies in the same patient...........................119

Figure 9: Global and patient-specific concordance of rare germline variants and somatic

mutations in each gene. 


\section{List of Tables:}

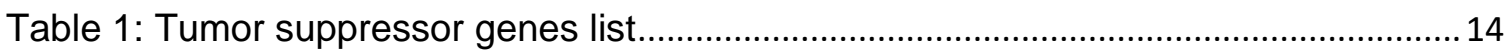

Table 2: Common cytogenetic abnormalities in AML .................................................. 16

Table 3: Genes which are recurrently somatically mutated in myeloid malignancies

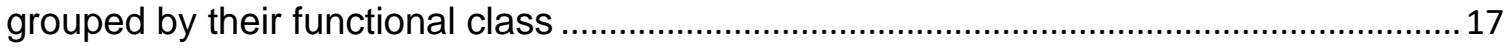

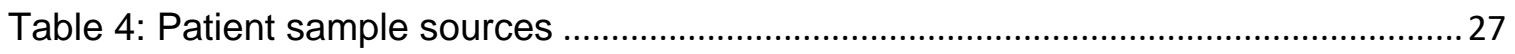

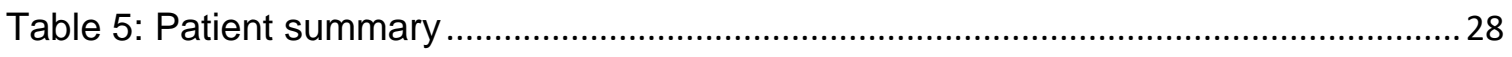

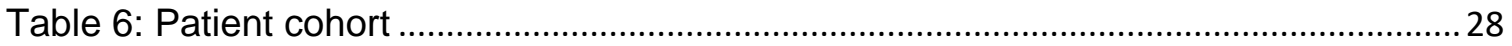

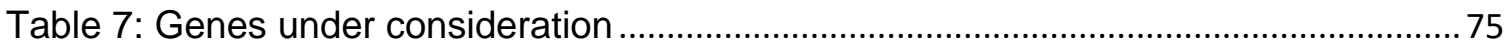

Table 8: Classification of myeloid neoplasms with germ line predisposition ....................105

Table 9: Number of whole-genome and whole-exome patient samples available from

public sources 


\title{
Rare Germline Variant Contributions to Myeloid Malignancy Susceptibility
}

\author{
Abstract \\ By

\section{SAMUEL TZE KIN LI} \\ Myeloid malignancies are rare but severe clonal disorders found in hematopoietic \\ stem or progenitor cells. Both genetic and epigenetic changes can contribute to \\ the disease by disturbing some key aspects in cells such as self-renewal, \\ proliferation and differentiation. Therefore, it is important to know which genes are \\ associated with these diseases in order to increase our understanding assess \\ disease risk and develop therapeutic strategies to treat the disease. Recurrent \\ somatic mutations in some genes, such as $R U N X 1, I D H 1 / 2$ and $F L T 3$, are known \\ to be associated with development of these diseases. However, in finding the \\ association between genes and myeloid malignancies, there is still a question as \\ to the degree to which germline rare variants contribute to the disease phenotype.
}


In chapter 3, we will present our findings from 690 myeloid malignancies patients' whole-exome sequence data to elucidate the contribution to disease susceptibility of rare $(<1 \%$ population allele frequency) protein-altering alleles. We estimate that $3-4 \%$ of patients have rare variants in genes that would classify the patients as having germline predisposition under current guidelines. We also identify MPO as a new candidate predisposition gene, with 19 patients $(2.8 \%)$ harboring rare pathogenic MPO variants, and significantly higher pathogenic allele frequencies in cases as compared to population controls. An elevated rare-variant burden is observed in autosomal recessive genes, particularly Fanconi anemia (FA) genes. The proportion of rare variants that are truncating is substantially higher in autosomal recessive and FA genes than in other. Even after accounting for numbers of rare variants, inherited pairs of such variants in the same patient and same FA gene (presumably affecting both parental homologs) are observed at a rate significantly higher than would be expected by chance, suggesting biparental inheritance of rare alleles in FA genes as a risk factor for myeloid malignancy. The FA gene $B R C A 2$ is particularly enriched for biparental inheritance, and rare germline BRCA2 variants are associated with poor overall survival. 


\section{Chapter 1. Background and Significance}

\section{Chapter 1-1: Production of blood cells - Hematopoiesis}

Hematopoiesis refers to the normal blood cell production. It is an important pathway as this process produces the majority of the cells in human body. Blood cells originate with pluripotent stem cells, which have the ability to both differentiate into other types of stem cells and renew as the same type of cell. Pluripotent stem cells can then differentiate into hematopoietic stem cells (HSCs), which are the origin of all types of blood cells. This type of cell also has the capability for renewal. Notably, about 1 in every 10,000 to 15,000 bone marrow cells is thought to be a stem cell while this proportion falls to 1 in 100,000 blood cells outside bone marrow (National Institutes of Health, 2016).

Figure 1: Hematopoietic stem cells differentiation pathway 


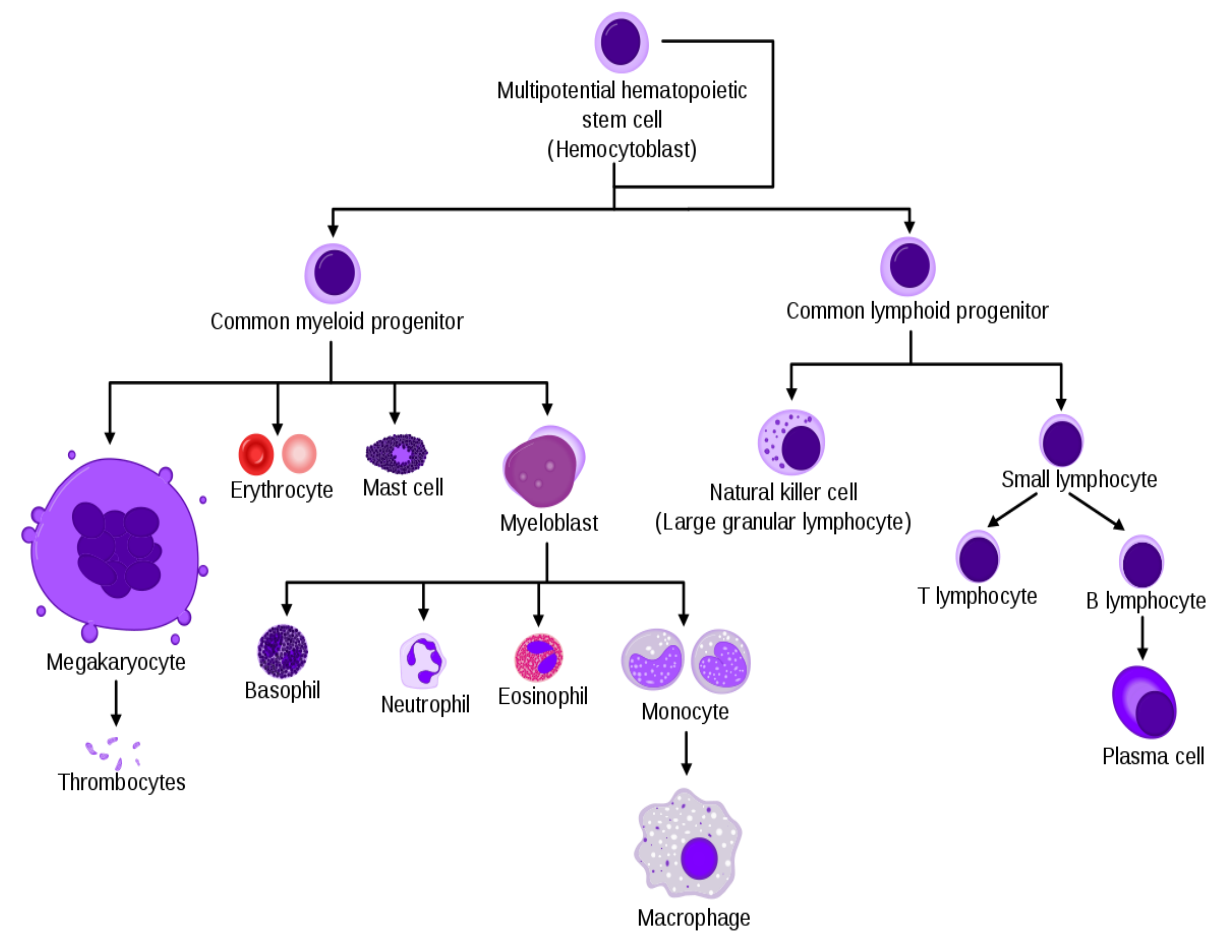

Figure 1: Overview of Hematopoietic stem cells differentiation pathway. Adapted from (Pirahmadian, 2016)

HSCs can then both create more HSCs and differentiate themselves into the myeloid lineage or lymphoid lineage of blood cells. In the myeloid lineage, common myeloid progenitor (CMP) cells can then differentiate into different myeloid precursor cells, which are known as blast cells, such as megakaryoblasts, myeloblasts and proerythroblasts. The myeloblast progenitors can further differentiate into various types of mature blood cells including eosinophils, basophils, neutrophils and monocytes. On the other hand, the lymphoid lineage is started with common lymphoid progenitor (CLP), and it can renew itself and further differentiate into either B-cells or T-cells through the lymphocyte precursor (Harrison, 1982) (Figure1). 


\section{Chapter 1-2: What if things go wrong in hematopoiesis?}

Blood cancers arise if any of the differentiation steps goes wrong during hematopoiesis. There are three main types of blood cancers: leukemia, lymphoma and myeloma. The term leukemia comes from the Greek white (leukos) and blood (haima). It generally refers to the abnormal production of immature blood cells, which cannot further differentiate into other mature blood cells, and it can be found in patients' blood and bone marrow. For leukemia patients, there are large amounts of immature blood cells circulating throughout the body which impair normal functions, including normal blood cell production and immune system activation. Based on the origin of the cell type affected, leukemia can be categorized into two main branches, myeloid and lymphoid.

\section{Chapter 1-3: Oncogenes}

In the past, all human cancer development was believed to be triggered by oncogenes, which were considered to originate from external factors such as retroviruses inserting fragments of viral DNA into the human body. This is partly due to the results of the experimental work conducted by Peyton Rous, which found out that cancer would present in healthy chickens if they were injected cellfree extract from chicken tumor cells. It was later found out that the extract encompassed the Rous sarcoma retrovirus (RSV), which was responsible for the development of sarcomas in chickens (Rous, 1911). However, the actual virus was not the transmissible agent, instead $v$-SRC, which contained in the RSV, is responsible for the tumor development and associated with nucleotide 
sequences in the DNA of healthy chickens. This hypothesis was confirmed by Stehelin et al. (Stehelin, 1976) as he identified the first oncogene SRC. Since then, researchers found that oncogenes are possessed by many organisms, and those genes are mainly responsible for the regulation of cell development and division. Oncogenes can be activated by genetic abnormalities, including insertion of new enhancers or promoter regions to a gene region (e.g. in Burkitt's lymphoma, the juxtaposition of $M Y C$ to the human immunoglobulin heavy chain enhancer regions occurs) (Joos, 1992). Also, translocations give rise to new gene fusions, such as BCR-ABL (Salesse, 2002) and EWS-ERG (Kaneko, 1997). Furthermore, gene amplification, such as MYCN in neuroblastoma (Yoshimoto, 1999), or activating mutations in RAF or RAS genes may also stimulate oncogenes (Downward, 2003). The activation of oncogenes usually promotes abnormal cell growth and inhibits regulated apoptosis, which results in cancer.

\section{Chapter 1-4: Tumor suppressor genes}

The notion of tumor suppressor genes was first outlined by Knudson (Knudson, 1971) in retinoblastoma. Retinoblastoma can be either unilateral or bilateral. In Knudson's hereditary retinoblastoma study, he collected data for retinoblastoma occurring in both unilateral and bilateral cases and found that only one somatic mutation was necessary in the case of bilateral retinoblastoma, as a germline mutation was already present in the cells, while two somatic mutations were necessary for unilateral retinoblastoma to occur. These observations later 
became the basis for the well-known 'two-hit hypothesis'. Later, Friend et al. (Friend, 1986) validated the hypothesis by discovering recessive mutations in both alleles of the Rb1 gene, which was the first tumor suppressor gene that was successfully replicated.

However, there are conditions which are exceptions to the 'two-hit' hypothesis. For example, the hereditary Li-Fraumeni syndrome which is associated with increased risk of breast, lung, colorectum and brain tumors, is an autosomal dominant condition which only needs one allele of TP53 to be mutated to have disease phenotype. The p53 protein is considered as an important protein not only because it coordinates responses throughout the cell cycle and mechanisms of DNA repair, but it also determines both cell development and apoptosis (Finlay, 1989) (Knudson, 2001). Inactivation of both Rb1 and TP53 pathways are directly correlated to the occurrence of most of the human cancers. Other than $R b 1$ and TP53, there are numerous tumor suppressor genes in humans, shown in Table 1 (Cooper, 2000).

\section{Table 1: Tumor suppressor genes list}

\begin{tabular}{|l|l|}
\hline Gene & Type of cancer \\
\hline$A P C$ & Colon/rectum carcinoma \\
\hline$B R C A 1$ & Breast and ovarian carcinomas \\
\hline$B R C A 2$ & Breast carcinoma \\
\hline$D P C 4$ & Pancreatic carcinoma \\
\hline INK4 & $\begin{array}{l}\text { Melanoma, lung carcinoma, brain tumors, leukemias, } \\
\text { lymphomas }\end{array}$ \\
\hline MADR2 & Colon/rectum carcinoma \\
\hline NF1 & Neurofibrosarcoma \\
\hline NF2 & Meningioma \\
\hline
\end{tabular}




\begin{tabular}{|l|l|}
\hline$p 53$ & $\begin{array}{l}\text { Brain tumors; breast, colon/rectum, esophageal, liver, and } \\
\text { lung carcinomas; sarcomas; leukemias and lymphomas }\end{array}$ \\
\hline PTC & Basal cell carcinoma \\
\hline PTEN & $\begin{array}{l}\text { Brain tumors; melanoma; prostate, endometrial, kidney, and } \\
\text { lung carcinomas }\end{array}$ \\
\hline$R b$ & $\begin{array}{l}\text { Retinoblastoma; sarcomas; bladder, breast, and lung } \\
\text { carcinomas }\end{array}$ \\
\hline$V H L$ & Renal cell carcinoma \\
\hline WT1 & Wilms' tumor \\
\hline
\end{tabular}

\section{Chapter 1-5: The two-hit model applies to leukaemogenesis}

The multiplication, hematopoietic differentiation and death of acute myeloid leukemia (AML) stem cells are aberrant due to genomic damage. It is possible that gene rearrangements and mutations can be beneficial to cell proliferation, differentiation and/or survival, and cells which possess these mutations can have advantages when compared with other cells (Fröhling, 2005) (Kelly, 2002). Activation mutations in cell-surface receptors like RAS or tyrosine kinases like FLT3 can enhance AML stem cell clonal expansion (Fröhling, 2005) (MoreauGachelin, 2006) (Kosmider, 2006). Hematopoietic differentiation is also disrupted by core-binding-factor gene rearrangements caused by $t(8 ; 21)$, inv (16), or $\mathrm{t}(16 ; 16)$, or by the $P M L-R A R A$ and $M L L$ gene rearrangements (Fröhling, 2005) (Moreau-Gachelin, 2006) (Kosmider, 2006). The two-hit model may provide reasonable explanations for the observation of FLT3-ITDS cytogenetic aberrations in AML patients who have $\mathrm{t}(15 ; 17)$. (Thiede, 2002) (Schnittger, 2002) (Schnittger, 2011), as well as for the occurrence of $t(8 ; 21)$ and 
inv (16) AML, both of which are frequently associated with KIT mutations (Dash, 2001) (Care, 2003) (Park, 2011).

Table 2: Common cytogenetic abnormalities in AML

\begin{tabular}{|l|}
\hline Common Abnormality \\
\hline$t(8 ; 21)(q 22 ; q 22)$ \\
\hline$t(15 ; 17)(q 24.1 ; q 21)$ \\
\hline inv $(16) / t(16 ; 16)$ \\
$(p 13.1 ; q 22)$ \\
\hline$t(6 ; 9)(p 23 ; q 34)$ \\
\hline$t(9 ; 22)(q 34 ; q 11.2)$ \\
\hline inv (3) (q21.3;q26.2) \\
\hline $11 q 23$ rearrangement \\
\hline+8 \\
\hline $5 q-/-5$ \\
\hline $7 q-/-7$ \\
\hline $20 q-$ \\
\hline
\end{tabular}

Chapter 1-6: The World Health Organization (WHO) classification of AML In 1999, The World Health organization created a classification system for AML which focuses on the immunological, cytogenetic, morphological and clinical characteristics of the disease (Harris, 1999). The FAB system was developed and published in the revised (2008) World Health Organization classification system, as the organization highlights that the field of hemato-oncology is changing towards a more comprehensive system (Vardiman, 2009). According to the WHO classification, AMLs are grouped into four categories: (i) AML with recurrent genetic abnormalities; (ii) AML with multilineage dysplasia; (iii) therapy-related disorders; therapy-related AML (t-AML) and therapy-related myelodysplastic syndromes (t-MDS); and (iv) AML not otherwise categorized. 
The WHO classification system identified 108 new diagnostic entities in hematopathology, involving 50 new or provisional leukaemia entries (Betz, 2010).

\section{Chapter 1-7: Somatic mutations in myeloid malignancies}

In a previous study, Grimwade et al. noted that there were approximately half of the AML patients will have abnormal cytogenetics karyotypes which can observed on standard karyotype analysis (Grimwade, 2010). Cytogenetic observations which are considered as favorable risk include $t(15 ; 17)$ (q24.1;q21), PML-RARA; t (8;21) (q22;q22), RUNX1-RUNX1T1; and inv (16)/t $(16 ; 16)(p 13.1 ; q 22)$ or $t(16 ; 16) \quad(p 13.1 ; q 22), C B F B-M Y H 1$, and those patients usually have a good response to chemotherapy. In contrast, patients with unfavorable cytogenetics observations, such as complex karyotype, -5 , del (5q), -7 , and abnormalities of $3 q$, usually have a less favorable outcome and survival. Treatments for those patients often require allogeneic stem cell transplant in order to prolong survival (Grimwade, 2016) (He, 2015).

Table 3: Genes which are recurrently somatically mutated in myeloid malignancies grouped by their functional class

\begin{tabular}{|l|l|}
\hline Functional class & $\begin{array}{l}\text { Specific example } \\
\text { mutations }\end{array}$ \\
\hline $\begin{array}{l}\text { Signaling and } \\
\text { kinase pathway }\end{array}$ & $\begin{array}{l}F L T 3, K R A S, N R A S, K I T, \\
\text { PTPN11, and NF1 }\end{array}$ \\
\hline
\end{tabular}




\begin{tabular}{|l|l|}
\hline $\begin{array}{l}\text { Epigenetic } \\
\text { modifiers (DNA } \\
\text { methylation and } \\
\text { chromatin } \\
\text { modification) }\end{array}$ & $\begin{array}{l}\text { DNMT3A, IDH1, IDH2, } \\
\text { TET2, ASXL1, } \\
\text { EZH2, and MLL/KMT2A }\end{array}$ \\
\hline $\begin{array}{l}\text { Transcription } \\
\text { factors }\end{array}$ & $\begin{array}{l}\text { CEBPA, } \\
\text { RUNX1, and GATA2 }\end{array}$ \\
\hline $\begin{array}{l}\text { Tumor } \\
\text { suppressors }\end{array}$ & TP53 \\
\hline $\begin{array}{l}\text { Spliceosome } \\
\text { complex }\end{array}$ & $\begin{array}{l}\text { SRSF2, U2AF1, } \\
\text { SF3B1, and ZRSR2 }\end{array}$ \\
\hline Cohesin complex & $\begin{array}{l}\text { RAD21, STAG1, STAG2, } \\
\text { SMC1A, and SMC3 }\end{array}$ \\
\hline
\end{tabular}

In recent years, researchers have gained plethora of knowledge about the genetic basis of AML, thanks to the advancement of diagnosing technology such as fluorescence in situ hybridization (FISH), genome array, Sanger sequencing, whole exome sequencing (WES), whole genome sequencing (WGS), and RNA sequencing. To date, there are $\sim 52$ genes that are shown to have associations with myeloid malignancy (Grimwade, 2016). Genes that are mostly mutated in myeloid malignancies can be grouped into at least six groups: 1 . Signaling and kinase pathway mutations; 2. Epigenetic modifiers; 3. Transcription factors; 4. Tumor suppressors; 5. Spliceosome complex and 6. Cohesin complex (DiNardo, 2016). Myeloid malignancies are known to frequently have mutations in specific genes, such as RUNX1 and TET2 (Harada, 2015) (Figueroa, 2010). FLT3 mutations are known to cause de novo AML, and TET2 mutations are known to have association with MDS. 
Genes that are important for malignancy onset and progression tend to be those that acquire mutations somatically, or that are subject to somatic duplication or deletion events. As previously mentioned, germline variants can interact with somatic mutations, which is similar to Knudson's "two-hit" hypothesis. For example, RUNX1 is frequently mutated somatically (Harada, 2004) in myeloid malignancy patients, and also harbors known germline predisposition variants (Walker, 2002). Similarly, CEBPA can carry germline and somatic mutations, often in the same acute myeloid leukemia (AML) patient (Pabst and Mueller 2009). Makishima et al. recently reported (Makishima, Yoshida et al. 2013) somatic SETBP1 mutations in secondary AML that affect precisely the same amino acid as do known germline mutations causing Schinzel-Gideon syndrome, a congenital disease carrying an elevated likelihood of tumors. Another example of germline-somatic convergence is the presence of PRPF8 somatic mutations and hemizygous deletions in MDS and related conditions (Kurtovic-Kozaric, Przychodzen et al. 2014). PRPF8 is the gene in which germline mutations cause retinitis pigmentosa. More recently, Polprasert et al. have observed both somatic mutations and germline frameshift mutations in DDX41 in MDS patients (Polprasert, 2015).

\section{Chapter 1-8: Myeloid Malignancies: Knowns and Unknowns}

Myeloid malignancies include leukemias or preleukemias that originate from the myeloid lineage in the hematopoietic stem cell differentiation pathway. They are clonal diseases, and their causes include genetic and epigenetic alterations that 
disturb key processes of cells such as growth, regeneration and death (Murati, 2012). Diseases included in myeloid malignancies are primary and secondary acute myeloid leukemia (pAML and sAML), myelodysplastic syndrome (MDS), myeloproliferative neoplasms (MPNs), and myelodysplastic syndrome/myeloproliferative neoplasms (MDS/MPN).

The completion of the human genome project (Lander, 2001) (Venter, 2001) opened a new era of genetic studies. It allows scientists to conduct genome wide research projects and can change clinicians' therapeutic method from chemotherapy or radiotherapy to personalized medicine. Furthermore, with the revolution in next-generation sequencing and the sequencing of the human genome, the approach has been to sequence cancer genomes to find mutations across the scale spectrum, from large translocations to base-pair changes. Large-scale projects such as the Cancer Genome Atlas (TCGA) (Cancer Genome Atlas Research, 2013), The Therapeutically Applicable Research to Generate Effective Treatments (TARGET) (Farrar, 2016), the International Cancer Genome Consortium (ICGC) (International Cancer Genome, 2010), and others have created numerous databases with a plethora of sequencing data available to researchers. Using those data, researchers have been able to find new insights in numerous types of cancer such as ovarian (Bolton, 2010), breast (Purrington, 2014), and colon (Won, 2012) and therefore our knowledge about cancer has been expanded at a rapid rate. 
Instead of generalizing about all patients based on the origin of a tumor, researchers are now focusing on the genomic profile of individual tumors. This has led to changes in drug development for cancer from targeting specific cancer types to targeting specific changes or mutations that cancer cells have. One successful example is Gleevec which specifically targets chronic myelogenous leukemia $(\mathrm{CML})$ which is caused by the $B C R-A B L$ translocation. The estimated overall survival rate for patients is $89 \%$, with a relapse rate of only about $17 \%$ in CML patients after Gleevec treatment (Druker, 2006). Therefore, exploring the opportunity of precision medicine can be beneficial to patients as this can provide the most effective treatment based on individual differences. It can also increase our understanding of cancer.

While there is extensive research on somatic mutations, we have little information about germline variants in myeloid malignancy. Genome wide association studies (GWAS) have been performed to find associations between particular SNPs and other disease phenotypes. Many GWAS have been successful in certain diseases, such as in cystic fibrosis (CF) (Wright, 2011), age-related macular degeneration (AMD) (Naj, 2013) (Arakawa, 2011) (Klein, 2005), diabetes (Blackman, 2013) (Ma, 2014) (Hanson, 2014), and multiple sclerosis (Leone, 2013) (Martinelli-Boneschi, 2012). Although GWAS find a good number of association in many human diseases, the percent trait variance explained by GWAS signals is not enough to explain all the heritability. This is called "missing heritability". In the case of myeloid malignancy, there are only a 
few GWAS publications on acute myeloid leukemia or myelodysplastic syndrome. There was almost no association found between common SNPs and disease phenotype, and the heritable component for myeloid malignancy remains undiscovered.

\section{Chapter 1-9: The importance of rare variants}

Exome-wide and genome-wide sequencing studies has given researchers insight to understand the effects of rare variants. By understanding rare variants, researchers may be able to explain some of the missing heritability of many human traits (Agarwala, 2013). Also, differential selective pressures acting on variants across the allele frequency spectrum tend to be skewed in favor of rare variants for traits most strongly influenced by natural selection, compared with quantitative phenotypes or late-onset diseases (Park, 2011). By discovering the associations driven by low-frequency and rare variants through genome sequencing and large-scale imputation efforts, researchers can make continuous improvements on trait heritability across the frequency spectrum (Kryukov, 2009).

It is also worth noting that rare variants in or near gene targets have a larger effect on phenotype (Consortium, 2015) (Bouatia-Naji, 2009). Understanding these variants through the next generation sequencing of protein-coding regions will bring advancements in annotating genetic associations with complex diseases and describing the functional consequences of human sequence variation. 


\section{Chapter 1-10: Difficulties in conducting GWAS in myeloid malignancies}

Myeloid malignancies are rare diseases, as their incident rate is less than 15 per 100,000 persons. This creates a big challenge for researchers who want to conduct genome wide association studies on these diseases because small numbers of patients translates into insufficient statistical power. Nevertheless, several studies such as TCGA, TARGET, and St Jude's Pediatric Cancer Genome Project (PCGP) are trying to gather many patients with myeloid malignancies and set up large databases in order to ease the sample size problem. Those studies also provide sequencing data and results for researchers to download and reanalyze, and hence facilitate the advancement on myeloid malignancy research.

Apart from sample size, cofounding effects caused by genetic admixture is another problem in many GWAS. Genetic admixture occurs when individuals from different populations interbreed. As a result, the genomes of the individuals in an admixed population become a mixture, in which chromosomal segments can be coming from different ancestral populations. Thus, each chromosomal segments have their own identity, or "local ancestry", and an individual would have proportion of different ancestry in his/her genome. (Liu, 2013).

In addition, cross contamination within individuals is also a source of false positive in sequencing analysis. As cancer (or pre-cancer) cells have the ability to invade healthy compartments, acquiring pure normal tissue from patients can be a problem for all cancer association studies (Cancer Genome Atlas 
Research, 2013) (Taylor-Weiner, 2018) (Stieglitz, 2015) (Wei, 2016). Therefore, DNA which originates from normal healthy samples may sometimes contain tumor cells and this type of contamination has been reported in various diseases such as leukemias (Stieglitz, 2015) (Welch, 2012) (Landau, 2015), and breast, bladder, and gastric cancers (Deng, 1996) (Försti, 2001) (Leung, 2001), among others. This type of contamination creates false positives, which decrease the accuracy for germline variant detection through next generation sequencing methods. Therefore, avoiding cross contamination within individuals is a crucial quality control step for all association studies in cancer (Taylor-Weiner, 2018).

\section{Chapter 1-11: Rationale for this study}

For nearly 15 years, genome wide association studies (GWAS) have been conducted to find associations between inherited polymorphisms and disease phenotypes. Although the approach has seen success in many human malignancies, there has been no published GWAS of acute myeloid leukemia (AML) or myelodysplastic syndrome (MDS). This is despite the fact that several studies (Richkind, Loew et al. 1998, Lichtenstein, Holm et al. 2000, Kerber and O'Brien 2005, Goldin, Kristinsson et al. 2012) show a heritable component in myeloid malignancy. Indeed, first-degree relatives of MDS carriers have double the likelihood of acquiring the disease (Strom, Gu et al. 2005), and leukemias in general affect children at a higher rate than do any other cancer. However, AML (excepting pediatric forms) and MDS are typically very late age-of-onset diseases, which complicates efforts to identify susceptibility variants. Case-control studies 
may be hampered by a lack of certainty that the controls will not eventually acquire the disease.

The limited number of variants that have been implicated in myeloid malignancy susceptibility are mostly rare and have largely been identified in families (Churpek, Pyrtel et al. 2015, Godley and Shimamura 2017). Newfound predisposition alleles are also likely to be rare, given the dearth of published common susceptibility alleles and the nature of known susceptibility variants. Identifying new variants will enable surveillance and intervention for carriers and will inform appropriate choices for hematopoietic stem cell transplantation. Causal inherited alleles also serve as potential targets for therapeutics. There have been recent successes in this regard in solid tumors. Carriers of germline BRCA1/2 mutations were shown to be responsive to poly (ADP-ribose) polymerase (PARP) inhibitors for multiple cancer types (Kaufman, Shapira-Frommer et al. 2015), and the PARP inhibitor olaparib has achieved regulatory approval for ovarian cancer patients. Furthermore, potentially targetable inherited variants in DNA-repair genes were shown to be frequent in men with metastatic prostate cancer (Pritchard, Mateo et al. 2016).

In this study, we analyze whole-exome sequence data from the germlines of 690 patients with myeloid malignancies. Much of this data has been obtained from studies that focused exclusively on the somatic mutational landscape. These studies used the germline exomes only to distinguish somatic mutations from inherited variants. We therefore exploit data that is largely discarded and was not mined for clues to inherited contributors to disease. We assess the presence and 
impact of rare variants in genes with previously implicated susceptibility alleles, as well as in genes with known or putative roles in myeloid neoplasms or cancer in general. In an effort to identify new germline contributors, we also focus particularly on genes that are known to demonstrate an autosomal recessive inheritance pattern, including Fanconi anemia genes. The hypothesis here is that germline contributors may be identified by their appearing frequently paired, in the same patient, in autosomal recessive genes, impacting both parental homologs. Synergies both among rare variants and with somatic mutations are also investigated, under the hypothesis that a wild-type copy paired with a germlinedisrupted copy may itself be disrupted somatically. 


\section{Chapter 2: Patient collections, variant calling and description of variants}

\section{Chapter 2-1: Germline whole exome sequencing and ancillary data collection}

We obtained whole-exome germline bam files from myeloid malignancy patients from the Cleveland Clinic and the Josep Carreras Leukaemia Research Institute in Barcelona, Spain. We also downloaded whole exome bam files from online sources (Table 5), yielding a total of 690 cases. For all cases, DNA was collected from either buccal mucosa or paired T-lymphocytes (CD3 ${ }^{+}$cells). We obtained available somatic mutation, cytogenetic, and clinical information for the patient datasets.

Table 4: Patient sample sources

\begin{tabular}{|l|l|l|l|l|}
\hline \multicolumn{3}{|c|}{$\begin{array}{l}\text { Number } \\
\text { of patient } \\
\text { samples }\end{array}$} & Diagnoses & $\begin{array}{l}\text { Publication } \\
\text { (PubMed }\end{array}$ \\
ID)
\end{tabular}




\begin{tabular}{|c|c|c|c|c|}
\hline Multiple origins & phs001027.v1.p1 (dbGaP) & 47 & pAML, sAML & 27322744 \\
\hline $\begin{array}{l}\text { Gustave } \\
\text { Roussy Cancer } \\
\text { Center }\end{array}$ & EGAD00001001853 & 39 & MDS/MPN & 26908133 \\
\hline $\begin{array}{l}\text { International } \\
\text { Cancer } \\
\text { Genome } \\
\text { Consortium } \\
\text { (ICGC) }\end{array}$ & EGAD00001000123 & 50 & MPN & NA \\
\hline ICGC & EGAD00001000106 & 27 & MPN & NA \\
\hline ICGC & EGAD00001000117 & 39 & MDS & 25957392 \\
\hline $\begin{array}{l}\text { Institutions } \\
\text { throughout } \\
\text { Japan }\end{array}$ & EGAS00001000521 & 6 & MDS/MPN & 23832011 \\
\hline $\begin{array}{l}\text { Therapeutically } \\
\text { Applicable } \\
\text { Research To } \\
\text { Generate } \\
\text { Effective } \\
\text { Treatments } \\
\text { (TARGET) }\end{array}$ & GDC & 22 & pAML & 26941285 \\
\hline $\begin{array}{l}\text { The Cancer } \\
\text { Genome Atlas } \\
\text { (TCGA) }\end{array}$ & GDC & 142 & pAML & 23634996 \\
\hline
\end{tabular}

Table 5: Patient summary

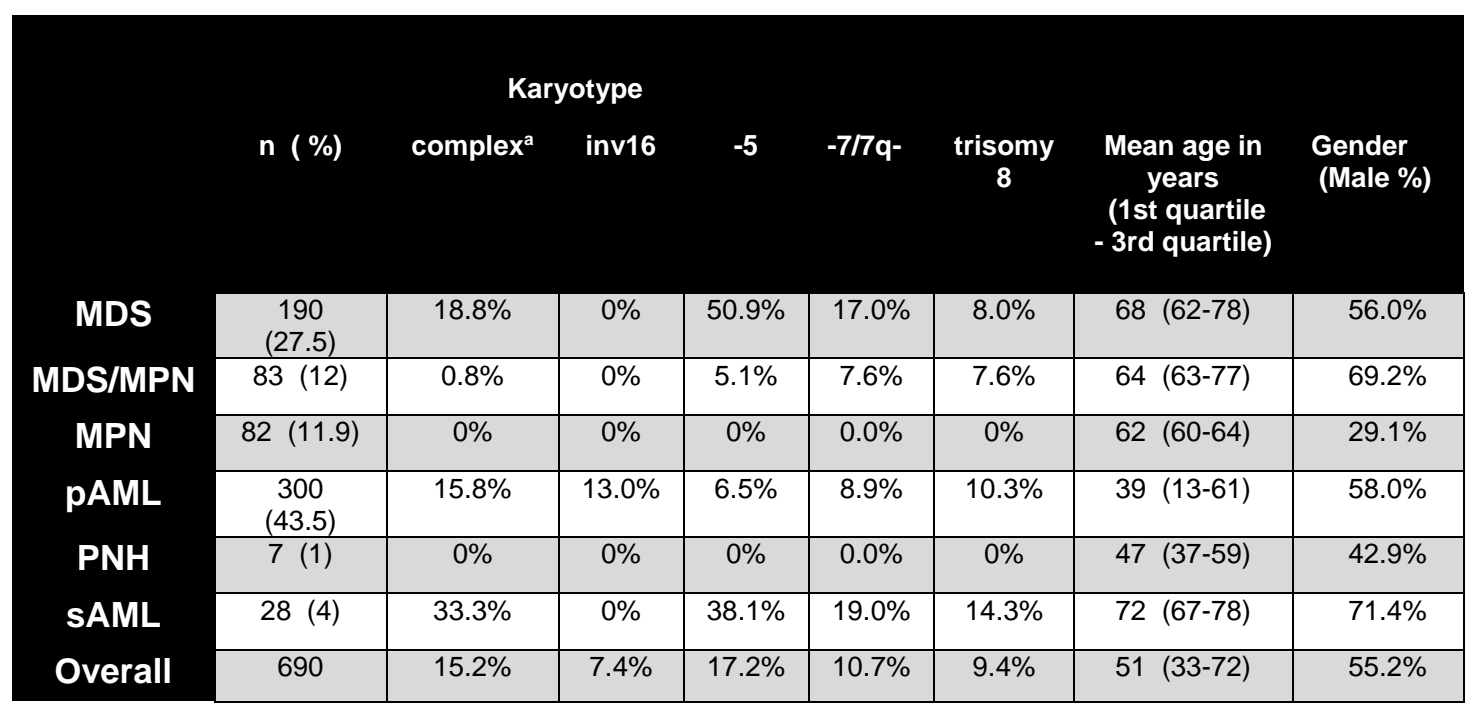

a Three or more cytogenetic lesions

Table 6: Patient cohort 


\begin{tabular}{|c|c|c|c|c|c|c|}
\hline Patient ID & $\begin{array}{l}\text { Age at } \\
\text { diagnosis }\end{array}$ & Gender & Diagnosis & $\frac{\frac{\underline{\text { Status }}}{(0=\text { dead }}}{\frac{1=\text { alive }}{\text { NA =not }}}$ & $\frac{\frac{\text { Overall }}{\text { survival }}}{\text { (mos) }}$ & Karyotype \\
\hline AML_074 & 71 & $M$ & $\mathrm{pAML}$ & NA & NA & $\begin{array}{c}49, X Y,+13,+1 \\
3,+19[21]\end{array}$ \\
\hline AML_075 & 49 & $F$ & pAML & $\mathrm{NA}$ & $\mathrm{NA}$ & $46, X X[22]$ \\
\hline AML_078 & 31 & $F$ & $\mathrm{pAML}$ & NA & NA & $\begin{array}{c}46, X X, \text { inv } \\
(16) \\
(p 13 q 22)[8] / 4 \\
7, X X,+8, i n v \\
(16) \\
\text { (p13q22)[12] }\end{array}$ \\
\hline AML_079 & 54 & $F$ & pAML & NA & NA & $\begin{array}{c}47, X X,+21 \\
{[21] / 46, X X} \\
{[7]}\end{array}$ \\
\hline AML_080 & 33 & $M$ & $\mathrm{pAML}$ & NA & NA & $\begin{array}{c}47, X Y,+11 \\
{[43] / 46, X Y} \\
{[5]}\end{array}$ \\
\hline AML_081 & 43 & $\mathrm{M}$ & $\mathrm{pAML}$ & NA & NA & $\begin{array}{c}47, X Y,+11 \\
{[14] / 46, X Y} \\
{[13]}\end{array}$ \\
\hline AML_082 & 37 & $\mathrm{M}$ & pAML & NA & NA & $\begin{array}{c}46, X Y, \text { del } \\
(11)(q 14 \\
q 25)[3] / 46 \\
X Y[60]\end{array}$ \\
\hline AML_083 & 62 & M & pAML & NA & NA & $46, X Y[68]$ \\
\hline AML_084 & 37 & $M$ & $\mathrm{pAML}$ & NA & NA & $\begin{array}{c}47, X Y,+13[11 \\
] / 46, X Y[28]\end{array}$ \\
\hline AML_086 & 30 & $\mathrm{M}$ & pAML & $\mathrm{NA}$ & NA & $46, X Y[26]$ \\
\hline AML_088 & 30 & $F$ & pAML & NA & NA & $46, X X[21]$ \\
\hline AML_089 & 60 & $F$ & pAML & NA & NA & $46, \mathrm{XX}[24]$ \\
\hline AML_090 & 42 & M & pAML & NA & NA & $46, X Y[20]$ \\
\hline AML_091 & 45 & M & pAML & NA & NA & $\begin{array}{c}46, X Y, \text { add } \\
(7) \\
(q 22)[4] / 46, X \\
Y[21] \\
\end{array}$ \\
\hline AML_092 & 41 & M & pAML & NA & NA & $\begin{array}{c}46, X Y, \text { inv } \\
(16) \\
\text { (p13q22)[21] }\end{array}$ \\
\hline AML_093 & 53 & M & pAML & NA & NA & $46, X Y[23]$ \\
\hline AML_094 & 50 & $F$ & pAML & NA & NA & $46, \mathrm{XX}[24]$ \\
\hline AML_095 & 49 & $F$ & $\mathrm{pAML}$ & NA & NA & $46, X X[20]$ \\
\hline AML_096 & 43 & $F$ & pAML & NA & NA & $\begin{array}{c}46, X X, \text { del } \\
(11) \\
(q 11 q 14), \text { inv } \\
(16) \\
\text { (p13q22)[16] }\end{array}$ \\
\hline
\end{tabular}




\begin{tabular}{|c|c|c|c|c|c|c|}
\hline AML_097 & 72 & $M$ & pAML & NA & $\mathrm{NA}$ & $\begin{array}{c}46, X Y, t \\
(3 ; 21) \\
(q 26 ; q 22), \text { del } \\
(12) \\
\text { (p12p13)[20] }\end{array}$ \\
\hline AML_098 & 67 & $F$ & pAML & NA & NA & $46, \mathrm{XX}[25]$ \\
\hline AML_099 & 61 & $M$ & pAML & NA & NA & $\begin{array}{c}\operatorname{mos} 47, X X Y[2 \\
7] / 46, X Y[4]\end{array}$ \\
\hline AML_100 & 65 & $\mathrm{M}$ & pAML & NA & NA & $\begin{array}{c}56 \sim 58, X Y,+X \\
\text { der (5)del (5) } \\
\text { (p1?2p1?4)d } \\
\text { el (5) } \\
\text { (q1?3q15),+8 } \\
,+10,+11,+11 \\
,+13,+19,+21 \\
,+22,+ \text { mar1, }+ \\
\text { mar2[8]/56 5 } \\
\text { 8,idem,add } \\
\text { (2) } \\
\text { (q3?6)[16]/46 } \\
\text {,XY[30] }\end{array}$ \\
\hline AML_101 & 63 & $M$ & pAML & NA & NA & $\begin{array}{c}47, X Y,+8[1] / 4 \\
6, X Y[34]\end{array}$ \\
\hline AML_102 & 62 & $M$ & $\mathrm{pAML}$ & NA & NA & 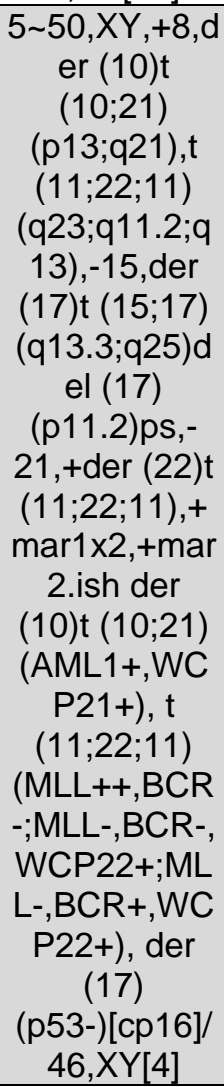 \\
\hline AML_103 & 50 & $\mathrm{~F}$ & $\mathrm{pAML}$ & NA & $\mathrm{NA}$ & $46, X X$ \\
\hline
\end{tabular}




\begin{tabular}{|c|c|c|c|c|c|c|}
\hline AML_104 & 67 & $\mathrm{~F}$ & pAML & NA & NA & $\begin{array}{c}46, X X, t \\
(2 ; 11) \\
\text { (p25;q13),- } \\
9, \text { del (9) } \\
\text { (p13),add } \\
\text { (13) (p11.2),- } \\
\text { 17,+mar1,+m } \\
\text { ar2[cp13]/46, } \\
\text { XX[2] }\end{array}$ \\
\hline AML_105 & 54 & $\mathrm{M}$ & pAML & NA & NA & $46, X Y$ \\
\hline AML_106 & 61 & $M$ & pAML & NA & NA & $46, X Y$ \\
\hline AML_108 & 67 & $\mathrm{~F}$ & pAML & NA & NA & $\mathrm{NA}$ \\
\hline AML_109 & 18 & $M$ & pAML & NA & NA & $46, X Y$ \\
\hline AML_110 & 49 & $\mathrm{~F}$ & pAML & NA & $\overline{N A}$ & $46, X X$ \\
\hline AML_111 & 28 & $\mathrm{~F}$ & $\mathrm{pAML}$ & NA & $\mathrm{NA}$ & $46, X X$ \\
\hline AML_113 & 23 & $\mathrm{~F}$ & pAML & NA & NA & $46, X X$ \\
\hline AML_114 & 56 & $M$ & pAML & NA & NA & $46, X Y$ \\
\hline AML_115 & 42 & $\mathrm{~F}$ & pAML & NA & NA & $\begin{array}{c}46, \mathrm{XX}, \mathrm{t}(5 ; 8) \\
\text { (q31.3;q21) }\end{array}$ \\
\hline AML_116 & 64 & $M$ & pAML & NA & NA & $\begin{array}{c}47, X Y,+4, \text { del } \\
(4) \\
(q 12 q 31)[7] / 4 \\
6, X Y[13]\end{array}$ \\
\hline AML_119 & 64 & $\mathrm{~F}$ & pAML & NA & NA & $46, \mathrm{XX}[12]$ \\
\hline AML_121 & 74 & $\mathrm{M}$ & pAML & NA & NA & $46, X Y[25]$ \\
\hline AML_123 & 57 & $\mathrm{M}$ & pAML & NA & NA & $46, X Y[18]$ \\
\hline AML_124 & 65 & $M$ & pAML & NA & NA & $\begin{array}{c}47, \mathrm{XY},+21[8] / \\
46, \mathrm{XY}[16] \\
\end{array}$ \\
\hline AML_126 & 60 & $M$ & pAML & NA & NA & $\begin{array}{c}46, X Y[2] \\
\text { POS FOR del } \\
5 q 3110 \text { IN } \\
200 \text { CELLS / } \\
\text { FISH FOR } \\
\text { CEP 7/del } \\
\text { 7q31 NEG IN } \\
200 \text { CELLS }\end{array}$ \\
\hline AML_127 & 66 & $M$ & pAML & NA & NA & unknown \\
\hline AML_128 & 49 & $M$ & pAML & NA & NA & $46, X Y[1]$ \\
\hline AML_133 & 57 & $\mathrm{~F}$ & pAML & NA & NA & $46, X X[27]$ \\
\hline AML_135 & 74 & $\mathrm{~F}$ & pAML & NA & NA & $\begin{array}{c}\text { monosomy } 7 \\
{[7 / 17]}\end{array}$ \\
\hline AML_139 & 55 & $\mathrm{~F}$ & pAML & NA & NA & $46, X X$ \\
\hline C_01 & 84 & $\mathrm{M}$ & sAML & 0 & $\begin{array}{c}15.3534 \\
2466\end{array}$ & $\begin{array}{c}46, X Y, \text { del } \\
(20) \\
(q 11.2)[12]\end{array}$ \\
\hline C_02 & 70 & $M$ & MDS/MPN & 1 & $\begin{array}{c}78.0821 \\
9178 \\
\end{array}$ & $\begin{array}{l}46, X Y, \operatorname{del}(2) \\
\text { (p22),inv (9) }\end{array}$ \\
\hline
\end{tabular}




\begin{tabular}{|c|c|c|c|c|c|c|}
\hline & & & & & & $\begin{array}{l}\text { (p12q13), del } \\
(20)(q 12)[4] .\end{array}$ \\
\hline C_03 & 62 & $\mathrm{~F}$ & MDS/MPN & 0 & $\begin{array}{c}29.6547 \\
9452\end{array}$ & $\begin{array}{c}46, X X, \text { del } \\
(11) \\
(q 23)[3] / 46, X \\
X[17]\end{array}$ \\
\hline C_04 & 68 & $M$ & MDS & 1 & $\begin{array}{c}60.9863 \\
0137\end{array}$ & $\begin{array}{c}46, X Y, \text { ins } \\
(1 ; 14) \\
\text { (p32;q24q32) } \\
{[19] / 46, X Y[1]}\end{array}$ \\
\hline C_05 & 78 & $M$ & sAML & 0 & $\begin{array}{c}39.0904 \\
1096\end{array}$ & $46, \mathrm{XY}[13]$ \\
\hline C_06 & 60 & $M$ & MDS/MPN & 0 & $\begin{array}{c}35.6054 \\
7945\end{array}$ & $\begin{array}{c}46, X Y \\
(6 / 2005)\end{array}$ \\
\hline C_07 & 65 & $M$ & MDS & 1 & $\begin{array}{c}23.9013 \\
6986\end{array}$ & $\begin{array}{c}48, X Y, \text { del }(5) \\
\text { (q12q33),- } \\
6, \text { del } \\
\text { (7)q11),+8,-- } \\
13, \mathrm{i}(17) \\
(\mathrm{q} 10),+3 \mathrm{mar}[ \\
41 / 46, \mathrm{XY}[3]\end{array}$ \\
\hline C_08 & 79 & $M$ & sAML & 0 & $\begin{array}{c}77.6219 \\
1781\end{array}$ & $\begin{array}{c}46, X Y, d e l \\
(12) \\
\text { (p11.2p13)[c } \\
\text { p15]/46,XY,t } \\
(4 ; 16) \\
\text { (q12;p13.3),d } \\
\text { el (12) } \\
\text { (p11.2p13)[5] }\end{array}$ \\
\hline C_09 & 69 & $M$ & sAML & 1 & 96 & $46, X Y[20]$ \\
\hline C_10 & 67 & $M$ & sAML & 0 & $\begin{array}{c}20.2520 \\
5479\end{array}$ & $\begin{array}{c}41-44, X Y, \text { del } \\
(1) \\
\text { (p21)[1],del } \\
(3) \\
\text { (q21q26.2)[2] } \\
\text {,inv (3) } \\
\text { (q21q26.2),a } \\
\text { dd (5) (q22),-- } \\
\text { 6,add (7) } \\
\text { (q22),-- } \\
\text { 10[19],add } \\
\text { (11) (q23)[3],- } \\
\text { 12,- } \\
\text { 17,+mar1[19] } \\
\text {,+mar2[18][c } \\
\text { p20] }\end{array}$ \\
\hline C_101 & 31 & $\mathrm{~F}$ & $\mathrm{PNH}$ & 0 & $\begin{array}{c}144.131 \\
5068\end{array}$ & $46, X X$ \\
\hline C_102 & 85 & $F$ & MDS & 0 & $\begin{array}{c}12.4602 \\
7397\end{array}$ & $46, X X$ \\
\hline C_103 & 20 & $M$ & PNH & 0 & $\begin{array}{c}77.3260 \\
274\end{array}$ & $\mathrm{NA}$ \\
\hline
\end{tabular}




\begin{tabular}{|c|c|c|c|c|c|c|}
\hline C_106 & 77 & $\mathrm{~F}$ & MDS & 0 & $\begin{array}{c}17.5232 \\
8767\end{array}$ & $\begin{array}{c}45, X X,-7, \text { del } \\
(20) \\
(q 11.2 q 13.3)[ \\
13] / 46, X X[4]\end{array}$ \\
\hline C_107 & 80 & $M$ & MDS & 0 & $\begin{array}{c}49.6109 \\
589\end{array}$ & $\begin{array}{c}47, X Y,+ \text { der } \\
(1 ; 19) \\
(q 10 ; p 10)[20]\end{array}$ \\
\hline C_108 & 67 & $\mathrm{~F}$ & MDS & 1 & $\begin{array}{c}37.8410 \\
9589\end{array}$ & $\begin{array}{c}\text { 45,XX,del (3) } \\
\text { (p14),-5,-7, } \\
\text { add (17) } \\
(\mathrm{p} 11.2),+22[4 \\
] / 46, \mathrm{XX}[\mathrm{cp} 17]\end{array}$ \\
\hline C_11 & 71 & $\mathrm{~F}$ & sAML & 0 & $\begin{array}{c}13.9068 \\
4932\end{array}$ & $\begin{array}{c}44-47, X X, \text { del } \\
(5) \\
\text { (q12q33)[11], } \\
-7[9], \text { del (7) } \\
\text { (q22)[2],del } \\
(7) \\
\text { (q22q34)x3[1 } \\
\text { ],-8,-12,-16,- } \\
16,-17,- \\
18[10],+21[8] \\
,+1- \\
4 r[12],+ \text { mar1, } \\
+ \text { mar2[11],+ } \\
\text { mar3[10],+m } \\
\text { ar4[10][cp12]/ } \\
46, X X[8]\end{array}$ \\
\hline C_113 & NA & $\mathrm{F}$ & MDS/MPN & NA & NA & NA \\
\hline C_115 & 64 & $\mathrm{~F}$ & MPN & 1 & $\begin{array}{c}3.48493 \\
1507\end{array}$ & $46, X Y$ \\
\hline C_116 & 65 & $\mathrm{~F}$ & MPN & 1 & $\begin{array}{c}44.9753 \\
4247\end{array}$ & $\begin{array}{c}47, \mathrm{XX},+9, \mathrm{der} \\
(15) \mathrm{t}(1 ; 15) \\
(\mathrm{q} 21 ; \mathrm{p} 11.2)[2 \\
] / 46, \mathrm{XX},[1] .\end{array}$ \\
\hline C_117 & 31 & $M$ & MDS & 0 & $\begin{array}{c}11.6383 \\
5616\end{array}$ & $\begin{array}{c}45, X Y,-7 \\
{[20]}\end{array}$ \\
\hline C_12 & 63 & $\mathrm{M}$ & MDS & 1 & $\begin{array}{c}29.5561 \\
6438\end{array}$ & $\begin{array}{c}44, X,-Y,-7, \text { del } \\
\text { (20) } \\
(q 11.2)[c p 19] \\
/ 46, X Y[1]\end{array}$ \\
\hline C_125 & 61 & $M$ & PNH & 0 & 81 & NA \\
\hline C_127 & 56 & $\mathrm{~F}$ & sAML & 0 & $\begin{array}{c}0.75616 \\
4384\end{array}$ & $\begin{array}{c}\text { 45,XX,del (5) } \\
\text { (q12q33),-- } \\
7,+8, \text { del }(16) \\
\text { (q22),der } \\
(20) t(17 ; 20) \\
(q 21 ; q 13.3)[1 \\
6] / 46, \text { idem,- } \\
13,- \\
\text { 15,+mar1[4]. }\end{array}$ \\
\hline
\end{tabular}




\begin{tabular}{|c|c|c|c|c|c|c|}
\hline C_128 & 75 & $\mathrm{~F}$ & MDS & 0 & $\begin{array}{c}81.4027 \\
3973\end{array}$ & $46, X X$, del5q \\
\hline C_129 & 79 & $\mathrm{~F}$ & MDS & 1 & 35 & $\begin{array}{c}\text { 45,XX,del (5) } \\
\text { (q15q33), del } \\
(7)(q 22),- \\
22[\mathrm{cp} 2] / 46, X \\
\text { X[18] }\end{array}$ \\
\hline C_13 & 78 & $F$ & MDS & 0 & $\begin{array}{c}31.5287 \\
6712\end{array}$ & $\begin{array}{c}\text { 44,XX,-4,del } \\
\text { (5) (q12q33),- } \\
17, \text { add (19) } \\
\text { (p13)[3]/45,sl, } \\
\text {-add (19) } \\
\text { (p13),+19,+m } \\
\text { ar1[7]/44,sl1,-- } \\
6,+ \text { inv }(6) \\
\text { (p23q25),--, } \\
\text { 10,+del (10) } \\
\text { (q25),- } \\
\text { 14,+add (14) } \\
\text { (q32),-15,- } \\
22,+ \text { der }(22) t \\
(15 ; 22) \\
\text { (q11.2;q13),+ } \\
\text { mar2[cp6]/46, } \\
\text { XX[4] }\end{array}$ \\
\hline C_130 & 64 & $M$ & MDS/MPN & 0 & $\begin{array}{c}75.4849 \\
3151 \\
\end{array}$ & $\begin{array}{c}46, \mathrm{XY}, \mathrm{del}(5) \\
(\mathrm{q} 12 \mathrm{q} 33)[6]\end{array}$ \\
\hline C_132 & 70 & $\mathrm{~F}$ & MDS & 1 & $\begin{array}{c}23.5726 \\
0274\end{array}$ & $\begin{array}{c}47, X X,+8[2] / 4 \\
7, \text { idem,del (5) } \\
(q 22 q 33)[2] / 4 \\
6, X X[17]\end{array}$ \\
\hline C_133 & 74 & $M$ & sAML & 0 & $\begin{array}{c}26.1698 \\
6301\end{array}$ & $\begin{array}{c}45, X Y, \text { del }(5) \\
\text { (q13q34),del } \\
\text { (7) (q22),+8,- } \\
17,- \\
\text { 18[cp11]/46, } \\
\text { XY[9] }\end{array}$ \\
\hline C_134 & 75 & $\mathrm{M}$ & MDS & 0 & $\begin{array}{c}2.13698 \\
6301\end{array}$ & $\begin{array}{c}46- \\
47, X Y, A D D \\
(3) \\
(Q 11.2), D E L \\
(5) \\
(Q 13 Q 33),+8 \\
,-15,-16,+1- \\
\text { 2MAR[CP20] }\end{array}$ \\
\hline C_136 & 51 & $M$ & sAML & 0 & $\begin{array}{c}5.72054 \\
7945\end{array}$ & $\begin{array}{c}47, X Y,+21[16 \\
] / 47, \text { idem,add } \\
(7) \\
\text { (q36)[3]/46,X } \\
\text { Y[1] }\end{array}$ \\
\hline C_138 & 73 & $M$ & sAML & 0 & $\begin{array}{c}2.79452 \\
0548\end{array}$ & $\begin{array}{c}\text { 45-46,XY,der } \\
(3 ; 16) \\
(q 10 ; q 10)[19]\end{array}$ \\
\hline
\end{tabular}




\begin{tabular}{|c|c|c|c|c|c|c|}
\hline & & & & & & $\begin{array}{c}\text {,del (5) } \\
\text { (q22q35)[19], } \\
\text { add (12) } \\
\text { (p11.2)[19],a } \\
\text { dd (12) } \\
\text { (q13)[19],del } \\
(14) \\
\text { (q24q32)[19], } \\
\text {-17[9],add } \\
\text { (17) (q12)[9],- } \\
\text { 19[3],+add } \\
\text { (21) } \\
\text { (p12)[9],+22[ } \\
\text { 10],+mar1[3][ } \\
\text { cp19]/46,XY[ } \\
\text { 1] }\end{array}$ \\
\hline C_139 & 56 & $M$ & MDS & 0 & $\begin{array}{c}5.78630 \\
137\end{array}$ & $\begin{array}{c}45, \mathrm{XY}, \text { del }(5) \\
(\mathrm{q} 13 \mathrm{q} 33),-7,- \\
17,- \\
18,+\operatorname{mar} 1,+\mathrm{m} \\
\text { ar2[20] }\end{array}$ \\
\hline C_14 & 70 & $\mathrm{M}$ & MDS/MPN & 0 & $\begin{array}{c}20.4164 \\
3836\end{array}$ & $46, X Y[c p 20]$ \\
\hline C_140 & 70 & $M$ & MDS & 0 & $\begin{array}{c}6.01643 \\
8356\end{array}$ & $\begin{array}{c}\text { 43-44,XY, del } \\
\text { (5) (q13q35),-- } \\
\text { 7,add (9) } \\
\text { (p24),psu dic } \\
\text { (17;8) } \\
\text { (p13;p23),-- } \\
\text { 18,psu dic } \\
\text { (19;14) } \\
\text { (q13.4;p13),+ } \\
\text { 1-2r[cp20] }\end{array}$ \\
\hline C_141 & 57 & $\mathrm{~F}$ & MDS & 0 & $\begin{array}{c}130.783 \\
5616\end{array}$ & $\begin{array}{c}46, X X, \text { del }(5) \\
\text { (q13q33)[19]/ } \\
46, X X[1]\end{array}$ \\
\hline C_142 & 80 & $M$ & sAML & 0 & $\begin{array}{c}7.23287 \\
6712\end{array}$ & $\begin{array}{c}46, X Y, \text { del }(5) \\
\text { (q15q31)[11]/ } \\
46, X Y[9] .\end{array}$ \\
\hline C_143 & 72 & $\mathrm{~F}$ & sAML & 0 & $\begin{array}{c}6.93698 \\
6301\end{array}$ & $\begin{array}{c}\text { 44,XX, add } \\
\text { (3) (p11),add } \\
\text { (3) (q29), del } \\
\text { (5) } \\
\text { (q13q31), add } \\
\text { (9) (q11), der } \\
\text { (11) (11pter- } \\
>11 \text { q1?3::hsr } \\
\text { :14q11.2- } \\
>14 q \text { ter),hsr } \\
\text { (11) (q23),-- } \\
\text { 14,add (14) } \\
\text { (p12),-- }\end{array}$ \\
\hline
\end{tabular}




\begin{tabular}{|c|c|c|c|c|c|c|}
\hline & & & & & & $\begin{array}{c}19[\mathrm{cp} 19] / 46 \\
\text { XX[1] }\end{array}$ \\
\hline C_145 & 55 & $\mathrm{~F}$ & pAML & 1 & $\begin{array}{c}36.7561 \\
6438\end{array}$ & $\begin{array}{c}46, X X, \text { del }(5) \\
\text { (q31q35)[13]/ } \\
46, X X[7]\end{array}$ \\
\hline C_15 & 75 & $\bar{M}$ & MPN & 0 & $\begin{array}{c}12.4602 \\
7397\end{array}$ & $\begin{array}{c}47, X Y,+13, i \\
(17) \\
(q 10)[19] / 48, i \\
\text { dem },+5[1]\end{array}$ \\
\hline C_16 & 79 & $\mathrm{~F}$ & MDS & 0 & $\begin{array}{c}25.9397 \\
2603\end{array}$ & $\begin{array}{c}46, X X, \text { del }(5) \\
\text { (q15q33)[2]/4 } \\
6, \text { idem,t } \\
(2 ; 12) \\
\text { (p13;p11.2)[7 }\end{array}$ \\
\hline C_18 & 75 & $\bar{M}$ & $\overline{\text { sAML }}$ & 0 & $\begin{array}{c}69.6986 \\
3014\end{array}$ & $\begin{array}{c}45, \mathrm{X},- \\
\mathrm{Y}[7] / 46, \mathrm{XY}[1 \\
3]\end{array}$ \\
\hline C_19 & 65 & $\bar{M}$ & MDS & 0 & $\begin{array}{c}15.9452 \\
0548\end{array}$ & $46, X Y[20]$ \\
\hline C_20 & 50 & $F$ & MDS & 1 & $\begin{array}{c}28.0767 \\
1233\end{array}$ & $\begin{array}{c}46, X X, \text { del (9) } \\
\text { (q22q34), add } \\
\text { (20) } \\
\text { (q11.2)[18]/4 } \\
6, \text { idem,del (7) } \\
\text { (q21q35)[1]/4 } \\
6, X X[1] \\
(4 / 5 / 11)\end{array}$ \\
\hline C_21 & 69 & $\bar{M}$ & MDS/MPN & 0 & $\begin{array}{c}19.7260 \\
274\end{array}$ & $46, X Y[20]$ \\
\hline C_23 & 53 & $\mathrm{~F}$ & MDS/MPN & 1 & $\begin{array}{c}105.008 \\
2192\end{array}$ & $46, X X[15]$ \\
\hline C_24 & 53 & $\bar{M}$ & MPN & 1 & $\begin{array}{c}28.6684 \\
9315\end{array}$ & $46, X Y[21]$ \\
\hline C_26 & 66 & $\bar{M}$ & MDS/MPN & 1 & $\begin{array}{c}35.4739 \\
726\end{array}$ & $46, X Y[20]$ \\
\hline C_27 & 69 & $\mathrm{~F}$ & MDS & 0 & $\begin{array}{c}3.35342 \\
4658\end{array}$ & $\begin{array}{c}\text { 43,XX,-3,del } \\
\text { (5) (q22q35),-- } \\
\text { 7,add (12) } \\
\text { (p13),-- } \\
\text { 16[4]/43- } \\
\text { 44,idem,-- } \\
\text { 2,add (18) } \\
\text { (p11.3),+mar } \\
\text { 1,+mar2[cp1 } \\
\text { 5]/ 46,XX[1] }\end{array}$ \\
\hline C_28 & 63 & M & MDS/MPN & 1 & $\begin{array}{c}55.1342 \\
4658\end{array}$ & $46, X Y[20]$ \\
\hline C_29 & 74 & $\bar{M}$ & MDS & 0 & $\begin{array}{c}30.7397 \\
2603\end{array}$ & $46, X Y[20]$ \\
\hline C_30 & 81 & $\mathrm{~F}$ & MDS & 0 & $\begin{array}{c}56.1205 \\
4795\end{array}$ & $46, X X[20]$ \\
\hline
\end{tabular}




\begin{tabular}{|c|c|c|c|c|c|c|}
\hline C_31 & 64 & $M$ & sAML & 0 & $\begin{array}{c}46.7506 \\
8493\end{array}$ & $46, X Y[20]$ \\
\hline C_32 & 78 & $M$ & MDS & 0 & $\begin{array}{c}3.22191 \\
7808\end{array}$ & $\begin{array}{c}46, \mathrm{XY}, \mathrm{del} \\
(20) \\
(\mathrm{q} 11.2)[17]\end{array}$ \\
\hline C_33 & 65 & $M$ & MDS & 0 & $\begin{array}{c}30.8054 \\
7945\end{array}$ & $\begin{array}{c}46, X Y, \text { del }(5) \\
(q 12 q 33), \text { del } \\
(13) \\
(q 12 q 14)[20]\end{array}$ \\
\hline C_34 & 83 & $\mathrm{M}$ & SAML & NA & NA & $46, X Y[20]$ \\
\hline C_35 & 42 & $F$ & PNH & 0 & $\begin{array}{c}138.969 \\
863\end{array}$ & NA \\
\hline C_36 & 72 & $F$ & $\mathrm{PNH}$ & 0 & $\begin{array}{c}524.646 \\
5753\end{array}$ & $46, X X[19]$ \\
\hline C_38 & 64 & $M$ & MDS/MPN & 1 & 10 & $46, X Y[20]$ \\
\hline C_39 & 69 & $M$ & MDS/MPN & 0 & $\begin{array}{c}27.2219 \\
1781 \\
\end{array}$ & $46, \mathrm{XY}[15]$ \\
\hline C_40 & 81 & $\mathrm{~F}$ & MDS & 1 & $\begin{array}{c}64.2082 \\
1918\end{array}$ & $\begin{array}{c}46, \mathrm{XX}, \mathrm{del} \\
(20) \\
(\mathrm{q} 11.2)[20]\end{array}$ \\
\hline C_41 & 76 & $F$ & MDS & 0 & $\begin{array}{c}38.0383 \\
5616\end{array}$ & $\begin{array}{c}49, X X,- \\
7,+8,+8,+9,+ \\
13[6] / 46, X X[1 \\
4]\end{array}$ \\
\hline C_42 & 60 & $F$ & MDS & NA & NA & $\begin{array}{c}45, \mathrm{XX},- \\
7[1] / 46, \mathrm{XX}, \mathrm{de} \\
\text { I (11) } \\
(\mathrm{q} 23)[\mathrm{cp} 2] / 47 \\
\text {,XX,+11[cp26 } \\
] / 46, \mathrm{XX}[1]\end{array}$ \\
\hline C_43 & 59 & $\mathrm{~F}$ & sAML & 0 & $\begin{array}{c}48.0986 \\
3014 \\
\end{array}$ & $46, X X[20]$ \\
\hline C_44 & 76 & $\mathrm{M}$ & MDS/MPN & 0 & $\begin{array}{c}15.3863 \\
0137\end{array}$ & $46, X Y[20]$ \\
\hline C_45 & 66 & $\mathrm{M}$ & MDS & 0 & $\begin{array}{c}46.9150 \\
6849\end{array}$ & $46, X Y[20]$ \\
\hline C_46 & 83 & $F$ & MDS & 0 & $\begin{array}{c}35.4739 \\
726\end{array}$ & $46, \mathrm{XX}[20]$. \\
\hline C_47 & 71 & $F$ & MDS/MPN & 0 & $\begin{array}{c}4.01095 \\
8904\end{array}$ & $\begin{array}{c}88- \\
89<4 n>, X X X \\
\text { X,ins }(4: ?) \\
(q 12 ; ?) \times 2,-5,- \\
5,-7,-7, \text { der } \\
(8) t(7 ; 8) \\
(q 11.2 ; q 24) x \\
2,-13,-13,- \\
19,-19,+3- \\
5 m a r[c p 5] / 46, \\
\text { XX[17]. } \\
\end{array}$ \\
\hline C_48 & 62 & $\mathrm{M}$ & MDS & 1 & 100.8 & $47, X Y,+Y[20]$ \\
\hline C_49 & 65 & $\mathrm{M}$ & MDS & 0 & $\begin{array}{c}41.5890 \\
411\end{array}$ & $46, X Y$ \\
\hline
\end{tabular}




\begin{tabular}{|c|c|c|c|c|c|c|}
\hline C_50 & 60 & $\mathrm{M}$ & MDS/MPN & 0 & $\begin{array}{c}35.1123 \\
2877\end{array}$ & $45, X,-Y[20]$ \\
\hline C_51 & 64 & $M$ & MDS & NA & 36 & $46, X Y[20]$. \\
\hline C_52 & 79 & $\mathrm{~F}$ & MDS & 1 & $\begin{array}{c}84.2958 \\
9041\end{array}$ & $\begin{array}{c}46, X X, \text { del } \\
(20) \\
(q 11.2 q 13.1)[ \\
\text { 18]/46,idem,t } \\
(1 ; 15) \\
\text { (p36.3;q11.2) } \\
{[2]}\end{array}$ \\
\hline C_53 & 75 & $\mathrm{M}$ & MDS/MPN & 0 & $\begin{array}{c}39.8136 \\
9863\end{array}$ & $46, X Y[20]$ \\
\hline C_54 & 65 & $\mathrm{~F}$ & MDS/MPN & 0 & $\begin{array}{c}31.7917 \\
8082\end{array}$ & $46, \mathrm{XX}[20]$ \\
\hline C_55 & 66 & $\mathrm{~F}$ & MDS & NA & NA & $\begin{array}{c}45, X,- \\
X[4] / 46, X X[1 \\
6]\end{array}$ \\
\hline C_56 & 78 & $\mathrm{~F}$ & MDS/MPN & 0 & $\begin{array}{c}17.2273 \\
9726\end{array}$ & NA \\
\hline C_57 & 67 & $M$ & MDS & $\mathrm{NA}$ & NA & $46, X Y[20]$ \\
\hline C_58 & 61 & $\mathrm{~F}$ & MDS & NA & $\mathrm{NA}$ & $46, X X$ \\
\hline C_59 & 77 & $\mathrm{M}$ & sAML & 0 & $\begin{array}{c}28.8328 \\
7671\end{array}$ & $\begin{array}{c}42, X Y, \text { add } \\
\text { (1) }(q 32), \text { del } \\
\text { (3) }(q 12), \text { add } \\
\text { (4) }(q 21), \text { del } \\
\text { (5) }\end{array}$ \\
\hline C_60 & 77 & $M$ & MDS & 0 & $\begin{array}{c}33.8958 \\
9041\end{array}$ & $46, X Y[16]$ \\
\hline C_61 & 72 & $\mathrm{M}$ & MDS/MPN & NA & NA & $46, X Y$ \\
\hline C_62 & 42 & $\mathrm{~F}$ & MDS & 1 & $\begin{array}{c}91.5945 \\
2055\end{array}$ & $\begin{array}{c}46 \mathrm{XX} \text { in } 19 \\
\text { metaphases } \\
\text { and } 45, \mathrm{X},-\mathrm{X} \\
\text { in one } \\
\text { metaphase }\end{array}$ \\
\hline C_64 & 66 & $M$ & MDS/MPN & 0 & $\begin{array}{c}11.4739 \\
726\end{array}$ & $46, X Y[20]$ \\
\hline C_65 & 41 & $F$ & MDS/MPN & 1 & $\begin{array}{c}19.3643 \\
8356\end{array}$ & $\begin{array}{c}46, X X, \text { del } \\
(13) \\
(q 12 q 22)[18] / \\
46, X X[2]\end{array}$ \\
\hline C_66 & 75 & $M$ & MDS/MPN & 1 & $\begin{array}{c}19.5945 \\
2055\end{array}$ & $46, X Y[20]$ \\
\hline C_67 & 3 & $M$ & MDS/MPN & 0 & $\begin{array}{c}18.4438 \\
3562\end{array}$ & $46, X Y[20]$ \\
\hline C_68 & 75 & $M$ & MDS/MPN & 0 & $\begin{array}{c}9.23835 \\
6164\end{array}$ & $46, X Y[c p 30]$ \\
\hline C_69 & 77 & $M$ & SAML & 0 & $\begin{array}{c}28.7671 \\
2329\end{array}$ & $46, X Y[20]$ \\
\hline C_70 & 59 & $M$ & MDS & 0 & $\begin{array}{c}44.4164 \\
3836\end{array}$ & $46, X Y$ \\
\hline
\end{tabular}




\begin{tabular}{|c|c|c|c|c|c|c|}
\hline C_72 & 54 & $M$ & MDS & 0 & $\begin{array}{c}31.3315 \\
0685\end{array}$ & $46, X Y[20]$ \\
\hline C_73 & 52 & $M$ & MDS & 0 & $\begin{array}{c}48.1972 \\
6027\end{array}$ & $46, X Y[12]$ \\
\hline C_74 & 62 & $\mathrm{~F}$ & MDS & 1 & $\begin{array}{c}30.9041 \\
0959\end{array}$ & $\begin{array}{c}45, X X,- \\
7[8] / 45, \text { idem, } \\
\text { del }(12) \\
\text { (p12)[9]/46,X } \\
\text { X,del }(12) \\
\text { (p12)[1]/46,X } \\
\text { X[2] }\end{array}$ \\
\hline C_75 & 72 & $\mathrm{~F}$ & MDS & 1 & $\begin{array}{c}54.1150 \\
6849\end{array}$ & $46, X X[20]$ \\
\hline C_76 & 63 & $M$ & MDS & 1 & $\begin{array}{c}90.7726 \\
0274\end{array}$ & $\begin{array}{c}4, \mathrm{XY},+8[\mathrm{cp} 1 \\
8] / 46, \mathrm{XY}[2]\end{array}$ \\
\hline C_77 & 75 & $M$ & MDS/MPN & 0 & $\begin{array}{c}15.3534 \\
2466\end{array}$ & $\begin{array}{l}\text { no cyto } \\
\text { available }\end{array}$ \\
\hline C_78 & 73 & $\mathrm{~F}$ & MDS/MPN & 0 & $\begin{array}{c}4.93150 \\
6849\end{array}$ & $46, \mathrm{XX}[20]$ \\
\hline C_79 & 49 & $\mathrm{~F}$ & MDS & 0 & $\begin{array}{c}19.6931 \\
5068\end{array}$ & $\begin{array}{l}47, X X, \\
+21[20]\end{array}$ \\
\hline C_80 & 63 & $M$ & MDS/MPN & 0 & $\begin{array}{c}47.4410 \\
9589\end{array}$ & $46, X Y[20]$ \\
\hline C_81 & 56 & $M$ & $\mathrm{PNH}$ & 0 & $\begin{array}{c}377.654 \\
7945\end{array}$ & $\mathrm{NA}$ \\
\hline C_82 & 46 & $\mathrm{~F}$ & PNH & 0 & $\begin{array}{c}236.778 \\
0822\end{array}$ & $46, \mathrm{XX}[20]$ \\
\hline C_83 & 65 & $M$ & MDS & 0 & $\begin{array}{c}19.5945 \\
2055\end{array}$ & $\begin{array}{l}46, X Y, d e l(5) \\
\text { (q3?1q3?5)[2 } \\
\text { //46,XY[cp18 }\end{array}$ \\
\hline C_84 & 87 & $\mathrm{M}$ & MDS & 0 & $\begin{array}{c}40.7342 \\
4658\end{array}$ & $46, \mathrm{XY}[20]$ \\
\hline C_85 & 85 & $\mathrm{M}$ & sAML & 0 & $\begin{array}{c}22.6191 \\
7808 \\
\end{array}$ & $\begin{array}{c}47, \mathrm{XY},+8[2] / 4 \\
6, \mathrm{XY}[18] \\
\end{array}$ \\
\hline C_89 & 71 & $F$ & MDS & 0 & $\begin{array}{c}19.1671 \\
2329\end{array}$ & $\begin{array}{c}45-46, X X, \text { add } \\
\text { (4) (q21),-- } \\
5[3], p s u \text { dic } \\
\text { (5;?) } \\
\text { (q11.1;?)[16], } \\
\text { add (8) } \\
\text { (p23),-13[17], } \\
\text {-16,add (17) } \\
\text { (p11.2),- } \\
\text { 18,+r[5],+mar } \\
\text { 1,+mar2[cp2 } \\
\text { 0] }\end{array}$ \\
\hline C_90 & 70 & $M$ & MDS & 0 & $\begin{array}{c}66.8712 \\
3288\end{array}$ & $\begin{array}{c}46, X Y, \text { del } \\
(20) \\
(q 11.2 q 13.1)[ \\
3] / 46, X Y[19]\end{array}$ \\
\hline C_91 & 59 & $F$ & MDS & 0 & $\begin{array}{c}17.0301 \\
3699\end{array}$ & $\begin{array}{c}47, \mathrm{XX}, \mathrm{del}(5) \\
\text { (q22q35)[1]/4 } \\
7, \mathrm{sl},+9, \text { add }\end{array}$ \\
\hline
\end{tabular}




\begin{tabular}{|c|c|c|c|c|c|c|}
\hline & & & & & & $\begin{array}{c}(16) \\
(q 24)[3] / 46, X \\
X[17]\end{array}$ \\
\hline C_92 & 82 & $M$ & MDS & 0 & $\begin{array}{c}95.4082 \\
1918\end{array}$ & $46, X Y[20]$ \\
\hline C_93 & 0 & NA & MDS/MPN & NA & NA & NA \\
\hline C_97 & 84 & $F$ & MDS & 0 & $\begin{array}{c}14.8931 \\
5068 \\
\end{array}$ & $46, X Y$ \\
\hline C_98 & 46 & $M$ & MDS & 0 & $\begin{array}{c}29.9506 \\
8493\end{array}$ & $\begin{array}{c}46, X Y,+1, \text { der } \\
(1 ; 7) \\
(q 10 ; \mathrm{p} 10)[9] / \\
46, X Y[3]\end{array}$ \\
\hline C_99 & 61 & $M$ & MDS & 1 & $\begin{array}{c}51.1232 \\
8767\end{array}$ & $46, X Y[20]$ \\
\hline CMML-04 & NA & $\mathrm{F}$ & MDS/MPN & NA & NA & NA \\
\hline $\begin{array}{c}\text { Index13_1 } \\
4\end{array}$ & NA & $\mathrm{M}$ & MDS & NA & NA & $\begin{array}{l}46 \mathrm{XY}, \mathrm{t} \\
(10: 18)\end{array}$ \\
\hline Index6_7 & 62 & $M$ & pAML & NA & NA & $46, X Y$ \\
\hline JMML11 & 13 & $\mathrm{M}$ & MDS/MPN & NA & NA & $46, X Y$ \\
\hline JMML63 & 8 & $\mathrm{~F}$ & MDS/MPN & NA & NA & $48, X X,+8,+19$ \\
\hline JMML72 & 2 & $M$ & MDS/MPN & $\mathrm{NA}$ & NA & $46, X Y$ \\
\hline JMML77 & 1 & $F$ & MDS/MPN & NA & NA & $47, X X,+8$ \\
\hline JMML78 & 1 & $\mathrm{M}$ & MDS/MPN & NA & NA & $46, X Y$ \\
\hline JMML82 & 0 & $F$ & MDS/MPN & NA & NA & $46, X X$ \\
\hline MDS-03 & 45 & $\mathrm{M}$ & MDS & NA & NA & NA \\
\hline MDS-04 & 72 & $M$ & MDS & NA & NA & NA \\
\hline MDS-06 & 73 & $M$ & MDS & $\mathrm{NA}$ & $\mathrm{NA}$ & $\overline{N A}$ \\
\hline MDS-07 & 70 & $M$ & MDS & NA & NA & NA \\
\hline MDS-08 & 75 & $\mathrm{M}$ & MDS & NA & NA & NA \\
\hline MDS-09 & 86 & $M$ & MDS & NA & NA & NA \\
\hline MDS-10 & 25 & $\mathrm{~F}$ & MDS & 1 & 40.4 & NA \\
\hline MDS-11 & 64 & $M$ & MDS & 1 & 38 & NA \\
\hline $\begin{array}{c}\text { MDS1105 } \\
20 \\
\end{array}$ & 73 & $\mathrm{M}$ & MDS & NA & NA & NA \\
\hline MDS-12 & 24 & $F$ & MDS & NA & NA & NA \\
\hline MDS-13 & 40 & $F$ & MDS & $\mathrm{NA}$ & $\mathrm{NA}$ & $\mathrm{NA}$ \\
\hline MDS-14 & 46 & $F$ & MDS & NA & NA & NA \\
\hline MDS-15 & 77 & $M$ & MDS & 1 & 30.8 & NA \\
\hline MDS-16 & 86 & $M$ & MDS & 1 & 37.6 & NA \\
\hline MDS-17 & 73 & $M$ & MDS & 1 & 19.7 & $\mathrm{NA}$ \\
\hline MDS-18 & 75 & $F$ & MDS & NA & NA & $\mathrm{NA}$ \\
\hline MDS-19 & 78 & $F$ & MDS & NA & NA & NA \\
\hline MDS-20 & 56 & $F$ & MDS & NA & NA & NA \\
\hline MDS-21 & 59 & $M$ & MDS & 0 & 15.7 & $\mathrm{NA}$ \\
\hline
\end{tabular}




\begin{tabular}{|c|c|c|c|c|c|c|}
\hline NGS1 & 54 & $\mathrm{~F}$ & sAML & 1 & $\begin{array}{c}17.3917 \\
8082\end{array}$ & $\begin{array}{c}47, X X, \text { del }(5) \\
\text { (q12q33),+?d } \\
\text { el (12) } \\
\text { (q13)[20] }\end{array}$ \\
\hline NGS11 & 59 & $\mathrm{~F}$ & MDS/MPN & 1 & $\begin{array}{c}105.928 \\
7671\end{array}$ & $46, X X[20]$ \\
\hline NGS13 & 78 & $\mathrm{~F}$ & MDS/MPN & 0 & $\begin{array}{c}20.0876 \\
7123\end{array}$ & $47, X X,+8[20]$ \\
\hline NGS15 & 81 & $\mathrm{M}$ & MDS/MPN & 0 & $\begin{array}{c}5.03013 \\
6986\end{array}$ & $\begin{array}{c}45, X Y,- \\
7[10] / 46, X Y, d \\
e l(5) \\
(q 13 q 33)[1] / 4 \\
6, X Y[9] \\
\end{array}$ \\
\hline NGS19 & 77 & $\mathrm{~F}$ & MDS & 0 & $\begin{array}{c}111.780 \\
8219\end{array}$ & $\begin{array}{c}46, \mathrm{XX}, \mathrm{del} \\
(20) \\
(q 11.2)[20]\end{array}$ \\
\hline NGS21 & 72 & $\mathrm{M}$ & MDS & 0 & $\begin{array}{c}7.56164 \\
3836\end{array}$ & $46, X Y$ \\
\hline NGS23 & 64 & $\mathrm{~F}$ & MPN & 1 & $\begin{array}{c}39.0904 \\
1096\end{array}$ & $\begin{array}{c}46, X X \text {, del } \\
(20) \\
(q 11.2)[c p 19] \\
/ 46, X X\end{array}$ \\
\hline NGS25 & 65 & $\mathrm{~F}$ & MDS/MPN & 0 & $\begin{array}{c}164.383 \\
5616\end{array}$ & $46, X X$ \\
\hline NGS27 & NA & $M$ & MDS/MPN & 0 & $\begin{array}{c}18.4438 \\
3562\end{array}$ & $46, X Y[20]$ \\
\hline NGS3 & 77 & $\mathrm{~F}$ & sAML & 0 & $\begin{array}{c}14.7945 \\
2055\end{array}$ & $\begin{array}{c}46, X X, t \\
(8 ; 180 \\
(q 22 ; q 23), t \\
(11 ; 13) \\
(q 21 ; q 12)[c p \\
11] / 46, X X[9] \\
\text { on } 4 / 1 / 09\end{array}$ \\
\hline NGS5 & 63 & $\mathrm{~F}$ & MDS & 1 & $\begin{array}{c}63.5835 \\
6164\end{array}$ & $46, X X$ \\
\hline NGS7 & 68 & $\mathrm{M}$ & MDS/MPN & 0 & $\begin{array}{c}5.26027 \\
3973\end{array}$ & $46, X Y[20]$ \\
\hline NGS9 & 46 & $\mathrm{M}$ & MDS & 1 & $\begin{array}{c}125.589 \\
0411\end{array}$ & $\begin{array}{c}46, X Y, \text { del } \\
(20) \\
(q 11.2 q 13.3)[ \\
18] / 46, X Y[2] .\end{array}$ \\
\hline Nick1 & 74 & $\mathrm{~F}$ & MDS & 0 & $\begin{array}{c}11.0794 \\
5205 \\
\end{array}$ & $46, \mathrm{XX}[11]$ \\
\hline Nick2 & 72 & $\mathrm{M}$ & MDS/MPN & 1 & $\begin{array}{c}52.4054 \\
7945\end{array}$ & $46, X Y[20]$ \\
\hline Nick4 & 68 & $\mathrm{~F}$ & MDS & NA & NA & $46, X X$ \\
\hline Nick5 & 68 & $\mathrm{M}$ & MDS & 0 & $\begin{array}{c}105.534 \\
2466\end{array}$ & $46, X Y[20]$ \\
\hline Nick7 & 68 & $\mathrm{M}$ & MDS & 0 & $\begin{array}{c}11.0136 \\
9863\end{array}$ & $\begin{array}{c}46, X Y, \text { del }(7) \\
(q 22),+8,20, a \\
\text { dd }(21) \\
(p 11.2)[3] / 47,\end{array}$ \\
\hline
\end{tabular}




\begin{tabular}{|c|c|c|c|c|c|c|}
\hline & & & & & & $\begin{array}{c}\text { XY,add (2) } \\
\text { (q31), del (7) } \\
\text { (q22),+8,+8,d } \\
\text { el (11) } \\
\text { (p1?),12,20,a } \\
\text { dd (21) } \\
\text { (p11.2),+mar } \\
\text { 1[12]/47,XY,a } \\
\text { dd (2) } \\
\text { (q31),del (7) } \\
\text { (q22),+8,+8,d } \\
\text { el (11) (p1?)- } \\
12,- \\
21,+ \text { mar1[2]/ } \\
48, X Y, \text { add } \\
\text { (2) (q31)del } \\
\text { (7) } \\
\text { (q22),+8,+8,d } \\
\text { el (11) (p1?),-- } \\
\text { 12,-20,add } \\
\text { (21) } \\
\text { (p11.2),+mar } \\
1,+ \text { mar2[2]. }\end{array}$ \\
\hline Nick8 & 43 & $\mathrm{~F}$ & MDS & 0 & $\begin{array}{c}12.7561 \\
6438\end{array}$ & $\begin{array}{c}46, X X, d e l(9) \\
\text { (q21q22), del } \\
(12) \\
\text { (p12p13)[6]/4 } \\
6, X X, d e l(7) \\
\text { (q22)[4]/46,X } \\
\text { X,del (7) } \\
\text { (q22), del (9) } \\
\text { (q21q22), del } \\
(12) \\
\text { (p12p13)[2]/4 } \\
6, X X[10]\end{array}$ \\
\hline OT052 & NA & $\mathrm{F}$ & MDS & NA & NA & NA \\
\hline OT070 & $\mathrm{NA}$ & $M$ & MDS & NA & NA & $\mathrm{NA}$ \\
\hline P10 & 77 & $\mathrm{~F}$ & MDS & 1 & $\begin{array}{c}58.2575 \\
3425\end{array}$ & $\begin{array}{c}\text { 46,XX,del (5) } \\
\text { (q13q33)[17]/ } \\
46, X X[3]\end{array}$ \\
\hline P11 & 83 & $\mathrm{~F}$ & MDS & 1 & $\begin{array}{c}85.3808 \\
2192\end{array}$ & $\begin{array}{c}46, X X, \mathrm{del}(5) \\
\text { (q13q33)[14]/ } \\
46, X X[6]\end{array}$ \\
\hline P12 & 78 & $\mathrm{~F}$ & MDS & 1 & $\begin{array}{c}71.0465 \\
7534\end{array}$ & $\begin{array}{c}46, X X, \text { del }(5) \\
\text { (q13q33)[6]/4 } \\
6, X X[14]\end{array}$ \\
\hline P15 & 85 & $\mathrm{~F}$ & MDS & 1 & $\begin{array}{c}33.6986 \\
3014\end{array}$ & $\begin{array}{c}46, X X, \text { del }(5) \\
\text { (q13q31)[13]/ } \\
46, X X[7]\end{array}$ \\
\hline P16 & 80 & $\mathrm{~F}$ & MDS & 1 & $\begin{array}{c}67.1671 \\
2329\end{array}$ & $\begin{array}{c}46, X X[20] \\
\text { FISH 5q-: } \\
10.5 \%\end{array}$ \\
\hline
\end{tabular}




\begin{tabular}{|c|c|c|c|c|c|c|}
\hline P17 & 82 & $\mathrm{~F}$ & MDS & 0 & $\begin{array}{c}53.3260 \\
274\end{array}$ & $\begin{array}{c}46, X X, \text { del }(5) \\
\text { (q13q33)[19]/ } \\
46, X X[1]\end{array}$ \\
\hline P18 & 82 & $\mathrm{~F}$ & MDS & 0 & $\begin{array}{c}10.8821 \\
9178\end{array}$ & $\begin{array}{c}46, X X, \text { del }(5) \\
\text { (q13q33)[8]/4 } \\
6, X X[14]\end{array}$ \\
\hline P19 & 62 & $\mathrm{~F}$ & MDS & 1 & $\begin{array}{l}26.0054 \\
7945\end{array}$ & $\begin{array}{c}46, X X, \text { del }(5) \\
\text { (q13q33)[6]/4 } \\
6, X X[11]\end{array}$ \\
\hline P2 & 75 & $\mathrm{~F}$ & MDS & 0 & $\begin{array}{c}60.8219 \\
1781\end{array}$ & $\begin{array}{c}46, X X, \text { del }(5) \\
(q 13 q 33)[7] / 4 \\
6, X X[13]\end{array}$ \\
\hline P20 & NA & & MDS & NA & $\mathrm{NA}$ & $\begin{array}{c}\text { del }(5 q) \text { en } \\
11 / 17 \\
\text { metafases }\end{array}$ \\
\hline P21 & 83 & $\mathrm{M}$ & MDS & 1 & $\begin{array}{c}22.7506 \\
8493\end{array}$ & $\begin{array}{c}46, \mathrm{XY}, \mathrm{del}(5) \\
\text { (q13q33)[17]/ } \\
46, \mathrm{XY}[3]\end{array}$ \\
\hline P22 & 84 & $\mathrm{~F}$ & MDS & 0 & $\begin{array}{c}32.9095 \\
8904\end{array}$ & $\begin{array}{c}46, X Y, \text { del }(5) \\
\text { (q13q33)[1]/4 } \\
6, X X,-3, \text { add } \\
\text { (5) (p15),del } \\
\text { (5) (q13q33),- } \\
\text { 7,add (21) } \\
\text { (q22)[14]/46, } \\
\text { XX[5] }\end{array}$ \\
\hline P23 & 78 & $F$ & MDS & 0 & $\begin{array}{c}10.0931 \\
5068\end{array}$ & $\begin{array}{c}45, \mathrm{XX}, \mathrm{del}(2) \\
\text { (q33q37),- } \\
5[10] / 46, \mathrm{XX}, \mathrm{d} \\
\text { el }(5) \\
(\mathrm{q} 13 \mathrm{q} 33)[3] 4 \\
65, \mathrm{XX},- \\
7[10] / 46, \mathrm{XX},- \\
\text { 14,+der (?)t } \\
(1 ; ?) \\
\text { (p13;?),+mar[ } \\
\text { 10][cp13] }\end{array}$ \\
\hline P24 & 73 & $M$ & MDS & 1 & $\begin{array}{c}25.2493 \\
1507\end{array}$ & $\begin{array}{c}46, X Y, \text { del }(5) \\
\text { (q31q35)[8]/4 } \\
6, X Y[2]\end{array}$ \\
\hline P25 & 65 & $\mathrm{~F}$ & MDS & 1 & $\begin{array}{c}25.1506 \\
8493\end{array}$ & $\begin{array}{l}46, X X, d e l(5) \\
(q 15 q 33)[20]\end{array}$ \\
\hline P26 & 50 & $F$ & MDS & 1 & $\begin{array}{c}15.3534 \\
2466\end{array}$ & $\begin{array}{c}46, \mathrm{XX}, \mathrm{del}(5) \\
\text { (q13q33)[9]/4 } \\
6, \mathrm{XX}[5]\end{array}$ \\
\hline P27 & 81 & $\mathrm{~F}$ & MDS & 1 & $\begin{array}{c}20.2520 \\
5479 \\
\end{array}$ & $\begin{array}{l}46, X Y, \text { del (5) } \\
\text { (q22q35)[20] }\end{array}$ \\
\hline P29 & 70 & $\mathrm{~F}$ & MDS & 1 & $\begin{array}{c}36.3616 \\
4384\end{array}$ & $\begin{array}{c}46, X X, \text { del } \\
(5 q) \\
(q 13 q 33)[16] / \\
46, X X[4]\end{array}$ \\
\hline P3 & 55 & $\mathrm{~F}$ & MDS & 0 & $\begin{array}{c}27.9123 \\
2877 \\
\end{array}$ & $\begin{array}{c}46, X X, t \\
(2 ; 11)\end{array}$ \\
\hline
\end{tabular}




\begin{tabular}{|c|c|c|c|c|c|c|}
\hline & & & & & & $\begin{array}{c}(p 21 ; q 23), \text { del } \\
(5) \\
(q 21 q 33)[15]\end{array}$ \\
\hline P32 & 64 & $M$ & MDS & 1 & $\begin{array}{c}17.7205 \\
4795\end{array}$ & $\begin{array}{c}46, X Y, \text { del }(5) \\
\text { (q13q33)[23]/ } \\
47, X Y, \text { del }(5) \\
(q 13 q 33),+21 \\
{[1] / 46, X Y, \text { del }} \\
(5) \\
\text { (q13q33),del } \\
(X) \\
\text { (q22),+add12 } \\
\text { (p13)[1] }\end{array}$ \\
\hline P33 & 83 & $M$ & MDS & 1 & $\begin{array}{c}12.3945 \\
2055\end{array}$ & $\begin{array}{c}46, X Y, d e l \\
(5 q)[8] / 46, X Y \\
{[12]}\end{array}$ \\
\hline P34 & 63 & $\mathrm{M}$ & MDS & 1 & $\begin{array}{c}20.7452 \\
0548\end{array}$ & $\begin{array}{c}46, X Y, \text { del }(5) \\
\text { (q?q?)[4]/46, } \\
\text { XY[16] }\end{array}$ \\
\hline P35 & 76 & $\mathrm{~F}$ & MDS & 1 & $\begin{array}{c}6.93698 \\
6301\end{array}$ & $\begin{array}{c}\text { SD } 35 \% 5 q- \\
\text { FISH }\end{array}$ \\
\hline P37 & 72 & $\mathrm{~F}$ & MDS & 1 & $\begin{array}{c}32.1863 \\
0137\end{array}$ & $\begin{array}{c}46, \mathrm{XX}, \mathrm{del}(5) \\
(q 13)[19] / 46 \\
\text { XX[1] }\end{array}$ \\
\hline P38 & 82 & $\mathrm{M}$ & MDS & 1 & $\begin{array}{c}11.5068 \\
4932\end{array}$ & $\begin{array}{c}45, X,-Y, \text { del } \\
(5) \\
(q 14)[11] / 46 \\
X Y[3]\end{array}$ \\
\hline P39 & 35 & $\mathrm{~F}$ & MDS & 1 & $\begin{array}{c}81.4684 \\
9315\end{array}$ & $\begin{array}{l}46, X X, d e l(5) \\
\text { (q15q33)[20] }\end{array}$ \\
\hline P4 & 66 & $\mathrm{~F}$ & MDS & 1 & $\begin{array}{c}51.0575 \\
3425\end{array}$ & $\begin{array}{c}\text { 46, XX, del } \\
\text { (5) (q14 q33) } \\
\text { [16]/46, XX } \\
\text { [4] (Jan } \\
\text { 2011) }\end{array}$ \\
\hline P40 & NA & $\mathrm{F}$ & MDS & 1 & $\begin{array}{c}14.7616 \\
4384\end{array}$ & $\begin{array}{c}46, X X, \text { del }(5) \\
(q 12 q 33)[10] / \\
47, X X,+8[3] / 4 \\
6, X X[6]\end{array}$ \\
\hline P41 & 61 & $\mathrm{~F}$ & MDS & 1 & $\begin{array}{c}15.8136 \\
9863\end{array}$ & $\begin{array}{c}46, X Y, \text { del }(5) \\
(q 13 q 33)[1] / 4 \\
6, X X[4]\end{array}$ \\
\hline P42 & 72 & $\mathrm{~F}$ & MDS & 1 & $\begin{array}{c}25.3150 \\
6849\end{array}$ & $\begin{array}{c}46, X X, \mathrm{del}(5) \\
\text { (q13q33)[10]/ } \\
46, \mathrm{XX}[13]\end{array}$ \\
\hline P46 & 65 & $\mathrm{~F}$ & MDS & 1 & $\begin{array}{c}24.7561 \\
6438\end{array}$ & $\begin{array}{c}46, X X, \text { del }(5) \\
\text { (q15q33)[19]/ } \\
46, X X[1]\end{array}$ \\
\hline P47 & 71 & $\mathrm{~F}$ & MDS & 1 & $\begin{array}{c}10.6520 \\
5479\end{array}$ & $\begin{array}{c}46, X X, \text { del }(5) \\
(q 13 q 31)[7] / 4 \\
6, X X[13]\end{array}$ \\
\hline P48 & NA & $\mathrm{F}$ & MDS & 1 & $\begin{array}{c}9.56712 \\
3288\end{array}$ & $\begin{array}{c}46, X X, \text { del } \\
(5 q)[4] / 46, X X\end{array}$ \\
\hline
\end{tabular}




\begin{tabular}{|c|c|c|c|c|c|c|}
\hline P5 & 82 & $F$ & MDS & 0 & $\begin{array}{c}18.4438 \\
3562\end{array}$ & $\begin{array}{c}46, X X, \text { del }(5) \\
(q 13 q 33)[9] / 4 \\
6, X X[11]\end{array}$ \\
\hline P51 & 83 & $M$ & MDS & 1 & $\begin{array}{c}21.8301 \\
3699\end{array}$ & $\begin{array}{c}46, X Y, d e l(5) \\
\text { (q15q33)[9]/4 } \\
6, X Y[11]\end{array}$ \\
\hline P6 & 85 & $M$ & MDS & 0 & $\begin{array}{c}12.7890 \\
411\end{array}$ & $\begin{array}{c}46, \mathrm{XY}, \mathrm{del}(5) \\
\text { (q13q31)[17]/ } \\
46, \mathrm{XY}[3]\end{array}$ \\
\hline P7 & 79 & $M$ & MDS & 1 & $\begin{array}{c}121.446 \\
5753\end{array}$ & $46, X Y$ \\
\hline P8 & 60 & $F$ & MDS & 1 & $\begin{array}{c}50.2684 \\
9315\end{array}$ & $46, X X$ \\
\hline P9 & 79 & $F$ & MDS & 1 & $\begin{array}{c}28.7013 \\
6986\end{array}$ & $\begin{array}{c}46, X X, \text { del }(5) \\
\text { (q13q33)[7]/4 } \\
6, X X[13]\end{array}$ \\
\hline PD4060 & 50 & $F$ & MPN & 1 & 0 & NA \\
\hline PD4063 & NA & $F$ & MPN & NA & NA & NA \\
\hline PD4177 & NA & $F$ & MPN & NA & NA & NA \\
\hline PD4771 & NA & $M$ & MDS & $\mathrm{NA}$ & $\mathrm{NA}$ & $\mathrm{NA}$ \\
\hline PD4772 & NA & $F$ & MPN & NA & NA & NA \\
\hline PD4773 & NA & $F$ & MPN & NA & NA & NA \\
\hline PD4775 & 64 & $F$ & MPN & 0 & NA & $\mathrm{NA}$ \\
\hline PD4776 & NA & $F$ & MPN & $\mathrm{NA}$ & NA & $\mathrm{NA}$ \\
\hline PD4778 & NA & $\mathrm{F}$ & MPN & NA & NA & NA \\
\hline PD4780 & NA & $F$ & MPN & $\mathrm{NA}$ & $\mathrm{NA}$ & $\mathrm{NA}$ \\
\hline PD4943 & NA & $\mathrm{M}$ & MPN & NA & NA & NA \\
\hline PD4944 & NA & $\mathrm{M}$ & MPN & NA & NA & NA \\
\hline PD4990 & NA & $\mathrm{F}$ & MPN & NA & NA & NA \\
\hline PD4996 & NA & $\mathrm{M}$ & MPN & $\mathrm{NA}$ & $\mathrm{NA}$ & $\mathrm{NA}$ \\
\hline PD5003 & NA & $F$ & MPN & NA & NA & NA \\
\hline PD5008 & NA & $F$ & MPN & NA & NA & NA \\
\hline PD5010 & NA & $F$ & MPN & NA & NA & NA \\
\hline PD5029 & NA & $\mathrm{F}$ & MPN & $\mathrm{NA}$ & NA & NA \\
\hline PD5111 & NA & $F$ & MPN & NA & NA & $\mathrm{NA}$ \\
\hline PD5115 & NA & $F$ & MPN & NA & NA & NA \\
\hline PD5116 & NA & $F$ & MPN & NA & NA & NA \\
\hline PD5117 & 64 & $F$ & MPN & 1 & NA & $\mathrm{NA}$ \\
\hline PD5120 & NA & $\mathrm{M}$ & MPN & NA & NA & $\mathrm{NA}$ \\
\hline PD5121 & NA & $F$ & MPN & $\mathrm{NA}$ & NA & $\mathrm{NA}$ \\
\hline PD5122 & NA & $F$ & MPN & NA & NA & NA \\
\hline PD5125 & NA & $F$ & MPN & NA & NA & $\mathrm{NA}$ \\
\hline PD5133 & NA & $\mathrm{NA}$ & MPN & NA & NA & NA \\
\hline PD5134 & NA & $M$ & MPN & NA & NA & NA \\
\hline
\end{tabular}




\begin{tabular}{|c|c|c|c|c|c|c|}
\hline PD5135 & NA & $\mathrm{F}$ & MPN & NA & NA & NA \\
\hline PD5136 & NA & NA & MPN & NA & NA & $\mathrm{NA}$ \\
\hline PD5138 & NA & $F$ & MPN & NA & NA & NA \\
\hline PD5141 & NA & $\mathrm{F}$ & MPN & NA & $\mathrm{NA}$ & $\mathrm{NA}$ \\
\hline PD5151 & NA & $F$ & MPN & NA & NA & NA \\
\hline PD5155 & NA & $\mathrm{F}$ & MPN & NA & NA & $\mathrm{NA}$ \\
\hline PD5158 & NA & $\mathrm{F}$ & MPN & NA & NA & NA \\
\hline PD5161 & NA & $\mathrm{M}$ & MPN & NA & NA & $\mathrm{NA}$ \\
\hline PD5163 & NA & $F$ & MPN & NA & NA & NA \\
\hline PD5164 & NA & $\mathrm{F}$ & MPN & NA & NA & NA \\
\hline PD5165 & NA & $\mathrm{F}$ & MPN & NA & NA & NA \\
\hline PD5175 & NA & $\mathrm{F}$ & MPN & NA & NA & NA \\
\hline PD5182 & NA & $M$ & MPN & NA & NA & NA \\
\hline PD5192 & NA & NA & MPN & NA & NA & NA \\
\hline PD5847 & NA & $\mathrm{F}$ & MPN & NA & NA & NA \\
\hline PD5848 & NA & $\mathrm{F}$ & MPN & $\mathrm{NA}$ & $\mathrm{NA}$ & $\mathrm{NA}$ \\
\hline PD6060 & NA & $M$ & MDS & NA & $\mathrm{NA}$ & $\mathrm{NA}$ \\
\hline PD6068 & NA & NA & MDS & NA & NA & NA \\
\hline PD6077 & 37 & $F$ & MDS & 0 & NA & $46, X X$ \\
\hline PD6100 & NA & $\mathrm{NA}$ & MDS & $\mathrm{NA}$ & $\mathrm{NA}$ & $\mathrm{NA}$ \\
\hline PD6101 & NA & $M$ & MDS & NA & NA & $\mathrm{NA}$ \\
\hline PD6110 & 74 & $\mathrm{~F}$ & MDS & 0 & $\mathrm{NA}$ & $\mathrm{NA}$ \\
\hline PD6116 & NA & NA & MDS & NA & NA & NA \\
\hline PD6122 & NA & $M$ & MDS & NA & NA & $\mathrm{NA}$ \\
\hline PD6127 & NA & $M$ & MDS & 0 & NA & NA \\
\hline PD6193 & NA & $M$ & MDS & $\mathrm{NA}$ & $\mathrm{NA}$ & $\mathrm{NA}$ \\
\hline PD6561 & NA & $M$ & MPN & NA & NA & NA \\
\hline PD6575 & NA & $\mathrm{NA}$ & MPN & NA & NA & NA \\
\hline PD6628 & NA & $F$ & MPN & NA & NA & NA \\
\hline PD6637 & NA & $\mathrm{F}$ & MPN & NA & NA & NA \\
\hline PD6647 & NA & $\mathrm{F}$ & MPN & NA & NA & NA \\
\hline PD6650 & NA & $\mathrm{NA}$ & MPN & NA & NA & NA \\
\hline PD6877 & NA & $M$ & MDS & NA & NA & NA \\
\hline PD6964 & NA & $M$ & MDS & NA & NA & NA \\
\hline PD7084 & NA & $M$ & MDS & NA & NA & $\mathrm{NA}$ \\
\hline PD7089 & NA & NA & MDS & $\mathrm{NA}$ & $\mathrm{NA}$ & $\mathrm{NA}$ \\
\hline PD7092 & NA & $M$ & MDS & NA & NA & NA \\
\hline PD7103 & NA & $M$ & MDS & NA & NA & NA \\
\hline PD7107 & NA & $M$ & MDS & NA & NA & $\mathrm{NA}$ \\
\hline PD7116 & 64 & $M$ & MDS & 1 & NA & $46, X Y$ \\
\hline
\end{tabular}




\begin{tabular}{|c|c|c|c|c|c|c|}
\hline PD7117 & NA & $M$ & MDS & NA & NA & NA \\
\hline PD7118 & NA & NA & MDS & NA & NA & NA \\
\hline PD7120 & NA & $\bar{M}$ & MDS & NA & NA & NA \\
\hline PD7148 & NA & $\mathrm{M}$ & MDS & NA & $\mathrm{NA}$ & $\mathrm{NA}$ \\
\hline PD7151 & NA & $\mathrm{M}$ & MDS & NA & NA & NA \\
\hline PD7152 & NA & $\bar{M}$ & MDS & NA & NA & NA \\
\hline PD7153 & NA & NA & MDS & NA & NA & NA \\
\hline PD7156 & NA & M & MDS & NA & NA & $\mathrm{NA}$ \\
\hline PD7157 & NA & $M$ & MDS & NA & NA & NA \\
\hline PD7158 & NA & M & MDS & NA & NA & NA \\
\hline PD7160 & NA & M & MDS & NA & NA & NA \\
\hline PD7161 & NA & $\mathrm{F}$ & MDS & NA & NA & $\mathrm{NA}$ \\
\hline PD7165 & NA & $M$ & MDS & NA & $\mathrm{NA}$ & $\mathrm{NA}$ \\
\hline PD7166 & NA & $M$ & MDS & NA & NA & NA \\
\hline PD7168 & NA & $\bar{M}$ & MDS & NA & NA & NA \\
\hline PD7222 & NA & $M$ & MDS & $\mathrm{NA}$ & $\mathrm{NA}$ & $\mathrm{NA}$ \\
\hline PD7224 & NA & $M$ & MDS & NA & $\mathrm{NA}$ & NA \\
\hline PD7225 & NA & $M$ & MDS & NA & NA & NA \\
\hline PD7226 & NA & NA & MDS & NA & NA & NA \\
\hline PD7227 & NA & $M$ & MDS & $\mathrm{NA}$ & $\mathrm{NA}$ & $\mathrm{NA}$ \\
\hline PD7280 & NA & $M$ & MPN & NA & NA & NA \\
\hline PD7281 & 59 & $\mathrm{~F}$ & MPN & 1 & $\mathrm{NA}$ & $\mathrm{NA}$ \\
\hline PD7395 & NA & $F$ & MPN & NA & NA & NA \\
\hline PD7396 & NA & $F$ & MPN & NA & NA & $\mathrm{NA}$ \\
\hline PD7398 & NA & NA & MPN & NA & NA & NA \\
\hline PD7399 & NA & $M$ & MPN & $\mathrm{NA}$ & $\mathrm{NA}$ & $\mathrm{NA}$ \\
\hline PD8624 & NA & NA & MPN & NA & NA & NA \\
\hline PD8625 & NA & $F$ & MPN & NA & NA & NA \\
\hline PD8626 & NA & $M$ & MPN & NA & NA & NA \\
\hline PD8627 & NA & $F$ & MPN & NA & NA & $\mathrm{NA}$ \\
\hline PD8628 & NA & $\mathrm{F}$ & MPN & NA & NA & NA \\
\hline PD8629 & NA & $F$ & MPN & NA & NA & NA \\
\hline PD8630 & NA & NA & MPN & NA & NA & NA \\
\hline PD8942 & NA & $F$ & MPN & NA & NA & NA \\
\hline PD8943 & 85 & NA & MPN & 1 & NA & NA \\
\hline PD8944 & NA & $F$ & MPN & $\mathrm{NA}$ & $\mathrm{NA}$ & $\mathrm{NA}$ \\
\hline PD8945 & NA & $M$ & MPN & NA & NA & NA \\
\hline PD8993 & NA & $M$ & MPN & NA & NA & $\mathrm{NA}$ \\
\hline PD8994 & NA & $M$ & MPN & NA & NA & $\mathrm{NA}$ \\
\hline PD9409 & NA & $M$ & MPN & NA & NA & NA \\
\hline
\end{tabular}




\begin{tabular}{|c|c|c|c|c|c|c|}
\hline PD9410 & NA & $M$ & MPN & NA & NA & NA \\
\hline PD9411 & NA & $\mathrm{NA}$ & MPN & $\mathrm{NA}$ & NA & $\mathrm{NA}$ \\
\hline PD9412 & NA & $M$ & MPN & NA & NA & $\mathrm{NA}$ \\
\hline PD9415 & NA & $M$ & MPN & NA & NA & NA \\
\hline PD9418 & NA & $F$ & MPN & NA & NA & NA \\
\hline PD9419 & NA & $M$ & MPN & NA & NA & $\mathrm{NA}$ \\
\hline PD9420 & NA & $F$ & MPN & NA & NA & NA \\
\hline PD9423 & NA & $\mathrm{F}$ & MPN & NA & NA & NA \\
\hline S12_13 & 86 & $F$ & MDS & 0 & 72 & $\begin{array}{c}46, \mathrm{XX}, \mathrm{del}(5) \\
\text { (q22q35)[10]/ } \\
46, \mathrm{XX}[10]\end{array}$ \\
\hline S14_15 & 59 & $\mathrm{~F}$ & pAML & NA & NA & $46, \mathrm{XX}[17]$ \\
\hline S18_19 & NA & $M$ & MDS & NA & NA & $\begin{array}{c}44, X Y,-1, \text { inv } \\
(2) \\
\text { (p23q11.2),- } \\
5, \text { add }(6) \\
\text { (p23),-12,add } \\
\text { (12) (q24.1),- } \\
\text { 14,-16, add } \\
(22) \\
\text { (p11.2),+3ma } \\
r[3] / 45, X,- \\
\text { Y[3]/46,XY[1 } \\
6]\end{array}$ \\
\hline S22_23 & NA & $M$ & MDS & $\mathrm{NA}$ & NA & $46, X Y[20]$ \\
\hline S24_25 & 78 & $M$ & MDS & NA & NA & del5q \\
\hline S26_27 & NA & $M$ & MDS & 0 & $\mathrm{NA}$ & $46 X Y$ \\
\hline S28_29 & NA & $\mathrm{F}$ & MDS & 0 & NA & $\begin{array}{c}47, X X,+6[7] / 4 \\
6, X X[13]\end{array}$ \\
\hline S32_33 & NA & $F$ & MDS/MPN & 0 & NA & $46, \mathrm{XX}[20$ \\
\hline S36_37 & NA & $M$ & MDS & NA & NA & $\begin{array}{c}45-49, X Y, \text { del } \\
\text { (5) (q22q33),- } \\
7, r(7) \\
\text { (p22q36), del } \\
(12) \\
\text { (p12),+16,- } \\
18, \text { del }(20) \\
(q 11.2), \text { del } \\
(22) \\
(q 11.2), 1- \\
2 m a r\end{array}$ \\
\hline S45_46 & NA & $\mathrm{F}$ & MDS/MPN & NA & NA & $\begin{array}{c}46, X X, t \\
(1 ; 11) \\
\text { (p32;q23)[2]/ } \\
47, \text { idem,+8[3] } \\
\text { /46,XX[15 }\end{array}$ \\
\hline $\begin{array}{c}\text { SJAMLM7 } \\
010473 \\
\end{array}$ & 0.81 & $M$ & pAML & NA & NA & $\begin{array}{c}47, \mathrm{XY},+21[9] / \\
46, \mathrm{XY}[21]\end{array}$ \\
\hline
\end{tabular}




\begin{tabular}{|c|c|c|c|c|c|c|}
\hline $\begin{array}{c}\text { SJAMLM7 } \\
010475\end{array}$ & 2 & $M$ & $\mathrm{pAML}$ & NA & NA & $\begin{array}{c}47, X Y, \text { del }(6) \\
\text { (q?16q23),ad } \\
\text { d (7) } \\
(p 22),+21[12] \\
/ 46, X Y[8]\end{array}$ \\
\hline $\begin{array}{c}\text { SJAMLM7 } \\
010480\end{array}$ & 1 & $\mathrm{~F}$ & $\mathrm{pAML}$ & NA & NA & $\begin{array}{c}\text { No } \\
\text { metaphase } \\
\text { available for } \\
\text { analysis }\end{array}$ \\
\hline $\begin{array}{c}\text { SJAMLM7 } \\
010949\end{array}$ & 1 & $M$ & pAML & NA & NA & $\begin{array}{c}46, X Y, t \\
(9 ; 11) \\
(p 22 ; q 23)\end{array}$ \\
\hline $\begin{array}{c}\text { SJAMLM7 } \\
010951\end{array}$ & 1 & $\mathrm{~F}$ & pAML & NA & NA & $\begin{array}{c}46, \mathrm{XX}, \mathrm{t} \\
(8 ; 13) \\
(q 22 ; q 1 ? 4)[8] \\
\text { /48,idem,+6,+ } \\
\text { mar[4]/46,XX[ } \\
\text { 8] }\end{array}$ \\
\hline $\begin{array}{c}\text { SJAMLM7 } \\
010952\end{array}$ & 1 & $M$ & pAML & NA & NA & complex \\
\hline $\begin{array}{c}\text { SJAMLM7 } \\
010960\end{array}$ & 2 & $M$ & pAML & NA & NA & NA \\
\hline $\begin{array}{c}\text { SJAMLM7 } \\
011234\end{array}$ & 0 & $M$ & pAML & NA & NA & $\begin{array}{l}\text { complex } \\
\text { (nog } \\
\text { navragen } \\
\text { Berna) }\end{array}$ \\
\hline $\begin{array}{c}\text { SJAMLM7 } \\
011236\end{array}$ & 1 & $\mathrm{~F}$ & $\mathrm{pAML}$ & NA & NA & $\begin{array}{c}38 \sim 47, \mathrm{XX},- \\
7,- \\
8,+8,+19[\mathrm{cp} 1 \\
4] / 46, \mathrm{XX}[7]\end{array}$ \\
\hline $\begin{array}{c}\text { SJAMLM7 } \\
011238\end{array}$ & 8 & $M$ & pAML & NA & NA & $\begin{array}{c}\text { 46,XY,del } \\
\text { (12) (p11p12) } \\
{[3]}\end{array}$ \\
\hline $\begin{array}{c}\text { SJAMLM7 } \\
011247\end{array}$ & NA & $M$ & pAML & NA & NA & $\begin{array}{c}50, \mathrm{XY}, \mathrm{del}(7) \\
\text { (p13),+8,+19, } \\
+21,+21[10]\end{array}$ \\
\hline $\begin{array}{c}\text { SJAMLM7 } \\
011321\end{array}$ & 3 & $\mathrm{M}$ & pAML & NA & NA & $\begin{array}{c}54, \mathrm{XY}, \mathrm{t} \\
(1 ; 22) \\
(\mathrm{p} 12 ; \mathrm{q} 12),+\mathrm{d} \\
\mathrm{er}(1) \mathrm{t} \\
(1 ; 22),+7,+10 \\
,+14,+19,+21 \\
+21,+21[4] / 4 \\
6, \mathrm{XY}[21] \\
\end{array}$ \\
\hline $\begin{array}{c}\text { SJAMLM7 } \\
011324\end{array}$ & 0 & $M$ & $\mathrm{pAML}$ & NA & NA & $\begin{array}{c}47, \mathrm{XY}, \mathrm{der} \\
\text { (2),der (5),- } \\
7, \mathrm{der} \\
\text { (11),+13or14, } \\
+ \text { mar[5]/46,X } \\
\text { Y[20] }\end{array}$ \\
\hline $\begin{array}{c}\text { SJAMLM7 } \\
011326\end{array}$ & 1 & $M$ & pAML & NA & NA & $46, X Y[15]$ \\
\hline
\end{tabular}




\begin{tabular}{|c|c|c|c|c|c|c|}
\hline $\begin{array}{c}\text { SJAMLM7 } \\
011327\end{array}$ & 1 & $M$ & $\mathrm{pAML}$ & NA & $\mathrm{NA}$ & $\begin{array}{c}46, X Y, \text { der } \\
(13)(q 14), \text { del } \\
(13) \\
(q 13 q ?)[5] / 46 \\
\text {,XY,?t (8;3) } \\
\text { (p1?2;q1?4), } \\
\text { del (13) } \\
(q 14 q ?)[11] / 4 \\
6, X Y[16]\end{array}$ \\
\hline $\begin{array}{c}\text { SJAMLM7 } \\
011328\end{array}$ & 1 & $F$ & $\mathrm{pAML}$ & NA & $\mathrm{NA}$ & $\begin{array}{c}49 \sim 50, \mathrm{XX},+1, \\
+6,+\operatorname{mar} 1,+, \\
\text { mar2 }[\mathrm{cp} 5] / 46, \\
\text { XX[8] }\end{array}$ \\
\hline $\begin{array}{c}\text { SJAMLM7 } \\
015547\end{array}$ & NA & $M$ & pAML & NA & NA & NA \\
\hline $\begin{array}{c}\text { SJAMLM7 } \\
043\end{array}$ & 50 & $M$ & pAML & NA & NA & $\begin{array}{c}46, X Y, t(X ; 1) \\
\text { (q28;q21) }\end{array}$ \\
\hline $\begin{array}{c}\text { SJAMLM7 } \\
044\end{array}$ & 34 & $F$ & $\mathrm{pAML}$ & NA & NA & $46, X X$ \\
\hline $\begin{array}{c}\text { SJAMLM7 } \\
049\end{array}$ & 55 & $M$ & $\mathrm{pAML}$ & NA & NA & $\begin{array}{c}47, \mathrm{XY},+8[14] / \\
46, \mathrm{XY}[7]\end{array}$ \\
\hline $\begin{array}{c}\text { SJAMLM7 } \\
050\end{array}$ & 60 & $\bar{M}$ & $\mathrm{pAML}$ & NA & $\mathrm{NA}$ & $46, X Y$ \\
\hline $\begin{array}{c}\text { SJCBF01 } \\
6\end{array}$ & 10 & $M$ & pAML & $\mathrm{NA}$ & $\mathrm{NA}$ & $\begin{array}{c}48, X Y,+8, i n v \\
(16) \\
(p 13.1 q 22),+ \\
22[12] / 46, X Y[ \\
8]\end{array}$ \\
\hline $\begin{array}{c}\text { SJCBF01 } \\
7\end{array}$ & 7 & $M$ & $\mathrm{pAML}$ & NA & NA & $\begin{array}{c}46, X Y, \text { inv } \\
(16) \\
\text { (p13.1q22)[1 } \\
5] / 46, X Y[5]\end{array}$ \\
\hline $\begin{array}{c}\text { SJCBF02 } \\
7\end{array}$ & 7 & $M$ & $\mathrm{pAML}$ & NA & NA & $\begin{array}{c}46, X Y[13] / 46, \\
X Y, i n v(16) \\
(p 13 q 22)[7] / 4 \\
7, X Y,+22, i n v \\
(16) \\
\text { (p13q22)[2] }\end{array}$ \\
\hline $\begin{array}{c}\text { SJCBF02 } \\
8\end{array}$ & 6 & $F$ & $\mathrm{pAML}$ & NA & NA & $\begin{array}{c}46, X X, t \\
(8 ; 21) \\
(q 22 ; q 22)[12] \\
/ 47, X X,+12, t \\
(8 ; 21) \\
(q 22 ; q 22)[1] / \\
48, X X,+12, t \\
(8 ; 21) \\
(q 22 ; q 22),+d \\
\text { er (21)t }(8 ; 21) \\
(q 22 ; q 22)[1]\end{array}$ \\
\hline $\begin{array}{c}\text { SJCBF02 } \\
9\end{array}$ & 10 & $\mathrm{~F}$ & $\mathrm{pAML}$ & NA & NA & $\begin{array}{c}46, X X[2] / 46, \\
X X, t(8 ; 21) \\
(q 22 ; q 22), \text { del } \\
(9)\end{array}$ \\
\hline
\end{tabular}




\begin{tabular}{|c|c|c|c|c|c|c|}
\hline & & & & & & $\begin{array}{c}\text { (q13q32)[13]/ } \\
47, \text { idem, }+18[ \\
5]\end{array}$ \\
\hline $\begin{array}{c}\text { SJCBF03 } \\
0\end{array}$ & 6 & $F$ & pAML & NA & NA & $\begin{array}{c}46, X X, t \\
(8 ; 21) \\
\text { (q22;q22),der } \\
\text { (9)del (9) } \\
\text { (p13)del (9) } \\
\text { (q13q22)[22]/ } \\
\text { 47,idem,+?18 } \\
\text { [2] }\end{array}$ \\
\hline $\begin{array}{c}\text { SJCBF03 } \\
1\end{array}$ & 6 & $M$ & $\mathrm{pAML}$ & NA & NA & $\begin{array}{c}45, X,-Y, t \\
(8 ; 21) \\
(q 22 ; q 22)[18] \\
/ 46, X Y[2]\end{array}$ \\
\hline $\begin{array}{c}\text { SJCBF03 } \\
3\end{array}$ & 1 & $M$ & $\mathrm{pAML}$ & NA & NA & $\begin{array}{l}\text { Gbanding at } \\
\text { outside } \\
\text { lab/inv (16) } \\
\text { confirmed }\end{array}$ \\
\hline $\begin{array}{c}\text { SJCBF03 } \\
4\end{array}$ & 15 & $\mathrm{~F}$ & $\mathrm{pAML}$ & NA & NA & $\begin{array}{c}\text { Gbanding at } \\
\text { outside lab/t } \\
(8 ; 21) \\
\text { confirmed }\end{array}$ \\
\hline $\begin{array}{c}\text { SJCBF03 } \\
5\end{array}$ & 0 & $M$ & $\mathrm{pAML}$ & NA & NA & $\begin{array}{l}\text { Gbanding at } \\
\text { outside } \\
\text { lab/inv (16) } \\
\text { confirmed }\end{array}$ \\
\hline $\begin{array}{c}\text { SJCBF03 } \\
7\end{array}$ & 10 & $M$ & $\mathrm{pAML}$ & NA & $\mathrm{NA}$ & $\begin{array}{l}\text { Gbanding at } \\
\text { outside } \\
\text { lab/inv (16) } \\
\text { confirmed }\end{array}$ \\
\hline $\begin{array}{c}\text { SJCBF03 } \\
8\end{array}$ & 16 & $F$ & pAML & NA & NA & $\begin{array}{l}\text { Gbanding at } \\
\text { outside } \\
\text { lab/inv (16) } \\
\text { confirmed }\end{array}$ \\
\hline $\begin{array}{c}\text { SJCBF03 } \\
9\end{array}$ & 10 & $M$ & pAML & NA & NA & $\begin{array}{c}\text { Gbanding at } \\
\text { outside lab/t } \\
(8 ; 21) \\
\text { confirmed }\end{array}$ \\
\hline $\begin{array}{c}\text { SJCBF04 } \\
0\end{array}$ & 12 & $F$ & $\mathrm{pAML}$ & NA & $\mathrm{NA}$ & $\begin{array}{c}\text { Gbanding at } \\
\text { outside lab/t } \\
(8 ; 21) \\
\text { confirmed }\end{array}$ \\
\hline $\begin{array}{c}\text { SJCBF04 } \\
1\end{array}$ & 15 & $F$ & pAML & NA & NA & $\begin{array}{l}\text { Gbanding at } \\
\text { outside } \\
\text { lab/inv (16) } \\
\text { confirmed }\end{array}$ \\
\hline $\begin{array}{c}\text { SJCBF04 } \\
2\end{array}$ & 10 & $M$ & $\mathrm{pAML}$ & NA & NA & $\begin{array}{c}\text { Gbanding at } \\
\text { outside lab/t } \\
(8 ; 21) \\
\text { confirmed }\end{array}$ \\
\hline $\begin{array}{c}\text { SJCBF04 } \\
3\end{array}$ & 7 & $F$ & $\mathrm{pAML}$ & NA & NA & $\begin{array}{c}46, \mathrm{XX}[2] / 45 \\
\mathrm{X},-\mathrm{X}, \mathrm{t}\end{array}$ \\
\hline
\end{tabular}




\begin{tabular}{|c|c|c|c|c|c|c|}
\hline & & & & & & $\begin{array}{c}(1 ; 21 ; 8) \\
(p 36 ; q 11- \\
q 21 ; q 22)[19]\end{array}$ \\
\hline $\begin{array}{c}\text { SJCBF04 } \\
4\end{array}$ & 6 & $\mathrm{~F}$ & pAML & NA & NA & $\begin{array}{c}46, X X[1] / 46, \\
X X, t(8 ; 21) \\
(q 22 ; q 22)[10]\end{array}$ \\
\hline $\begin{array}{c}\text { SJCBF09 } \\
0\end{array}$ & 1 & $\mathrm{~F}$ & pAML & NA & NA & $\begin{array}{c}46, X, t(X ; 22) \\
\text { (p22;q11.2),i } \\
\text { nv (16) } \\
\text { (p13.1q22)[2 } \\
3]\end{array}$ \\
\hline $\begin{array}{c}\text { SJCBF09 } \\
1\end{array}$ & 12 & $\mathrm{~F}$ & $\mathrm{pAML}$ & NA & NA & $\begin{array}{c}46, X X, d e r \\
(2) t(2 ; 11) \\
(p 11.2 ; q 21), t \\
(8 ; 21) \\
(q 22 ; q 22), \\
\text { der (11)t } \\
(2 ; 11) \text { del (11) } \\
\text { (p13),i (12) } \\
\text { (q10)[20] }\end{array}$ \\
\hline $\begin{array}{c}\text { SJCBF09 } \\
2\end{array}$ & 9 & $\mathrm{M}$ & $\mathrm{pAML}$ & NA & NA & $\begin{array}{c}46, X Y, \text { inv } \\
(16) \\
\text { (p13.1q22)[1 } \\
\text { 7]/47,idem,+2 } \\
\text { 2[1]/46,XY[2] }\end{array}$ \\
\hline $\begin{array}{c}\text { SJCBF09 } \\
3\end{array}$ & 4 & $\mathrm{M}$ & $\mathrm{pAML}$ & NA & NA & $\begin{array}{c}46, X Y, \text { inv } \\
(16) \\
\text { (p13.1q22)[2 } \\
0]\end{array}$ \\
\hline $\begin{array}{c}\text { SJCBF09 } \\
4\end{array}$ & 4 & $M$ & $\mathrm{pAML}$ & NA & NA & $\begin{array}{c}46, \mathrm{XY}, \mathrm{t} \\
(8 ; 21) \\
(q 22 ; q 22)[19] \\
/ 46, \mathrm{XY}[1]\end{array}$ \\
\hline $\begin{array}{c}\text { SJCBF09 } \\
5\end{array}$ & 7 & $\mathrm{M}$ & pAML & NA & NA & $\begin{array}{c}4 \mathrm{~N}, \mathrm{XXYY}[12] \\
/ 46, X Y[17] / 4 \\
\mathrm{~N}, \mathrm{XXYY} \text {,inv } \\
(16) \\
(\mathrm{p} 13.1 \mathrm{q} 22)[1 \\
2] / 46, \mathrm{XY}[17]\end{array}$ \\
\hline $\begin{array}{c}\text { SJCBF09 } \\
6\end{array}$ & 3 & $M$ & $\mathrm{pAML}$ & NA & NA & $\begin{array}{c}46, X Y, \text { der } \\
\text { (16)ins (16) } \\
\text { (q22p13.1p1 } \\
3.1) \text { del (16) } \\
\text { (q22)[2]/92,id } \\
\text { emx2[8]/46,X } \\
Y[10]\end{array}$ \\
\hline $\begin{array}{c}\text { SJCBF09 } \\
7\end{array}$ & 6 & $\mathrm{M}$ & $\mathrm{pAML}$ & NA & NA & $\begin{array}{c}\text { Gbanding at } \\
\text { outside lab/t } \\
(8 ; 21) \\
\text { confirmed }\end{array}$ \\
\hline $\begin{array}{c}\text { SJCBF09 } \\
8\end{array}$ & 9 & $M$ & $\mathrm{pAML}$ & NA & NA & $\begin{array}{c}46, X,-Y, t \\
(8 ; 21)\end{array}$ \\
\hline
\end{tabular}




\begin{tabular}{|c|c|c|c|c|c|c|}
\hline & & & & & & $\begin{array}{c}(q 22 ; q 22),+8[ \\
20]\end{array}$ \\
\hline $\begin{array}{c}\text { SJCBF09 } \\
9\end{array}$ & 9 & $M$ & $\mathrm{pAML}$ & NA & NA & $\begin{array}{c}\text { Gbanding at } \\
\text { outside lab/t } \\
(8 ; 21) \\
\text { confirmed }\end{array}$ \\
\hline $\begin{array}{c}\text { SJCBF10 } \\
0\end{array}$ & 16 & $F$ & $\mathrm{pAML}$ & NA & $\mathrm{NA}$ & $\begin{array}{c}45, \mathrm{X},-\mathrm{X}, \mathrm{t} \\
(8 ; 21) \\
(\mathrm{q} 22 ; \mathrm{q} 22)[20]\end{array}$ \\
\hline $\begin{array}{c}\text { SJCBF10 } \\
1\end{array}$ & 5 & $\mathrm{~F}$ & $\mathrm{pAML}$ & NA & NA & $\begin{array}{c}46, X X, t \\
(8 ; 21) \\
(q 22 ; q 22)[20]\end{array}$ \\
\hline $\begin{array}{c}\text { SJCBF10 } \\
2\end{array}$ & 15 & $F$ & $\mathrm{pAML}$ & NA & $\mathrm{NA}$ & $\begin{array}{c}\text { Gbanding at } \\
\text { outside lab/t } \\
(8 ; 21) \\
\text { confirmed }\end{array}$ \\
\hline $\begin{array}{c}\text { SJCBF10 } \\
3\end{array}$ & 7 & $M$ & $\mathrm{pAML}$ & NA & NA & $\begin{array}{c}\text { Gbanding at } \\
\text { outside lab/t } \\
(8 ; 21) \\
\text { confirmed }\end{array}$ \\
\hline $\begin{array}{c}\text { SJCBF10 } \\
4\end{array}$ & 17 & $F$ & pAML & NA & $\mathrm{NA}$ & $\begin{array}{c}46, X X, t \\
(16 ; 16) \\
(p 13.1 ; q 22)[2 \\
0]\end{array}$ \\
\hline $\begin{array}{c}\text { SJCBF10 } \\
5\end{array}$ & 18 & $M$ & $\mathrm{pAML}$ & NA & $\mathrm{NA}$ & $\begin{array}{c}46, X Y, \text { inv } \\
(16) \\
\text { (p13.1q22)[2 } \\
\text { 0] }\end{array}$ \\
\hline $\begin{array}{c}\text { SJCBF10 } \\
6\end{array}$ & 1 & $F$ & $\mathrm{pAML}$ & NA & $\mathrm{NA}$ & $\begin{array}{c}46, X X, \text { inv } \\
(16) \\
(p 13.1 q 22)[1 \\
8]\end{array}$ \\
\hline $\begin{array}{c}\text { SJCBF10 } \\
7\end{array}$ & 16 & $M$ & $\mathrm{pAML}$ & NA & $\mathrm{NA}$ & $\begin{array}{c}46, X Y, \text { inv } \\
(16) \\
(p 13.1 q 22)[1 \\
7] / 46, X Y[3]\end{array}$ \\
\hline $\begin{array}{c}\text { SJCBF10 } \\
8\end{array}$ & 4 & $M$ & pAML & NA & $\mathrm{NA}$ & $\begin{array}{c}45, \mathrm{X},-\mathrm{Y}, \mathrm{t} \\
(8 ; 21) \\
(\mathrm{q} 22 ; \mathrm{q} 22)[20]\end{array}$ \\
\hline $\begin{array}{c}\text { SJCBF10 } \\
9\end{array}$ & 15 & $F$ & $\mathrm{pAML}$ & NA & $\mathrm{NA}$ & $\begin{array}{c}46, X X, \text { inv } \\
(16) \\
\text { (p13.1q22)[2 } \\
0]\end{array}$ \\
\hline $\begin{array}{c}\text { SJCBF11 } \\
0\end{array}$ & 7 & $M$ & $\mathrm{pAML}$ & NA & $\mathrm{NA}$ & $\begin{array}{c}45, \mathrm{X},-\mathrm{Y}, \mathrm{t} \\
(8 ; 21) \\
(\mathrm{q} 22 ; \mathrm{q} 22)[17] \\
/ 46, \mathrm{XY}[3]\end{array}$ \\
\hline $\begin{array}{c}\text { SJCBF11 } \\
1\end{array}$ & 15 & $M$ & $\mathrm{pAML}$ & NA & NA & $\begin{array}{c}\text { Gbanding at } \\
\text { outside lab/t } \\
(8 ; 21) \\
\text { confirmed }\end{array}$ \\
\hline $\begin{array}{c}\text { SJCBF11 } \\
2\end{array}$ & 8 & $M$ & pAML & NA & $\mathrm{NA}$ & $\begin{array}{c}46, X Y, t \\
(8 ; 21)\end{array}$ \\
\hline
\end{tabular}




\begin{tabular}{|c|c|c|c|c|c|c|}
\hline & & & & & & $\begin{array}{c}\text { (q22;q22)[19] } \\
/ 46, X Y[1]\end{array}$ \\
\hline $\begin{array}{c}\text { SJCBF11 } \\
3\end{array}$ & 4 & $M$ & $\mathrm{pAML}$ & NA & NA & $\begin{array}{c}\text { 46,XY,inv } \\
(16) \\
(p 13.1 q 22)[1 \\
8] / 46, X Y[2]\end{array}$ \\
\hline $\begin{array}{c}\text { SJCBF11 } \\
4\end{array}$ & 13 & $M$ & $\mathrm{pAML}$ & NA & NA & $\begin{array}{c}\text { Gbanding at } \\
\text { outside } \\
\text { lab/inv (16) } \\
\text { confirmed }\end{array}$ \\
\hline $\begin{array}{c}\text { SJCBF11 } \\
6\end{array}$ & 15 & $M$ & $\mathrm{pAML}$ & NA & NA & $\begin{array}{c}\text { Gbanding at } \\
\text { outside } \\
\text { lab/inv (16) } \\
\text { confirmed }\end{array}$ \\
\hline $\begin{array}{c}\text { SJCBF12 } \\
0\end{array}$ & 15 & $F$ & pAML & NA & NA & $\begin{array}{c}46, \mathrm{XX}, \mathrm{t} \\
(8 ; 21) \\
(\mathrm{q} 22 ; \mathrm{q} 22)[20]\end{array}$ \\
\hline $\begin{array}{c}\text { SJCBF12 } \\
1\end{array}$ & 0 & $F$ & $\mathrm{pAML}$ & NA & NA & $\begin{array}{c}\text { Gbanding at } \\
\text { outside } \\
\text { lab/inv (16) } \\
\text { confirmed }\end{array}$ \\
\hline $\begin{array}{c}\text { SJCBF12 } \\
2\end{array}$ & 18 & $M$ & pAML & $\mathrm{NA}$ & $\mathrm{NA}$ & $\begin{array}{c}46, X Y, \text { inv } \\
(16) \\
(p 13.1 q 22)[1 \\
4] / 46, X Y[6]\end{array}$ \\
\hline $\begin{array}{c}\text { SJCBF12 } \\
3\end{array}$ & 15 & $M$ & $\mathrm{pAML}$ & NA & NA & $\begin{array}{c}\text { Gbanding at } \\
\text { outside } \\
\text { lab/inv (16) } \\
\text { confirmed }\end{array}$ \\
\hline $\begin{array}{c}\text { SJCBF12 } \\
4\end{array}$ & 5 & $M$ & $\mathrm{pAML}$ & NA & NA & $\begin{array}{c}\text { Gbanding at } \\
\text { outside lab/t } \\
(8 ; 21) \\
\text { confirmed }\end{array}$ \\
\hline $\begin{array}{c}\text { SJCBF12 } \\
5\end{array}$ & 10 & $M$ & $\mathrm{pAML}$ & NA & NA & $\begin{array}{c}46, X Y, t \\
(8 ; 21) \\
(q 22 ; q 22)[4] / \\
46, \text { idem,del } \\
(2) \\
(q 37)[2] / 45, i d \\
\text { em,- } \\
\text { Y[4]/45,idem, } \\
- \text { Y,del (2) } \\
\text { (q37)[8] }\end{array}$ \\
\hline $\begin{array}{c}\text { SJCBF13 } \\
7\end{array}$ & 4 & $\mathrm{~F}$ & pAML & NA & NA & $\begin{array}{c}\text { 46,XX,inv } \\
(16) \\
(p 13.1 q 22)[1 \\
0] / 46, X X[11]\end{array}$ \\
\hline $\begin{array}{c}\text { SJCBF13 } \\
8\end{array}$ & 12 & $M$ & $\mathrm{pAML}$ & NA & NA & $\begin{array}{c}45, X,-Y, \text { ins } \\
(8 ; 21) \\
(q 22 ; q 22 q 22) \\
{[30] / 45, X Y,-} \\
7[1]\end{array}$ \\
\hline
\end{tabular}




\begin{tabular}{|c|c|c|c|c|c|c|}
\hline $\begin{array}{c}\text { SJCBF14 } \\
0\end{array}$ & 1 & $M$ & pAML & NA & $\mathrm{NA}$ & $\begin{array}{c}46, X Y, \text { inv } \\
(16) \\
(p 13.1 q 22)[1 \\
1] / 46, X Y[9]\end{array}$ \\
\hline $\begin{array}{c}\text { SJCBF14 } \\
1\end{array}$ & 4 & $F$ & pAML & NA & NA & $\begin{array}{c}\mathrm{t}(8 ; 21) /-7 /- \\
7 \mathrm{Q}\end{array}$ \\
\hline $\begin{array}{c}\text { SJCBF14 } \\
2\end{array}$ & 15 & $M$ & $\mathrm{pAML}$ & NA & NA & $\begin{array}{c}46, X,-Y,+8, t \\
(8 ; 21) \\
(q 22 ; q 22)[19] \\
/ 46, X Y[1]\end{array}$ \\
\hline $\begin{array}{c}\text { SJCBF14 } \\
3\end{array}$ & 14 & $M$ & $\mathrm{pAML}$ & NA & NA & $\begin{array}{c}\text { Gbanding at } \\
\text { outside lab/t } \\
(8 ; 21) \\
\text { confirmed }\end{array}$ \\
\hline $\begin{array}{c}\text { SJCBF14 } \\
4\end{array}$ & 17 & $F$ & $\mathrm{pAML}$ & NA & NA & $\begin{array}{c}\text { Gbanding at } \\
\text { outside } \\
\text { lab/inv (16) } \\
\text { confirmed }\end{array}$ \\
\hline $\begin{array}{c}\text { SJCBF14 } \\
5\end{array}$ & 14 & $F$ & $\mathrm{pAML}$ & NA & NA & $\begin{array}{c}46, \mathrm{XX}, \mathrm{t} \\
(8 ; 21) \\
(\mathrm{q} 22 ; \mathrm{q} 22)[20]\end{array}$ \\
\hline $\begin{array}{c}\text { SJCBF14 } \\
6\end{array}$ & 3 & $M$ & $\mathrm{pAML}$ & NA & $\mathrm{NA}$ & $\begin{array}{c}46, X Y[8] / 45, \\
X,-Y, t(8 ; 21) \\
\text { (q22;q22)[4]/ } \\
46, \text { idem,+der } \\
\text { (21)t }(8 ; 21) \\
\text { (q22;q22)[7] }\end{array}$ \\
\hline $\begin{array}{c}\text { SJCBF14 } \\
7\end{array}$ & 17 & $F$ & $\mathrm{pAML}$ & NA & NA & $\begin{array}{c}46, \mathrm{XX}, \mathrm{t} \\
(8 ; 21) \\
(\mathrm{q} 22 ; \mathrm{q} 22)[12] \\
/ 47, \mathrm{XX}, \mathrm{t} \\
(8 ; 21) \\
(\mathrm{q} 22 ; \mathrm{q} 22),+\mathrm{m} \\
\operatorname{ar}[1]\end{array}$ \\
\hline $\begin{array}{c}\text { SJCBF14 } \\
8\end{array}$ & 18 & $\mathrm{~F}$ & $\mathrm{pAML}$ & NA & NA & $\begin{array}{c}46, X X, \text { inv } \\
(16) \\
\text { (p13q22)[19]/ } \\
47, X X,+22, \text { in } \\
\text { v (16) } \\
\text { (p13q22)[1] }\end{array}$ \\
\hline $\begin{array}{c}\text { SJCBF14 } \\
9\end{array}$ & 4 & $M$ & $\mathrm{pAML}$ & NA & NA & $\begin{array}{c}45, X,-Y, t \\
(3 ; 21 ; 8) \\
(q 27 ; q 22 ; q 22 \\
)[18] / 46, X Y[1]\end{array}$ \\
\hline $\begin{array}{c}\text { SJCBF15 } \\
0\end{array}$ & 2 & $F$ & pAML & NA & NA & $\begin{array}{c}\text { Gbanding at } \\
\text { outside lab/t } \\
(8 ; 21) \\
\text { confirmed }\end{array}$ \\
\hline $\begin{array}{c}\text { SJCBF15 } \\
1\end{array}$ & 17 & $M$ & $\mathrm{pAML}$ & NA & NA & $\begin{array}{c}46, X Y, t \\
(16 ; 16) \\
(p 13.1 ; q 22)[1 \\
9] / 46, X Y[1]\end{array}$ \\
\hline
\end{tabular}




\begin{tabular}{|c|c|c|c|c|c|c|}
\hline $\begin{array}{c}\text { SJCBF15 } \\
2\end{array}$ & 4 & $M$ & pAML & NA & NA & $\begin{array}{l}\text { Gbanding at } \\
\text { outside } \\
\text { lab/inv (16) } \\
\text { confirmed }\end{array}$ \\
\hline tAML-01 & NA & $M$ & sAML & NA & NA & NA \\
\hline tAML-02 & NA & $\mathrm{F}$ & sAML & NA & NA & $\mathrm{NA}$ \\
\hline tAML-03 & NA & $M$ & sAML & NA & NA & NA \\
\hline tAML-04 & NA & $M$ & SAML & NA & NA & NA \\
\hline tAML-05 & NA & $M$ & sAML & NA & NA & $\mathrm{NA}$ \\
\hline tAML-06 & NA & $M$ & sAML & NA & NA & NA \\
\hline tAML-07 & NA & $\mathrm{F}$ & sAML & NA & NA & NA \\
\hline $\begin{array}{l}\text { TARGET- } \\
\text { PAEEYP }\end{array}$ & 1 & $\mathrm{~F}$ & pAML & 0 & 13 & $\begin{array}{c}\text { 46,XX,del } \\
\text { (10) (p13)[30] }\end{array}$ \\
\hline $\begin{array}{l}\text { TARGET- } \\
\text { PAERAH }\end{array}$ & 17 & $M$ & $\mathrm{pAML}$ & 0 & 17 & $\begin{array}{c}46, X Y, \text { inv }(2) \\
(p 11.2 q 13) c, t \\
(8: 21) \\
(q 22: q 22)[20]\end{array}$ \\
\hline $\begin{array}{l}\text { TARGET- } \\
\text { PAKKBK }\end{array}$ & 1 & $\mathrm{~F}$ & $\mathrm{pAML}$ & 0 & 12 & $\begin{array}{c}46, X X,+1, \text { der } \\
(1: 16) \\
(q 10: p 10),-2,- \\
14,+2 \operatorname{mar}[19] \\
\text { /46,XX[1] }\end{array}$ \\
\hline $\begin{array}{l}\text { TARGET- } \\
\text { PAMYAS }\end{array}$ & 10 & $M$ & $\mathrm{pAML}$ & 1 & 108 & $\begin{array}{c}46, X Y[20] . \\
\text { nuc ish } \\
\text { AML1-ETO } \\
\text { positive } \\
\sim 30 \% \text { of cells } \\
\text { consistent } \\
\text { with a t } \\
(8 ; 21) .\end{array}$ \\
\hline $\begin{array}{l}\text { TARGET- } \\
\text { PANGTF }\end{array}$ & 16 & $\mathrm{~F}$ & pAML & $\mathrm{NA}$ & NA & $46, \mathrm{XX}[20]$ \\
\hline $\begin{array}{l}\text { TARGET- } \\
\text { PANLIZ }\end{array}$ & 8 & $M$ & pAML & 0 & 26 & $\begin{array}{c}47, X Y,+8, t \\
(8 ; 21) \\
(q 22 ; q 22)[13] \\
/ 46, X Y[7]\end{array}$ \\
\hline $\begin{array}{l}\text { TARGET- } \\
\text { PANLRE }\end{array}$ & 9 & $M$ & $\mathrm{pAML}$ & 1 & 61 & $\begin{array}{c}46, \mathrm{XY}, \mathrm{t} \\
(8 ; 21) \\
(\mathrm{q} 22 ; \mathrm{q} 22)[20]\end{array}$ \\
\hline $\begin{array}{l}\text { TARGET- } \\
\text { PANSBH }\end{array}$ & 1.3 & $M$ & $\mathrm{pAML}$ & NA & NA & $\begin{array}{c}46, X Y, \text { ider } \\
(16)(q 10) \mathrm{del} \\
(16) \\
(\mathrm{q} 22)[[30]\end{array}$ \\
\hline $\begin{array}{l}\text { TARGET- } \\
\text { PANTWV }\end{array}$ & 14 & $\mathrm{~F}$ & pAML & 0 & 47 & $\begin{array}{c}46, \mathrm{XX}, \mathrm{t} \\
(6 ; 11) \\
\text { (q27;q23)[20] } \\
\text { [cryptic } \\
\text { observed by } \\
\text { FISH only] }\end{array}$ \\
\hline $\begin{array}{l}\text { TARGET- } \\
\text { PANVGP }\end{array}$ & 13 & $M$ & pAML & 1 & 89 & $\begin{array}{c}46, \mathrm{XY}, \mathrm{t} \\
(8 ; 20 ; 21)\end{array}$ \\
\hline
\end{tabular}




\begin{tabular}{|c|c|c|c|c|c|c|}
\hline & & & & & & $\begin{array}{c}\text { (q22;q13.1;q } \\
\text { 22),del (9) } \\
\text { (q22q34)[20] }\end{array}$ \\
\hline $\begin{array}{l}\text { TARGET- } \\
\text { PARGVC }\end{array}$ & 4 & $\mathrm{M}$ & $\mathrm{pAML}$ & 0 & 9 & $\begin{array}{c}45, X,-Y, t \\
(8 ; 21) \\
\text { (q22;q22),ad } \\
\text { d (13) } \\
\text { (p11.2),add } \\
\text { (22) (p11.2) }\end{array}$ \\
\hline $\begin{array}{l}\text { TARGET- } \\
\text { PARIEG }\end{array}$ & 13.4 & $\mathrm{~F}$ & $\mathrm{pAML}$ & NA & NA & $\begin{array}{c}47, \mathrm{XX}, \mathrm{t} \\
(7 ; 11) \\
(\mathrm{p} 15 ; \mathrm{p} 15),+8[ \\
20]\end{array}$ \\
\hline $\begin{array}{l}\text { TARGET- } \\
\text { PARUCB }\end{array}$ & 11 & $\mathrm{M}$ & pAML & 1 & 84 & $\begin{array}{c}47, \mathrm{XY},+21[2] / \\
46, \mathrm{XY}[18]\end{array}$ \\
\hline $\begin{array}{l}\text { TARGET- } \\
\text { PARUNX }\end{array}$ & 6 & $\mathrm{~F}$ & pAML & 1 & 78 & $\begin{array}{c}48, X X,+ \text { der } \\
(6) t(1 ; 6) \\
(q 21 ; q 27), t \\
(7 ; 12) \\
(q 36 ; p 13),+1 \\
9[18][t(7 ; 12) \\
\text { nuc ish ETV6 } \\
\text { sep] }\end{array}$ \\
\hline $\begin{array}{l}\text { TARGET- } \\
\text { PARVUA }\end{array}$ & 15 & $M$ & pAML & 0 & 7 & $47, \mathrm{XY},+8[12]$ \\
\hline $\begin{array}{l}\text { TARGET- } \\
\text { PARWAS }\end{array}$ & 3.6 & $M$ & pAML & NA & NA & $\begin{array}{c}46, X Y, t \\
(9 ; 11) \\
\text { (p22;q23),ad } \\
d(20) \\
\text { (p13)[20] }\end{array}$ \\
\hline $\begin{array}{l}\text { TARGET- } \\
\text { PARWXU }\end{array}$ & 1 & $\mathrm{M}$ & $\mathrm{pAML}$ & 1 & 76 & $\begin{array}{c}46, X Y, \text { del } \\
(11) \\
(p 11.2 p 15)[1 \\
5] / 46, X Y[5]\end{array}$ \\
\hline $\begin{array}{l}\text { TARGET- } \\
\text { PASCGC }\end{array}$ & 14 & $\mathrm{~F}$ & $\mathrm{pAML}$ & 0 & 13 & $\begin{array}{c}46, X X, ? d u p \\
(3) \\
(q 24 q 26.2)[4] \\
146, X X[12]\end{array}$ \\
\hline $\begin{array}{l}\text { TARGET- } \\
\text { PASFEW }\end{array}$ & 1 & $\mathrm{~F}$ & pAML & 0 & 10 & $\begin{array}{c}46, X X, \text { add } \\
(22) \\
(q 13.3)[18] / 4 \\
6, X X[2]\end{array}$ \\
\hline $\begin{array}{l}\text { TARGET- } \\
\text { PASRTP }\end{array}$ & 14 & $\mathrm{~F}$ & $\mathrm{pAML}$ & 1 & 68 & $46, X X[21]$ \\
\hline $\begin{array}{l}\text { TARGET- } \\
\text { PATABB }\end{array}$ & 1 & $\mathrm{M}$ & pAML & 1 & 55 & $\begin{array}{c}46, X Y, d e r \\
(8) t(8 ; 16) \\
\text { (q13;q22),der } \\
\text { (16)inv (16) } \\
\text { (p13.1q22)t } \\
(8 ; 16) \\
(q 13 ; q 22)[4] / \\
47, \text { idem,+22[ } \\
16]\end{array}$ \\
\hline
\end{tabular}




\begin{tabular}{|c|c|c|c|c|c|c|}
\hline $\begin{array}{l}\text { TARGET- } \\
\text { PATELT }\end{array}$ & 12 & $\mathrm{~F}$ & $\mathrm{pAML}$ & 1 & 55 & $\begin{array}{c}46, X X, t \\
(2 ; 11) \\
(q 31 ; p 15)[20]\end{array}$ \\
\hline $\begin{array}{c}\text { TCGA280 } \\
2\end{array}$ & 50 & $\mathrm{M}$ & $\mathrm{pAML}$ & 0 & 12 & $46, X Y[20]$ \\
\hline $\begin{array}{c}\text { TCGA280 } \\
3\end{array}$ & 61 & $\mathrm{~F}$ & pAML & 0 & $\begin{array}{c}26.3835 \\
6164\end{array}$ & Normal \\
\hline $\begin{array}{c}\text { TCGA280 } \\
4\end{array}$ & 30 & $\mathrm{M}$ & $\mathrm{pAML}$ & 1 & $\begin{array}{c}84.3287 \\
6712\end{array}$ & $46, X Y[25]$ \\
\hline $\begin{array}{c}\text { TCGA280 } \\
5\end{array}$ & 77 & $\mathrm{M}$ & pAML & 0 & $\begin{array}{c}18.9369 \\
8631\end{array}$ & $46, X Y[20]$ \\
\hline $\begin{array}{c}\text { TCGA280 } \\
6\end{array}$ & 46 & $M$ & pAML & 0 & $\begin{array}{c}31.3561 \\
6438\end{array}$ & $\mathrm{t}(8 ; 21)$ \\
\hline $\begin{array}{c}\text { TCGA280 } \\
7\end{array}$ & 68 & $\mathrm{~F}$ & pAML & 0 & $\begin{array}{c}5.91788 \\
2192\end{array}$ & $47, X X,+21[24$ \\
\hline $\begin{array}{c}\text { TCGA280 } \\
8\end{array}$ & 23 & $M$ & pAML & 1 & $\begin{array}{c}94.6273 \\
9726\end{array}$ & $47, \mathrm{XXY}[17]$ \\
\hline $\begin{array}{c}\text { TCGA280 } \\
9\end{array}$ & 64 & $F$ & pAML & 0 & $\begin{array}{c}2.38356 \\
1644\end{array}$ & $46, \mathrm{XX}[24]$ \\
\hline $\begin{array}{c}\text { TCGA281 } \\
0\end{array}$ & 76 & $\mathrm{~F}$ & pAML & 0 & $\begin{array}{c}1.19178 \\
8219\end{array}$ & ND \\
\hline $\begin{array}{c}\text { TCGA281 } \\
1\end{array}$ & 81 & $\mathrm{M}$ & $\mathrm{pAML}$ & 0 & $\begin{array}{c}7.98941 \\
9589\end{array}$ & $46, X Y[20]$ \\
\hline $\begin{array}{c}\text { TCGA281 } \\
2\end{array}$ & 25 & $\mathrm{~F}$ & $\mathrm{pAML}$ & 0 & $\begin{array}{c}12.3287 \\
6712\end{array}$ & $46, X X[20]$ \\
\hline $\begin{array}{c}\text { TCGA281 } \\
3\end{array}$ & 78 & $\mathrm{M}$ & pAML & 0 & $\begin{array}{c}1.19178 \\
8219\end{array}$ & $\begin{array}{c}44-45, X,-Y,- \\
5, \text { add }(16) \\
\text { (q22),-17,-- } \\
18, \text { iso } \\
\text { (21), +mars[c } \\
\text { p5]/82- } \\
84, X X,-Y,-3,- \\
4,-11,-12,- \\
19,-- \\
21,+21[\mathrm{cp} 5]\end{array}$ \\
\hline $\begin{array}{c}\text { TCGA281 } \\
4\end{array}$ & 39 & $\mathrm{~F}$ & pAML & 0 & $\begin{array}{c}26.3835 \\
6164\end{array}$ & $\begin{array}{c}46, \mathrm{XX}, \mathrm{del}(5) \\
\text { (q22q33)[4]/4 } \\
6, \mathrm{XX}[16]\end{array}$ \\
\hline $\begin{array}{c}\text { TCGA281 } \\
5\end{array}$ & 49 & $M$ & pAML & 0 & $\begin{array}{c}26.9917 \\
8822\end{array}$ & inv (16) \\
\hline $\begin{array}{c}\text { TCGA281 } \\
6\end{array}$ & 57 & $\mathrm{~F}$ & pAML & 0 & $\begin{array}{c}8.54794 \\
5255\end{array}$ & $46, \mathrm{XX}[20]$ \\
\hline $\begin{array}{c}\text { TCGA281 } \\
7\end{array}$ & 64 & $M$ & $\mathrm{pAML}$ & 0 & $\begin{array}{c}8.97534 \\
2466\end{array}$ & $\begin{array}{c}45, X Y,-7, t \\
(9 ; 22) \\
(q 34 ; q 11.20) \\
{[19] / 46, X Y[1]}\end{array}$ \\
\hline $\begin{array}{c}\text { TCGA281 } \\
8\end{array}$ & 62 & $\mathrm{~F}$ & pAML & 0 & $\begin{array}{c}9.96164 \\
3836\end{array}$ & $\begin{array}{c}46, X X[19] / 46 \\
\text { XX,add (7) } \\
\text { (p?22)[1] }\end{array}$ \\
\hline $\begin{array}{c}\text { TCGA281 } \\
9\end{array}$ & 52 & $\mathrm{~F}$ & pAML & 1 & $\begin{array}{c}82.6273 \\
9726\end{array}$ & $\begin{array}{c}\text { Normal,t } \\
(8 ; 21)\end{array}$ \\
\hline
\end{tabular}




\begin{tabular}{|c|c|c|c|c|c|c|}
\hline $\begin{array}{c}\text { TCGA282 } \\
0\end{array}$ & 76 & $\mathrm{M}$ & pAML & 0 & $\mathrm{NA}$ & $\begin{array}{c}53 \sim 56, X Y,+1, \\
\text { del }(2) \\
(q 33 q 34),+8, \\
+10,+11 \times 2,+ \\
13 \times 1- \\
2,+14, \text { del } \\
(17) \\
(p 11.2),+19, a \\
\text { dd }(21) \\
(q 22),+22[c p \\
20]\end{array}$ \\
\hline $\begin{array}{c}\text { TCGA282 } \\
1\end{array}$ & 64 & $M$ & pAML & 0 & $\begin{array}{c}26.9917 \\
8822\end{array}$ & $\begin{array}{c}47, X Y,+8 \\
{[10] / 46, X Y} \\
{[10]}\end{array}$ \\
\hline $\begin{array}{c}\text { TCGA282 } \\
3 \\
\end{array}$ & 61 & $\mathrm{~F}$ & pAML & 0 & NA & t $(15 ; 17)$ \\
\hline $\begin{array}{c}\text { TCGA282 } \\
4\end{array}$ & 45 & $\mathrm{M}$ & $\mathrm{pAML}$ & 0 & $\begin{array}{c}0.98631 \\
3699\end{array}$ & $46, X Y[20]$ \\
\hline $\begin{array}{c}\text { TCGA282 } \\
5\end{array}$ & 31 & $\mathrm{~F}$ & $\mathrm{pAML}$ & 0 & $\begin{array}{c}6.93698 \\
6314\end{array}$ & 46, XX[20] \\
\hline $\begin{array}{c}\text { TCGA282 } \\
6\end{array}$ & 64 & $\mathrm{~F}$ & $\mathrm{pAML}$ & 0 & $\begin{array}{c}24.3287 \\
6712\end{array}$ & $46, \mathrm{XX}[20]$ \\
\hline $\begin{array}{c}\text { TCGA282 } \\
7\end{array}$ & 33 & $M$ & pAML & 0 & $\begin{array}{c}8.97534 \\
2466\end{array}$ & $\begin{array}{c}\text { 46,XY[18]/46, } \\
\text { XY,del (9) } \\
\text { (q21q33)[1]/4 } \\
6, X Y, d e l(20) \\
\text { (q?12)[1] }\end{array}$ \\
\hline $\begin{array}{c}\text { TCGA282 } \\
8\end{array}$ & 55 & $\mathrm{M}$ & pAML & 1 & $\begin{array}{c}75.9419 \\
5894\end{array}$ & NA \\
\hline $\begin{array}{c}\text { TCGA282 } \\
9\end{array}$ & 50 & $M$ & pAML & 0 & $\begin{array}{c}8.97534 \\
2466\end{array}$ & $\begin{array}{l}\text { N.D.; }-5 q \text { by } \\
\text { FISH }\end{array}$ \\
\hline $\begin{array}{c}\text { TCGA283 } \\
0\end{array}$ & 64 & $\mathrm{~F}$ & $\mathrm{pAML}$ & 0 & $\begin{array}{c}9.41958 \\
942\end{array}$ & $\begin{array}{c}47, X X, i(11) \\
(q 10)[18] / 46 \\
X X[2]\end{array}$ \\
\hline $\begin{array}{c}\text { TCGA283 } \\
1\end{array}$ & 59 & $\bar{M}$ & $\mathrm{pAML}$ & 0 & $\begin{array}{c}21.4195 \\
8941\end{array}$ & $46, X Y[20]$ \\
\hline $\begin{array}{c}\text { TCGA283 } \\
2\end{array}$ & 60 & $\mathrm{~F}$ & $\mathrm{pAML}$ & 0 & 12 & $\begin{array}{c}45, \mathrm{X},- \\
\mathrm{X}[21] / 46, \mathrm{XX}[ \\
9]\end{array}$ \\
\hline $\begin{array}{c}\text { TCGA283 } \\
3\end{array}$ & 77 & $\mathrm{M}$ & $\mathrm{pAML}$ & 0 & $\begin{array}{c}14.9917 \\
8822\end{array}$ & $46, \mathrm{XY}[18]$ \\
\hline $\begin{array}{c}\text { TCGA283 } \\
4\end{array}$ & 33 & M & $\mathrm{pAML}$ & 0 & $\begin{array}{c}8.54794 \\
5255\end{array}$ & $\begin{array}{c}46, X Y, t \\
(11 ; 19) \\
\text { (q23;p13)[17] } \\
/ 46, X Y, t \\
(11 ; 19) \\
\text { (q23;p13),inv } \\
(12) \\
\text { (p12p13)[3] }\end{array}$ \\
\hline $\begin{array}{c}\text { TCGA283 } \\
5\end{array}$ & 48 & $\bar{M}$ & pAML & 1 & $\begin{array}{c}55.2739 \\
7263\end{array}$ & $46, \mathrm{XY}[20]$ \\
\hline $\begin{array}{c}\text { TCGA283 } \\
6\end{array}$ & 35 & $\mathrm{M}$ & pAML & 0 & $\begin{array}{c}16.9972 \\
6274\end{array}$ & $46, \mathrm{XY}[20]$ \\
\hline
\end{tabular}




\begin{tabular}{|c|c|c|c|c|c|c|}
\hline $\begin{array}{c}\text { TCGA283 } \\
7\end{array}$ & 66 & $F$ & pAML & 0 & NA & $46, X X[20]$ \\
\hline $\begin{array}{c}\text { TCGA283 } \\
8\end{array}$ & 67 & $M$ & pAML & 0 & 12 & $\begin{array}{c}46, X Y, \text { del }(5) \\
(q 11.2 q 31),- \\
7,+8,+11, \text { add } \\
(11)(q 23), \text { der } \\
(16 ; 17) \\
(q 10 ; q 10)[18] \\
/ 51, \text { idem, }+1,+ \\
5,+8,+10,+21 \\
{[2]}\end{array}$ \\
\hline $\begin{array}{c}\text { TCGA283 } \\
9\end{array}$ & 51 & $\mathrm{~F}$ & pAML & 0 & $\begin{array}{c}15.9788 \\
2192\end{array}$ & $46, X X[20]$ \\
\hline $\begin{array}{c}\text { TCGA284 } \\
0\end{array}$ & 74 & $\mathrm{M}$ & pAML & 0 & $\begin{array}{c}0.92547 \\
9453\end{array}$ & $\mathrm{t}(15 ; 17)$ \\
\hline $\begin{array}{c}\text { TCGA284 } \\
1\end{array}$ & 51 & $\mathrm{~F}$ & pAML & 0 & $\begin{array}{c}46.6273 \\
9726\end{array}$ & $\mathrm{t}(15 ; 17)$ \\
\hline $\begin{array}{c}\text { TCGA284 } \\
2\end{array}$ & 65 & $M$ & pAML & 0 & NA & $\begin{array}{c}46, X Y, \text { del } \\
(11) \\
\text { (p12)[2]/46,X } \\
Y[18]\end{array}$ \\
\hline $\begin{array}{c}\text { TCGA284 } \\
3\end{array}$ & 77 & $\mathrm{M}$ & pAML & 0 & $\begin{array}{c}7.68493 \\
1568\end{array}$ & $\begin{array}{c}46, X Y, \text { del } \\
(20) \\
(q 11.2)[23] / 9 \\
2, X X Y Y, d e l \\
(20) \\
(q 11.2) \times 2[2] / \\
46, X Y[3]\end{array}$ \\
\hline $\begin{array}{c}\text { TCGA284 } \\
4\end{array}$ & 63 & $\mathrm{M}$ & pAML & 0 & $\begin{array}{c}3.97882 \\
1918\end{array}$ & $\begin{array}{c}46, X Y, t \\
(11 ; 19) \\
(q 23 ; p 13)[20]\end{array}$ \\
\hline $\begin{array}{c}\text { TCGA284 } \\
6\end{array}$ & 57 & $F$ & pAML & 0 & $\begin{array}{c}46.9315 \\
6849\end{array}$ & inv (16) \\
\hline $\begin{array}{c}\text { TCGA284 } \\
7\end{array}$ & 53 & $\mathrm{M}$ & pAML & 0 & $\begin{array}{c}19.9561 \\
6438\end{array}$ & $\begin{array}{c}46, \mathrm{XY}, \mathrm{del}(7) \\
(\mathrm{q} 21 \mathrm{q} 36)[18] / \\
46, \mathrm{XY}[2]\end{array}$ \\
\hline $\begin{array}{c}\text { TCGA284 } \\
8\end{array}$ & 62 & $\mathrm{M}$ & pAML & 0 & $\begin{array}{c}14.9589 \\
4196\end{array}$ & $46, X Y[20]$ \\
\hline $\begin{array}{c}\text { TCGA284 } \\
9\end{array}$ & 39 & $\mathrm{M}$ & pAML & 1 & $\begin{array}{c}72.9863 \\
137\end{array}$ & $\begin{array}{c}\text { 47,XY,del (5) } \\
\text { (q22q33),t } \\
(10 ; 11) \\
(p 13 \sim p 15 ; q 2 \\
2 \sim 23), i(17) \\
(q 10)[3] / 46, X \\
Y[17]\end{array}$ \\
\hline $\begin{array}{c}\text { TCGA285 } \\
0\end{array}$ & 72 & $\mathrm{~F}$ & pAML & 0 & $\begin{array}{c}1.97262 \\
7397\end{array}$ & $\begin{array}{l}\text { Incomplete- } \\
46, X X[2]\end{array}$ \\
\hline $\begin{array}{c}\text { TCGA285 } \\
1\end{array}$ & 66 & $\mathrm{~F}$ & pAML & 0 & $\begin{array}{c}7.95616 \\
4384\end{array}$ & $46, \mathrm{XX}[20]$ \\
\hline $\begin{array}{c}\text { TCGA285 } \\
2\end{array}$ & NA & $\mathrm{M}$ & $\mathrm{pAML}$ & NA & NA & NA \\
\hline $\begin{array}{c}\text { TCGA285 } \\
3 \\
\end{array}$ & 51 & $\mathrm{M}$ & pAML & 0 & $\begin{array}{c}2.89315 \\
6849\end{array}$ & $46, X Y[20]$ \\
\hline
\end{tabular}




\begin{tabular}{|c|c|c|c|c|c|c|}
\hline $\begin{array}{c}\text { TCGA285 } \\
4\end{array}$ & 51 & $\mathrm{~F}$ & pAML & 0 & $\begin{array}{c}12.3287 \\
6712\end{array}$ & $\begin{array}{c}45, \mathrm{Xc}, \mathrm{t}(5 ; 21) \\
\text { (q31;q22)[18] } \\
\text { /45,idem,t } \\
(1 ; 6) \\
\text { (?q21;p23)[1] } \\
\text { /45,Xc[1] }\end{array}$ \\
\hline $\begin{array}{c}\text { TCGA285 } \\
5\end{array}$ & 18 & $\mathrm{M}$ & pAML & 0 & $\begin{array}{c}6.93698 \\
6314\end{array}$ & $\begin{array}{c}48, X Y,+4, t \\
(10 ; 11) \\
(p 13 ; q 21), \text { del } \\
(12) \\
\text { (p11.2),+19[6 } \\
\text { ]/48idem,-- } \\
\text { 17,+i (17) } \\
\text { (q10)[14] }\end{array}$ \\
\hline $\begin{array}{c}\text { TCGA285 } \\
6\end{array}$ & 63 & $M$ & pAML & 0 & $\begin{array}{c}4.89863 \\
137\end{array}$ & $46, X Y[20]$ \\
\hline $\begin{array}{c}\text { TCGA285 } \\
7\end{array}$ & 54 & $\mathrm{M}$ & pAML & 0 & $\begin{array}{c}1.62739 \\
7263\end{array}$ & $\begin{array}{c}42, \mathrm{XY},-5,- \\
\text { 7,add (12) } \\
\text { (p13),t } \\
\text { (14;15) } \\
\text { (q10;q10),der } \\
\text { (17)t (5;17) } \\
\text { (p13;p11.2),- } \\
\text { 18[6]/40,idem } \\
\text {,-11,--add (12) } \\
\text { (p13),der } \\
\text { (12)t (?;12) } \\
\text { (?;p13),- } \\
\text { 19[6]/41,idem } \\
\text {,-der } \\
\text { (17)[3]/41,ide } \\
\text { m,-der } \\
\text { (17),+mar1,+ } \\
\text { mar[3]/41,ide } \\
\text { m,der (1)der } \\
\text { (1) (p12)add } \\
\text { (1) } \\
\text { (p12),+der } \\
\text { (1) (q21)add } \\
\text { (1) (q21),-3,- } \\
\text { 8[2] }\end{array}$ \\
\hline $\begin{array}{c}\text { TCGA285 } \\
8\end{array}$ & 75 & $\mathrm{~F}$ & pAML & 0 & $\begin{array}{c}18.9369 \\
8631\end{array}$ & t $(8 ; 21)$ \\
\hline $\begin{array}{c}\text { TCGA285 } \\
9\end{array}$ & 40 & $M$ & $\mathrm{pAML}$ & 0 & $\begin{array}{c}9.96164 \\
3836\end{array}$ & $46, X Y[20]$ \\
\hline $\begin{array}{c}\text { TCGA286 } \\
0\end{array}$ & 60 & $\mathrm{~F}$ & pAML & 0 & $\begin{array}{c}14.5479 \\
4525\end{array}$ & $\begin{array}{c}44, X X, t \\
(4 ; 11) \\
\text { (q21;q23),-5,-- } \\
7, \text { add (12) } \\
\text { (q24),add } \\
\text { (18) (q23), del } \\
(20) \\
\text { (q12)[3]/43,X }\end{array}$ \\
\hline
\end{tabular}




\begin{tabular}{|c|c|c|c|c|c|c|}
\hline & & & & & & $\begin{array}{c}\text { X,del (3) } \\
\text { (p12),der (3)t } \\
(3 ; 3) \\
\text { (p21;q2?7),p } \\
\text { su dic (5;7) } \\
\text { (q13;p22),- } \\
\text { 10,-15,- } \\
\text { 17,add (18) } \\
\text { (p11.2),- } \\
21,+22,+\operatorname{mar}[ \\
\text { cp14]/46,XX[ } \\
3] \\
\end{array}$ \\
\hline $\begin{array}{c}\text { TCGA286 } \\
1\end{array}$ & 76 & $M$ & pAML & 0 & NA & $\begin{array}{c}47, X Y+8 \\
{[15] / 48, X Y+8} \\
+8[4] / 46, X Y[1 \\
]\end{array}$ \\
\hline $\begin{array}{c}\text { TCGA286 } \\
2\end{array}$ & 33 & $\mathrm{~F}$ & pAML & 1 & $\begin{array}{c}47.1369 \\
8631\end{array}$ & $46, X X[20]$ \\
\hline $\begin{array}{c}\text { TCGA286 } \\
3\end{array}$ & 63 & $\mathrm{M}$ & $\mathrm{pAML}$ & 0 & $\begin{array}{c}1.19178 \\
8219\end{array}$ & $\begin{array}{c}48, X Y,+8,+8[ \\
16] / 46, X Y[4]\end{array}$ \\
\hline $\begin{array}{c}\text { TCGA286 } \\
4\end{array}$ & 54 & $\mathrm{~F}$ & $\mathrm{pAML}$ & 0 & $\begin{array}{c}18.9698 \\
6314\end{array}$ & $\begin{array}{c}47, X X,+8[19] / \\
46, X X[2]\end{array}$ \\
\hline $\begin{array}{c}\text { TCGA286 } \\
5\end{array}$ & 75 & $M$ & pAML & 0 & $\begin{array}{c}2.54794 \\
5255\end{array}$ & $\begin{array}{c}47, X Y,+21[11 \\
] / 48, X Y,+3,+2 \\
1[8]\end{array}$ \\
\hline $\begin{array}{c}\text { TCGA286 } \\
6\end{array}$ & 67 & $M$ & $\mathrm{pAML}$ & 0 & $\begin{array}{c}4.99726 \\
274\end{array}$ & $46, X Y[20]$ \\
\hline $\begin{array}{c}\text { TCGA286 } \\
7\end{array}$ & 66 & $\mathrm{~F}$ & pAML & 0 & $\begin{array}{c}6.49315 \\
6849\end{array}$ & $46, X X[20]$ \\
\hline $\begin{array}{c}\text { TCGA286 } \\
8\end{array}$ & 77 & $\mathrm{M}$ & pAML & 0 & $\begin{array}{c}4.96438 \\
3562\end{array}$ & $\begin{array}{c}46, X Y,- \\
5,+8, \text { del (9) } \\
\text { (q22),add } \\
\text { (10) (q26),der } \\
\text { (15;19) } \\
\text { (q10;q10),ad } \\
\text { d (17) } \\
\text { (p11.2),-20,- } \\
21, \text { add (21) } \\
\text { (p11),add } \\
\text { (22) } \\
\text { (q13),+3mar[ } \\
20]\end{array}$ \\
\hline $\begin{array}{c}\text { TCGA286 } \\
9 \\
\end{array}$ & 64 & $\mathrm{~F}$ & $\mathrm{pAML}$ & 1 & $\begin{array}{c}7.98941 \\
9589\end{array}$ & Normal \\
\hline $\begin{array}{c}\text { TCGA287 } \\
0\end{array}$ & 76 & $\mathrm{M}$ & $\mathrm{pAML}$ & 0 & $\begin{array}{c}4.99726 \\
274\end{array}$ & $\begin{array}{c}\text { Normal,inv } \\
(16)\end{array}$ \\
\hline $\begin{array}{c}\text { TCGA287 } \\
1\end{array}$ & 51 & $\mathrm{M}$ & pAML & 1 & $\begin{array}{c}5.31369 \\
8631\end{array}$ & Normal \\
\hline $\begin{array}{c}\text { TCGA287 } \\
3\end{array}$ & 51 & $\mathrm{~F}$ & pAML & 1 & $\begin{array}{c}8.97534 \\
2466\end{array}$ & Normal \\
\hline $\begin{array}{c}\text { TCGA287 } \\
4\end{array}$ & 59 & $\mathrm{M}$ & pAML & 1 & $\begin{array}{c}13.1917 \\
8822\end{array}$ & $45, X Y,-7[20]$ \\
\hline
\end{tabular}




\begin{tabular}{|c|c|c|c|c|c|c|}
\hline $\begin{array}{c}\text { TCGA287 } \\
5\end{array}$ & 43 & $\bar{M}$ & pAML & 1 & $\begin{array}{c}6.96986 \\
3137\end{array}$ & t $(8 ; 21)$ \\
\hline $\begin{array}{c}\text { TCGA287 } \\
6\end{array}$ & 45 & $\mathrm{~F}$ & pAML & 1 & $\begin{array}{c}4.76712 \\
3288\end{array}$ & $\begin{array}{c}46, X X, 1 \sim 50 \mathrm{~d} \\
\min [12] / 46, \text { id } \\
\text { em,der (6)t } \\
(6 ; ?) \\
(q 22 ; ?)[2] / 46, \\
\text { XX[6] }\end{array}$ \\
\hline $\begin{array}{c}\text { TCGA287 } \\
7\end{array}$ & 60 & $F$ & pAML & 1 & $\begin{array}{c}2.97534 \\
2466\end{array}$ & $\begin{array}{c}46, X X, 9 q h+[2 \\
0]\end{array}$ \\
\hline $\begin{array}{c}\text { TCGA287 } \\
8\end{array}$ & 47 & $\mathrm{~F}$ & pAML & 0 & 12 & $\begin{array}{c}\text { 39 47,XX,del } \\
\text { (5) (q13q33),- } \\
7, \text { der (8)t } \\
\text { (8;?8;8) } \\
\text { (p23;?p11.2p } \\
23 ; q 11.2), d e r \\
\text { (14)t (1;14) } \\
\text { (p12;p11.2)d } \\
\text { er (1)t (7;16) } \\
\text { (p15;q22),+2 } \\
\text { mar[cp19] }\end{array}$ \\
\hline $\begin{array}{c}\text { TCGA287 } \\
9\end{array}$ & 68 & $F$ & pAML & 1 & $\begin{array}{c}8.54794 \\
5255\end{array}$ & $46, X X[20]$ \\
\hline $\begin{array}{c}\text { TCGA288 } \\
0\end{array}$ & 24 & $M$ & pAML & 0 & $\begin{array}{c}13.9397 \\
2627\end{array}$ & 46, XY[20] \\
\hline $\begin{array}{c}\text { TCGA288 } \\
1 \\
\end{array}$ & 48 & $\mathrm{~F}$ & pAML & 1 & $\begin{array}{c}12.9254 \\
7945 \\
\end{array}$ & $\begin{array}{c}\text { Normal,inv } \\
(16)\end{array}$ \\
\hline $\begin{array}{c}\text { TCGA288 } \\
2\end{array}$ & 73 & $F$ & pAML & 0 & 12 & $\begin{array}{c}45, X X,- \\
7[12] / 46, X X[8 \\
]\end{array}$ \\
\hline $\begin{array}{c}\text { TCGA288 } \\
3\end{array}$ & 60 & $M$ & pAML & 1 & 24 & $\begin{array}{c}52 \sim 54, \mathrm{XY},+2, \\
+4,+6,+8, \mathrm{del} \\
(11) \\
(\mathrm{q} 23),+19,+1 \\
9,+21[17] / 46, \\
\mathrm{XY}[3] \\
\end{array}$ \\
\hline $\begin{array}{c}\text { TCGA288 } \\
4\end{array}$ & 44 & $\mathrm{~F}$ & $\mathrm{pAML}$ & 0 & $\begin{array}{c}24.3287 \\
6712 \\
\end{array}$ & $46, X X[19]$ \\
\hline $\begin{array}{l}\text { TCGA288 } \\
5\end{array}$ & 71 & $M$ & pAML & 0 & $\begin{array}{c}7.35616 \\
4384\end{array}$ & $\begin{array}{c}\text { 43,XY-3,del } \\
\text { (5) (q12q33),-- } \\
\text { 7,der (10)t } \\
(10 ; 11) \\
(q 26 ; q 13),- \\
12,- \\
\text { 18,+2mar[20] }\end{array}$ \\
\hline $\begin{array}{c}\text { TCGA288 } \\
6\end{array}$ & 26 & $M$ & pAML & 1 & $\begin{array}{c}5.95684 \\
9316\end{array}$ & t $(8 ; 21)$ \\
\hline $\begin{array}{c}\text { TCGA288 } \\
7 \\
\end{array}$ & 60 & $\mathrm{~F}$ & pAML & 0 & NA & $\begin{array}{c}\text { 46,XX,del (7) } \\
(q 11.2)[20]\end{array}$ \\
\hline $\begin{array}{c}\text { TCGA288 } \\
8 \\
\end{array}$ & 57 & $\mathrm{M}$ & pAML & 1 & $\begin{array}{c}1.98821 \\
9179 \\
\end{array}$ & NA \\
\hline $\begin{array}{c}\text { TCGA289 } \\
0\end{array}$ & 69 & $M$ & pAML & 0 & NA & $\begin{array}{c}47, \mathrm{XY},+9[10] / \\
46, \mathrm{XY}[10]\end{array}$ \\
\hline
\end{tabular}




\begin{tabular}{|c|c|c|c|c|c|c|}
\hline $\begin{array}{c}\text { TCGA289 } \\
1\end{array}$ & 42 & $M$ & $\mathrm{pAML}$ & 0 & $\mathrm{NA}$ & $\begin{array}{c}45, \mathrm{XY}, \operatorname{der}(7) \\
(\mathrm{t}: 7 ; 12) \\
(\mathrm{p} 11.1 ; \mathrm{p} 11.2) \\
,-12,- \\
13,+\operatorname{mar}[19] / \\
46, \mathrm{XY}[1] \\
\end{array}$ \\
\hline $\begin{array}{c}\text { TCGA289 } \\
2\end{array}$ & 42 & $\mathrm{~F}$ & pAML & 1 & $\begin{array}{c}3.96986 \\
3137\end{array}$ & $\begin{array}{c}\text { Normal,inv } \\
(16)\end{array}$ \\
\hline $\begin{array}{c}\text { TCGA289 } \\
3\end{array}$ & 45 & $M$ & $\mathrm{pAML}$ & 0 & $\begin{array}{c}6.96986 \\
3137\end{array}$ & $\begin{array}{c}46, X Y, t \\
(6 ; 11) \\
(q 27 ; q 23)[15]\end{array}$ \\
\hline $\begin{array}{c}\text { TCGA289 } \\
4\end{array}$ & 50 & $\mathrm{~F}$ & $\mathrm{pAML}$ & 0 & $\begin{array}{c}5.91788 \\
2192\end{array}$ & $\begin{array}{c}45, X X,-7, t \\
(9 ; 11) \\
(p 22 ; q 23)[19] \\
/ 46, X X[1]\end{array}$ \\
\hline $\begin{array}{c}\text { TCGA289 } \\
5\end{array}$ & 41 & $\mathrm{~F}$ & pAML & 0 & $\begin{array}{c}5.31369 \\
8631\end{array}$ & N.D. \\
\hline $\begin{array}{c}\text { TCGA289 } \\
6\end{array}$ & 21 & $\mathrm{~F}$ & pAML & 0 & $\begin{array}{c}7.27397 \\
2627 \\
\end{array}$ & $46, \mathrm{XX}[15]$ \\
\hline $\begin{array}{c}\text { TCGA289 } \\
7\end{array}$ & 50 & $\mathrm{~F}$ & pAML & 1 & $\begin{array}{c}7.98941 \\
9589\end{array}$ & $\begin{array}{c}\text { Normal,t } \\
(15 ; 17)\end{array}$ \\
\hline $\begin{array}{c}\text { TCGA289 } \\
8\end{array}$ & 69 & $\mathrm{~F}$ & pAML & 1 & $\begin{array}{c}12.9254 \\
7945\end{array}$ & $47, X X+8[20]$ \\
\hline $\begin{array}{c}\text { TCGA289 } \\
9\end{array}$ & 76 & $\mathrm{~F}$ & $\mathrm{pAML}$ & 0 & $\begin{array}{c}22.6273 \\
9726\end{array}$ & $\begin{array}{c}46, X X+13,21[ \\
\text { cp17]/46,XX[ } \\
3]\end{array}$ \\
\hline $\begin{array}{c}\text { TCGA290 } \\
0\end{array}$ & 70 & $M$ & pAML & 0 & $\begin{array}{c}6.16438 \\
3562\end{array}$ & $46, X Y[20]$ \\
\hline $\begin{array}{c}\text { TCGA290 } \\
1\end{array}$ & 27 & $M$ & pAML & 1 & $\begin{array}{c}1.96849 \\
3157\end{array}$ & $\begin{array}{c}46, X Y, t \\
(9 ; 22) \\
\text { (q34;q11.2[4] } \\
/ 50, \text { idem,+8,+ } \\
\text { 10,+21,+der } \\
\text { (22) (t (9;22) } \\
\text { (q34;q11.2)[1 } \\
6]\end{array}$ \\
\hline $\begin{array}{c}\text { TCGA290 } \\
4\end{array}$ & 65 & $M$ & $\mathrm{pAML}$ & 0 & $\begin{array}{c}16.9643 \\
8356\end{array}$ & $\begin{array}{c}\text { 46 50,XY,- } \\
\text { 1,del (2),del } \\
(5) \\
\text { (q22q33), der } \\
(7) t(1 ; 7) \\
\text { (q21;p13),ad } \\
\text { d (7) } \\
\text { (p11.1),+8,+a } \\
\text { dd (11),del } \\
\text { (12) (p12),del } \\
\text { (13) (q22), } \\
\text { 15,add (17) } \\
\text { (p11.1),- } \\
20,+22 \\
\text { \{cp20\}Compl } \\
\text { ex } \\
\text { Cytogenetics }\end{array}$ \\
\hline
\end{tabular}




\begin{tabular}{|c|c|c|c|c|c|c|}
\hline & & & & & & $\begin{array}{l}\text {, including } \\
5 q^{-},+8\end{array}$ \\
\hline $\begin{array}{c}\text { TCGA290 } \\
8\end{array}$ & 81 & $\mathrm{M}$ & $\mathrm{pAML}$ & 0 & $\begin{array}{c}1.19178 \\
8219\end{array}$ & $\begin{array}{c}6 \sim 49, X Y, \text { del } \\
\text { (3) }(p 14), \text { del } \\
(5) \\
(p 11.2 q 33), d \\
\text { el (17) } \\
(q 21 q 21), \text { add } \\
(21) \\
\text { (p11.2), }+22, \\
\text { mar[cp20] }\end{array}$ \\
\hline $\begin{array}{c}\text { TCGA290 } \\
9\end{array}$ & 22 & $M$ & pAML & 1 & $\begin{array}{c}0.98631 \\
3699\end{array}$ & Normal \\
\hline $\begin{array}{c}\text { TCGA291 } \\
0\end{array}$ & 61 & $\mathrm{~F}$ & $\mathrm{pAML}$ & 0 & NA & $\begin{array}{c}47, X X,+d e r \\
(5) t(2 ; 5) \\
(p 11.2 ; q 11.2) \\
?, t(8 ; 16) \\
(p 11.2 ; p 13.3) \\
{[19]} \\
\end{array}$ \\
\hline $\begin{array}{c}\text { TCGA291 } \\
1\end{array}$ & 51 & $\mathrm{~F}$ & $\mathrm{pAML}$ & 1 & $\begin{array}{c}38.9917 \\
8822\end{array}$ & $\begin{array}{c}\text { 44 46,XX,del } \\
\text { (11) (q23),der } \\
\text { (19)?t (11;;19) } \\
\text { (q23;p13.1)[c } \\
\text { p11]/44 45,X } \\
\text { X,- } \\
\text { 19[cp4]/46,X } \\
\text { X [5] }\end{array}$ \\
\hline $\begin{array}{c}\text { TCGA291 } \\
3\end{array}$ & 61 & $M$ & pAML & 1 & $\begin{array}{c}39.9788 \\
2192\end{array}$ & $\begin{array}{c}\text { Normal,Triso } \\
\text { my } 8\end{array}$ \\
\hline $\begin{array}{c}\text { TCGA291 } \\
4\end{array}$ & 22 & $\mathrm{~F}$ & $\mathrm{pAML}$ & 1 & $\begin{array}{c}26.3835 \\
6164\end{array}$ & $\begin{array}{c}\text { Normal,inv } \\
(16)\end{array}$ \\
\hline $\begin{array}{c}\text { TCGA291 } \\
5\end{array}$ & 73 & $\mathrm{~F}$ & $\mathrm{pAML}$ & 0 & NA & $\begin{array}{c}46, X X, \text { del (3) } \\
\text { (q23q26.2),d } \\
\text { er (7)t (1:7) } \\
\text { (q32;q32), del } \\
(10) \\
\text { (q22q25),t } \\
(13 ; 16) \\
\text { (q34;p11.2)d } \\
\text { up (21) } \\
\text { (q22)[cp20] }\end{array}$ \\
\hline $\begin{array}{c}\text { TCGA291 } \\
6 \\
\end{array}$ & 48 & $\mathrm{~F}$ & $\mathrm{pAML}$ & 1 & $\begin{array}{c}28.9972 \\
6274\end{array}$ & NA \\
\hline $\begin{array}{c}\text { TCGA291 } \\
7\end{array}$ & 41 & $\mathrm{~F}$ & $\mathrm{pAML}$ & 1 & $\begin{array}{c}39.9788 \\
2192\end{array}$ & $\begin{array}{c}46, X X, i(17) \\
(q 10)[1] / 45, s l \\
- \\
7[2] / 48, s l,+13 \\
+19[3] / 46, X X \\
{[15]}\end{array}$ \\
\hline $\begin{array}{c}\text { TCGA291 } \\
9\end{array}$ & 54 & $\mathrm{~F}$ & $\mathrm{pAML}$ & 1 & $\begin{array}{c}2.54794 \\
5255\end{array}$ & Normal \\
\hline $\begin{array}{c}\text { TCGA292 } \\
0\end{array}$ & 44 & $M$ & $\mathrm{pAML}$ & 0 & $\begin{array}{c}12.3287 \\
6712\end{array}$ & $\begin{array}{c}45 \sim 46, X Y, a d \\
d(X)\end{array}$ \\
\hline
\end{tabular}




\begin{tabular}{|c|c|c|c|c|c|c|}
\hline & & & & & & $\begin{array}{c}\text { (q22)[7],Y[4], } \\
\text { der (5)t (5;17) } \\
\text { (q13;21)[18],-- } \\
7[18],+8[17], \\
\text { del (12) } \\
\text { (q23)[16],- } \\
\text { 17[18],add } \\
(18) \\
\text { (p11.2)[14][c } \\
\text { p18] }\end{array}$ \\
\hline $\begin{array}{c}\text { TCGA292 } \\
1\end{array}$ & 56 & $F$ & pAML & 0 & NA & Normal \\
\hline $\begin{array}{c}\text { TCGA292 } \\
2\end{array}$ & 83 & $M$ & $\mathrm{pAML}$ & 0 & NA & Normal \\
\hline $\begin{array}{c}\text { TCGA292 } \\
3\end{array}$ & 78 & $M$ & pAML & 0 & $\begin{array}{c}1.19178 \\
8219\end{array}$ & Normal \\
\hline $\begin{array}{c}\text { TCGA292 } \\
4 \\
\end{array}$ & 59 & $\mathrm{M}$ & pAML & 1 & $\begin{array}{c}2.95894 \\
1959 \\
\end{array}$ & Normal \\
\hline $\begin{array}{c}\text { TCGA292 } \\
5\end{array}$ & 57 & $\mathrm{M}$ & pAML & 0 & $\begin{array}{c}7.95616 \\
4384\end{array}$ & Normal \\
\hline $\begin{array}{c}\text { TCGA292 } \\
8 \\
\end{array}$ & 43 & $\mathrm{~F}$ & pAML & 0 & $\begin{array}{c}1.93972 \\
6274 \\
\end{array}$ & $45, X X,-7[20]$ \\
\hline $\begin{array}{c}\text { TCGA292 } \\
9\end{array}$ & 71 & $\mathrm{~F}$ & pAML & 0 & $\begin{array}{c}4.43835 \\
6164\end{array}$ & $\begin{array}{c}\text { 41 44,X,?i } \\
\text { (X) (p10),- } \\
7, \operatorname{der}(12) t \\
(8 ; 12) \\
(q 11.2 ; p 11.2) \\
,-8 \\
\text { [cp11]/46,XX[ } \\
8[\end{array}$ \\
\hline $\begin{array}{c}\text { TCGA293 } \\
0 \\
\end{array}$ & 63 & $\mathrm{~F}$ & pAML & 0 & NA & Normal \\
\hline $\begin{array}{c}\text { TCGA293 } \\
1\end{array}$ & 75 & $\mathrm{M}$ & pAML & 0 & NA & NA \\
\hline $\begin{array}{c}\text { TCGA293 } \\
2\end{array}$ & 62 & $M$ & pAML & 1 & NA & Normal \\
\hline $\begin{array}{c}\text { TCGA293 } \\
3 \\
\end{array}$ & 58 & $\mathrm{M}$ & pAML & 0 & $\begin{array}{c}4.19589 \\
4196 \\
\end{array}$ & Normal \\
\hline $\begin{array}{c}\text { TCGA293 } \\
4 \\
\end{array}$ & 65 & $M$ & pAML & 1 & $\begin{array}{c}0.92547 \\
9453 \\
\end{array}$ & Normal \\
\hline $\begin{array}{c}\text { TCGA293 } \\
5\end{array}$ & 66 & $\mathrm{M}$ & pAML & 0 & $\begin{array}{c}1.97262 \\
7397\end{array}$ & $\begin{array}{c}44 \sim 47, \mathrm{XY}, \mathrm{del} \\
\text { (5) } \\
\text { (q22q35)[20], } \\
\text {-7[14],-- } \\
\text { 8[6],der (12)t } \\
\text { (10;12) } \\
\text { (p11.2q21)[2] } \\
\text { add (14) } \\
\text { (p12)[11],- } \\
\text { 17[13],der } \\
\text { (17)t (10;17) } \\
\text { (q11.2;p13)[1 } \\
\text { 4],-18[7],add }\end{array}$ \\
\hline
\end{tabular}




\begin{tabular}{|c|c|c|c|c|c|c|}
\hline & & & & & & $\begin{array}{c}(18) \\
(\mathrm{p} 11.2)[7],- \\
21[10], \mathrm{i}(21) \\
(\mathrm{q} 10)[4],- \\
22[4],+\operatorname{mar}[1 \\
0],+\operatorname{mar} 1 \times 2[6 \\
][\mathrm{cp} 20]\end{array}$ \\
\hline $\begin{array}{c}\text { TCGA293 } \\
6\end{array}$ & 61 & $\mathrm{~F}$ & pAML & 1 & $\begin{array}{c}1.93972 \\
6274\end{array}$ & Normal \\
\hline $\begin{array}{c}\text { TCGA293 } \\
7\end{array}$ & 36 & $\mathrm{~F}$ & pAML & 0 & $\begin{array}{c}7.68493 \\
1568\end{array}$ & $\mathrm{t}(8 ; 21)$ \\
\hline $\begin{array}{c}\text { TCGA293 } \\
8\end{array}$ & 76 & $M$ & pAML & 0 & $\begin{array}{c}9.96164 \\
3836\end{array}$ & Normal \\
\hline $\begin{array}{c}\text { TCGA293 } \\
9\end{array}$ & 72 & $\mathrm{M}$ & $\mathrm{pAML}$ & 1 & $\begin{array}{c}14.9589 \\
4196\end{array}$ & $\begin{array}{c}\text { 37 49,XY,+Y } \\
\text {,der (1)add } \\
\text { (1) (p13)del } \\
\text { (1) (q21q25),- } \\
5, \text { der (7)inv } \\
\text { (7) } \\
\text { (p15q11.2)?i } \\
\text { nv (7) } \\
\text { (q22q32),+17 } \\
\text { add (17) } \\
\text { (p13), }+21,+\mathrm{m} \\
\text { ar[cp20] }\end{array}$ \\
\hline $\begin{array}{c}\text { TCGA294 } \\
0\end{array}$ & 35 & $\mathrm{M}$ & pAML & 0 & NA & Normal \\
\hline $\begin{array}{c}\text { TCGA294 } \\
1\end{array}$ & 73 & $M$ & pAML & 0 & NA & $\begin{array}{c}46, X Y, t \\
(9 ; 22) \\
\text { (q34;q11.2)[1 } \\
3] / 34 \sim 37, \text {,ide } \\
\text { m,-3,del (4),- } \\
4,-5,-7,-9,- \\
10, t ? \\
(11 ; 12),-12,- \\
14,-14,-16,- \\
17,- \\
22[\mathrm{cp} 6] / 46, X \\
\text { Y[1] }\end{array}$ \\
\hline $\begin{array}{c}\text { TCGA294 } \\
2\end{array}$ & 67 & $\mathrm{~F}$ & $\mathrm{pAML}$ & 1 & $\begin{array}{c}21.7397 \\
2627\end{array}$ & $\begin{array}{l}\text { Normal,inv } \\
(16)\end{array}$ \\
\hline $\begin{array}{c}\text { TCGA294 } \\
3\end{array}$ & 70 & $\mathrm{~F}$ & pAML & 0 & NA & $\begin{array}{c}46, X X, \text { del (5) } \\
\text { (q11.2q33)[1] } \\
\text { /48 52,idem, } \\
+1,+? \text { del (5) } \\
\text { (q15q33),+11 } \\
\text {,+11,?t } \\
(12 ; 22) \\
(p 13 ; q 12),- \\
13,-17,+i(22) \\
(q 10),+i(22) \\
\text { (q10),+mar[c } \\
\text { p19] }\end{array}$ \\
\hline
\end{tabular}




\begin{tabular}{|c|c|c|c|c|c|c|}
\hline $\begin{array}{c}\text { TCGA294 } \\
4\end{array}$ & 48 & $\mathrm{M}$ & pAML & NA & $\mathrm{NA}$ & $\begin{array}{c}45, X X-7[5]- \\
\text { only } 5 \\
\text { metaphases }\end{array}$ \\
\hline $\begin{array}{c}\text { TCGA294 } \\
5\end{array}$ & 65 & $\mathrm{~F}$ & pAML & 0 & $\begin{array}{c}7.98941 \\
9589\end{array}$ & $\begin{array}{c}46 \sim 47, X X,+i \\
(4)(q 10)[12],- \\
8[5], 10[3][c p 2 \\
0]\end{array}$ \\
\hline $\begin{array}{c}\text { TCGA294 } \\
6\end{array}$ & 41 & $M$ & $\mathrm{pAML}$ & 1 & NA & $\begin{array}{c}\text { incomplete- } \\
46, X Y \text {,del } \\
(12) \\
\text { (p11.20[2]/46 } \\
\text {,XY[3] }\end{array}$ \\
\hline $\begin{array}{c}\text { TCGA294 } \\
7\end{array}$ & 52 & $M$ & pAML & 1 & $\begin{array}{c}1.19178 \\
8219\end{array}$ & $46, X Y[20]$ \\
\hline $\begin{array}{c}\text { TCGA294 } \\
8\end{array}$ & 67 & $M$ & pAML & 0 & $\begin{array}{c}19.6849 \\
3157\end{array}$ & $46, X Y[13]$ \\
\hline $\begin{array}{c}\text { TCGA294 } \\
9\end{array}$ & 58 & $M$ & pAML & 1 & $\begin{array}{c}22.9882 \\
1918\end{array}$ & $\begin{array}{c}47, X Y,+22[10 \\
] / 47, X Y,+8[7] / \\
45, X Y, d e l(3) \\
\text { (p21),del (4) } \\
\text { (p12p15),- } \\
7, ? d u p(7) \\
(q 11.2 q 36)[3]\end{array}$ \\
\hline $\begin{array}{c}\text { TCGA295 } \\
0\end{array}$ & 34 & $\mathrm{~F}$ & pAML & 1 & $\begin{array}{c}1.62739 \\
7263\end{array}$ & t $(8 ; 21)$ \\
\hline $\begin{array}{c}\text { TCGA295 } \\
2\end{array}$ & 60 & $\mathrm{~F}$ & pAML & 0 & $\begin{array}{c}1.19178 \\
8219\end{array}$ & $\begin{array}{c}\text { 44 47,XX,t } \\
(1 ; 15) \\
\text { (q32;q26)[14] } \\
\text {,del (5) } \\
\text { (q13q33)[19], } \\
- \\
\text { 7[20],+8[7],d } \\
\text { el (12) } \\
\text { (p11.2p11.2)[ } \\
\text { 15],del (17) } \\
\text { (q21)[8],der } \\
\text { (22)t (1;22) } \\
\text { (p13;p11.2)[2 } \\
\text { 0],+mar[13][c } \\
\text { p20] }\end{array}$ \\
\hline $\begin{array}{c}\text { TCGA295 } \\
4\end{array}$ & 55 & $F$ & pAML & 1 & $\begin{array}{c}49.5254 \\
7945\end{array}$ & Normal \\
\hline $\begin{array}{c}\text { TCGA295 } \\
5\end{array}$ & 56 & $F$ & pAML & 0 & $\begin{array}{c}16.7671 \\
2329\end{array}$ & Normal \\
\hline $\begin{array}{c}\text { TCGA295 } \\
6\end{array}$ & 61 & $M$ & pAML & 0 & $\begin{array}{c}6.16438 \\
3562\end{array}$ & $\begin{array}{c}\text { Normal,t } \\
(9 ; 11)\end{array}$ \\
\hline $\begin{array}{c}\text { TCGA295 } \\
7\end{array}$ & 31 & $M$ & pAML & 1 & $\begin{array}{c}19.9894 \\
1959\end{array}$ & inv (16) \\
\hline $\begin{array}{c}\text { TCGA295 } \\
9\end{array}$ & 71 & $M$ & pAML & 0 & $\begin{array}{c}16.7671 \\
2329\end{array}$ & $\begin{array}{c}4, X Y,+13[5] \\
46, X Y[15]\end{array}$ \\
\hline TS-MDS-1 & $\mathrm{NA}$ & $M$ & MDS & NA & NA & NA \\
\hline
\end{tabular}




\begin{tabular}{|c|c|c|c|c|c|c|}
\hline $\begin{array}{c}\text { TS-MDS- } \\
10\end{array}$ & NA & $\mathrm{F}$ & MDS & NA & NA & NA \\
\hline TS-MDS-2 & 70 & $M$ & MDS & NA & NA & NA \\
\hline TS-MDS-3 & 78 & $M$ & MDS & NA & NA & NA \\
\hline TS-MDS-4 & NA & $M$ & MDS & NA & NA & $\mathrm{NA}$ \\
\hline TS-MDS-6 & NA & $M$ & MDS & NA & NA & NA \\
\hline TS-MDS-7 & NA & $M$ & MDS & NA & NA & NA \\
\hline TS-MDS-8 & NA & $M$ & MDS & NA & NA & NA \\
\hline TS-MDS-9 & NA & $\mathrm{F}$ & MDS & $\mathrm{NA}$ & $\mathrm{NA}$ & $\mathrm{NA}$ \\
\hline TU007 & NA & $M$ & MDS & NA & NA & $\mathrm{NA}$ \\
\hline TW009 & NA & $\mathrm{F}$ & MDS & NA & NA & NA \\
\hline TW010 & NA & $M$ & MDS & NA & NA & NA \\
\hline TW011 & NA & $M$ & MDS & $\mathrm{NA}$ & NA & $\mathrm{NA}$ \\
\hline TW012 & NA & $\mathrm{F}$ & MDS & NA & $\mathrm{NA}$ & $\mathrm{NA}$ \\
\hline TW013 & NA & $M$ & MDS & NA & NA & NA \\
\hline TW014 & NA & $M$ & MDS & NA & NA & NA \\
\hline TW015 & NA & $M$ & MDS & NA & NA & $\mathrm{NA}$ \\
\hline TW016 & NA & $\mathrm{F}$ & MDS & NA & NA & $\mathrm{NA}$ \\
\hline TW018 & NA & $\mathrm{F}$ & MDS & $\mathrm{NA}$ & NA & $\mathrm{NA}$ \\
\hline TW019 & NA & $\mathrm{F}$ & MDS & NA & NA & NA \\
\hline UPN10 & 84 & $\mathrm{~F}$ & MDS/MPN & $\mathrm{NA}$ & $\mathrm{NA}$ & $46, X X(20)$ \\
\hline UPN11 & 83 & $M$ & MDS/MPN & NA & NA & normal \\
\hline UPN12 & 73 & $\mathrm{~F}$ & MDS/MPN & NA & NA & $46 \mathrm{XX}(20)$ \\
\hline UPN13 & 86 & $\mathrm{~F}$ & MDS/MPN & NA & NA & $46, X X(20)$ \\
\hline UPN14 & 74 & $\mathrm{~F}$ & MDS/MPN & NA & NA & $\begin{array}{l}45, X X, t(4 ; \\
12) \\
(q 13 ; q 12),-7 \\
+\operatorname{mar}(20)\end{array}$ \\
\hline UPN15 & 71 & $\mathrm{~F}$ & MDS/MPN & NA & NA & $\begin{array}{c}46, X X, t(7 ; \\
9)(q 31 ; q 34) \\
{[18] / 46, X X[5]} \\
\text { ish t }(7 ; 9) \\
(\text { wcp7+ } \\
\text { wcp7+) }[5 / 10]\end{array}$ \\
\hline UPN16 & 87 & $M$ & MDS/MPN & NA & NA & $46, X Y(20)$ \\
\hline UPN18 & 74 & $M$ & MDS/MPN & NA & NA & $46, X Y(20)$ \\
\hline UPN19 & 89 & $\mathrm{~F}$ & MDS/MPN & NA & NA & $46, X Y(20)$ \\
\hline UPN2 & 71 & $M$ & MDS/MPN & NA & NA & $46, X Y(20)$ \\
\hline UPN20 & 80 & $\mathrm{~F}$ & MDS/MPN & $\mathrm{NA}$ & NA & $46, X X[20]$ \\
\hline UPN23 & 67 & $M$ & MDS/MPN & $\mathrm{NA}$ & $\mathrm{NA}$ & $46, X Y(20)$ \\
\hline UPN24 & 78 & $\mathrm{M}$ & MDS/MPN & $\mathrm{NA}$ & NA & $\begin{array}{c}46, \\
\text { XY[20],ish } 9 \\
(9 p t e r+, \\
\text { WCP9q+)[30] }\end{array}$ \\
\hline
\end{tabular}




\begin{tabular}{|c|c|c|c|c|c|c|}
\hline UPN25 & 71 & $\mathrm{~F}$ & MDS/MPN & NA & NA & $46, X X(20)$ \\
\hline UPN26 & 63 & $\mathrm{~F}$ & MDS/MPN & NA & NA & $\begin{array}{c}\text { 46, XX[20], } \\
\text { Ish 3q26 } \\
(E V I+)[47] \\
\text { Nuc ish } \\
(E V I 1 \times 2) \\
\left(5^{\prime} E C l 1 \text { sep }\right. \\
\text { 3'EVI1)X1 } \\
\text { [54/200] }\end{array}$ \\
\hline UPN27 & 70 & $M$ & MDS/MPN & NA & NA & $46, X Y(22)$ \\
\hline UPN3 & 85 & $M$ & MDS/MPN & NA & NA & $46, \mathrm{XY}[20]$ \\
\hline UPN31 & 85 & $\mathrm{M}$ & MDS/MPN & NA & NA & $46, X Y(20)$ \\
\hline UPN33 & 80 & $M$ & MDS/MPN & NA & NA & $\begin{array}{c}45, \mathrm{X}- \\
\mathrm{Y}[21] / 46, \mathrm{XY}[ \\
1]\end{array}$ \\
\hline UPN34 & 74 & $\mathrm{~F}$ & MDS/MPN & NA & NA & $\begin{array}{l}\text { 45, XX, -7, } \\
\text { del12p (20) }\end{array}$ \\
\hline UPN35 & 73 & $\mathrm{M}$ & MDS/MPN & NA & NA & normal \\
\hline UPN36 & 48 & $\mathrm{M}$ & MDS/MPN & NA & NA & normal \\
\hline UPN38 & 77 & $\mathrm{M}$ & MDS/MPN & $\mathrm{NA}$ & $\mathrm{NA}$ & $\begin{array}{c}45, \mathrm{X},-\mathrm{Y} \\
{[22 / 26] ;} \\
46, \mathrm{XY}[4 / 26]\end{array}$ \\
\hline UPN39 & 88 & $\mathrm{~F}$ & MDS/MPN & NA & NA & $\begin{array}{c}45, \mathrm{X},- \\
\mathrm{X}[3] / 46, \mathrm{XX}[2 \\
0]\end{array}$ \\
\hline UPN4 & 62 & $\mathrm{M}$ & MDS/MPN & NA & NA & $46 X Y(20)$ \\
\hline UPN40 & 84 & $M$ & MDS/MPN & NA & NA & $46, \mathrm{XY}[28]$ \\
\hline UPN41 & 66 & $\mathrm{M}$ & MDS/MPN & NA & $\mathrm{NA}$ & $\begin{array}{c}46, X Y, \text { inv } \\
\text { (9) (p12q13) } \\
{[20 / 20]}\end{array}$ \\
\hline UPN42 & 83 & $M$ & MDS/MPN & NA & NA & $\begin{array}{c}47, \mathrm{XY},+14<\mathrm{U} \\
+3014>11<\mathrm{U} \\
+3015>/ 46, \mathrm{X} \\
\mathrm{Y}<\mathrm{U}+3014>9 \\
<U+3015>\end{array}$ \\
\hline UPN43 & 59 & $\mathrm{M}$ & MDS/MPN & NA & NA & $\begin{array}{c}44, \mathrm{XY}, \mathrm{del}(5) \\
\text { (q21),add (7) } \\
\text { (q11,2), add } \\
\text { (8) (p21),add } \\
\text { (10) (q22),-- } \\
\text { 13,-18,add } \\
\text { (20) (p13),-- } \\
21 \times 2,- \\
22 \times 2,+4 \mathrm{mar}[ \\
22] / 43, \mathrm{XY},-- \\
4,-5, \text { add (7) } \\
\text { (q11,2), add } \\
\text { (9) (p21),- } \\
10, \text { del (12) } \\
\text { (p12),add }\end{array}$ \\
\hline
\end{tabular}




\begin{tabular}{|c|c|c|c|c|c|c|}
\hline & & & & & & $\begin{array}{c}13)(\mathrm{p} 11,1),- \\
18 \times 2, \text { add } \\
(20)(\mathrm{p} 13),- \\
21,- \\
22 \times 2,+5 \mathrm{mar}[ \\
9] / 46, \mathrm{XY}[1]\end{array}$ \\
\hline UPN44 & 77 & $M$ & MDS/MPN & NA & NA & $46, X Y[27]$ \\
\hline UPN45 & 45 & $\mathrm{~F}$ & MDS/MPN & NA & NA & normal \\
\hline UPN46 & 85 & $M$ & MDS/MPN & NA & NA & $\begin{array}{l}45, X,- \\
Y[4] / 46, \\
X Y[20]\end{array}$ \\
\hline UPN47 & 65 & $\mathrm{~F}$ & MDS/MPN & NA & NA & $\begin{array}{l}46, X X, t(1.3) \\
\text { (p36;q21)[20] }\end{array}$ \\
\hline UPN49 & 71 & $F$ & MDS/MPN & NA & NA & $46, X X[30]$ \\
\hline UPN5 & 66 & $M$ & MDS/MPN & NA & $\mathrm{NA}$ & $\begin{array}{c}47, \mathrm{XY},+ \text { mar } \\
(3) / 46, \mathrm{XY} \\
(17)\end{array}$ \\
\hline UPN6 & 76 & $M$ & MDS/MPN & NA & NA & normal \\
\hline UPN7 & 73 & $M$ & MDS/MPN & NA & NA & $46, X Y(20)$ \\
\hline UPN8 & 70 & $M$ & MDS/MPN & NA & NA & normal \\
\hline UPN9 & 78 & $\mathrm{M}$ & MDS/MPN & NA & NA & $45 X,-Y$ \\
\hline
\end{tabular}

\section{Chapter 2-2: Raw read processing, variant calling, and quality filtering}

All raw read files were first converted to fastq format, then aligned to human genome build hg19 using the Burrows-Wheeler Aligner (BWA) (Li and Durbin 2010). Aligned reads were processed using Samtools ( $\mathrm{Li}$, Handsaker et al. 2009), Picard tools (http://broadinstitute.github.io/picard/), and Genome Analysis Toolkit (GATK) before variant calling by GATK HaplotypeCaller (McKenna, Hanna et al. 2010). GATK best practices were used in processing reads and variant calling. Variants at positions with read depths below eight were omitted from downstream analysis. 
Figure 2: Description of pipeline to call, annotate, and filter variants from fasta files.

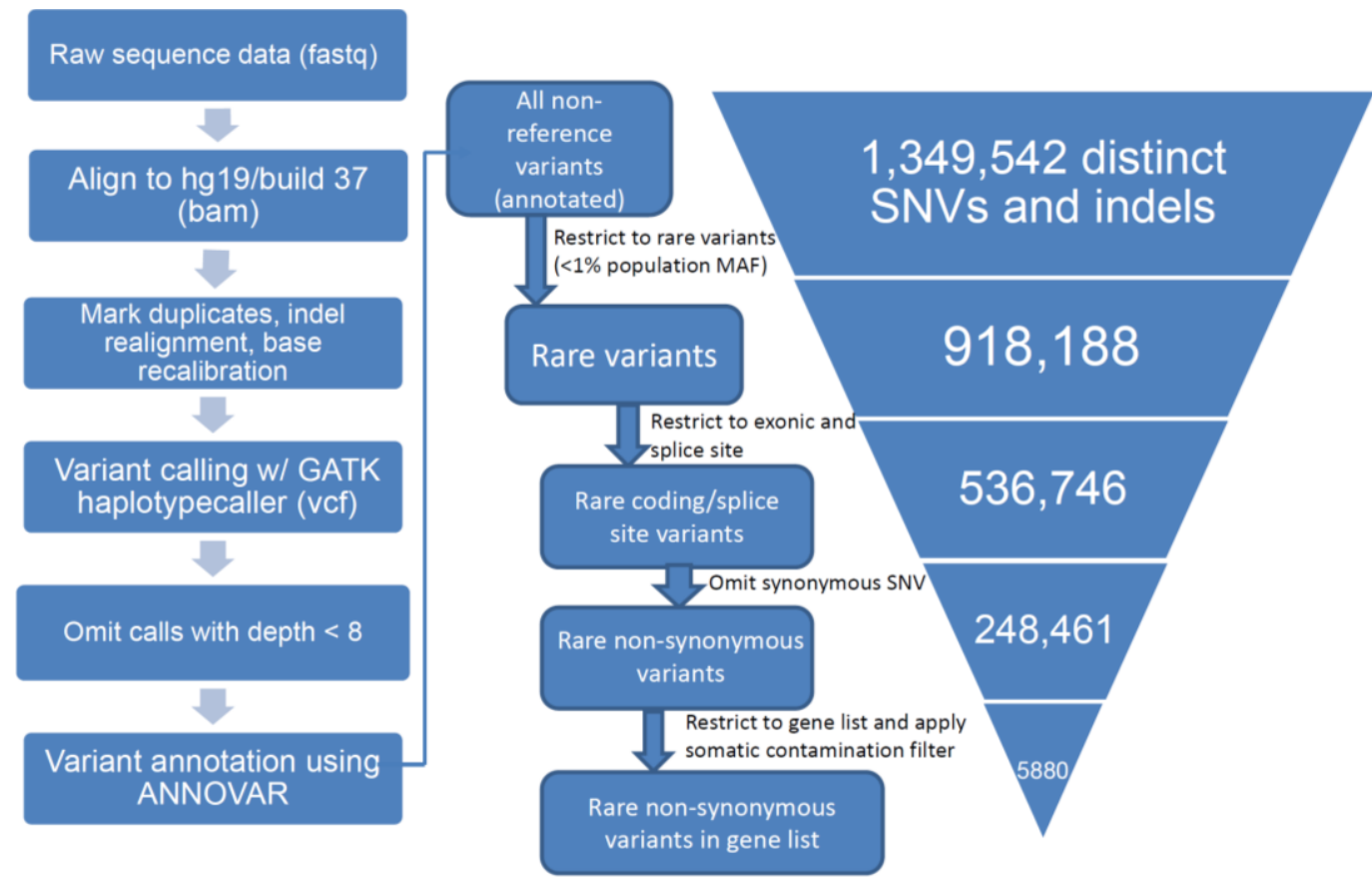

Figure 2: Overview of the variant calling pipeline for this studies. Various filters such as population minor allelic frequency, somatic contamination, and etc were applied to ensure the quality of the called variants.

In order to avoid contamination of malignant cells resulting in false positive germline variants, the variant coordinates were cross-checked with the list of somatic mutations in the same patients, and any commonalities were omitted from the germline list. In addition, we discarded variants with statistically low germline variant allele frequencies (VAFs). We performed a binomial test against the null hypothesis that the variant allele count is drawn from a Binomial $(n, p)$ distribution, where $n$ is the read depth at the locus and $p$ is the expected germline VAF of $50 \%$. Variants corresponding to a Bonferroni-corrected $P$-value below 0.05 , under the alternative hypothesis of $p<0.5$, were discarded. 
Figure 3: Filtering suspected somatic contamination

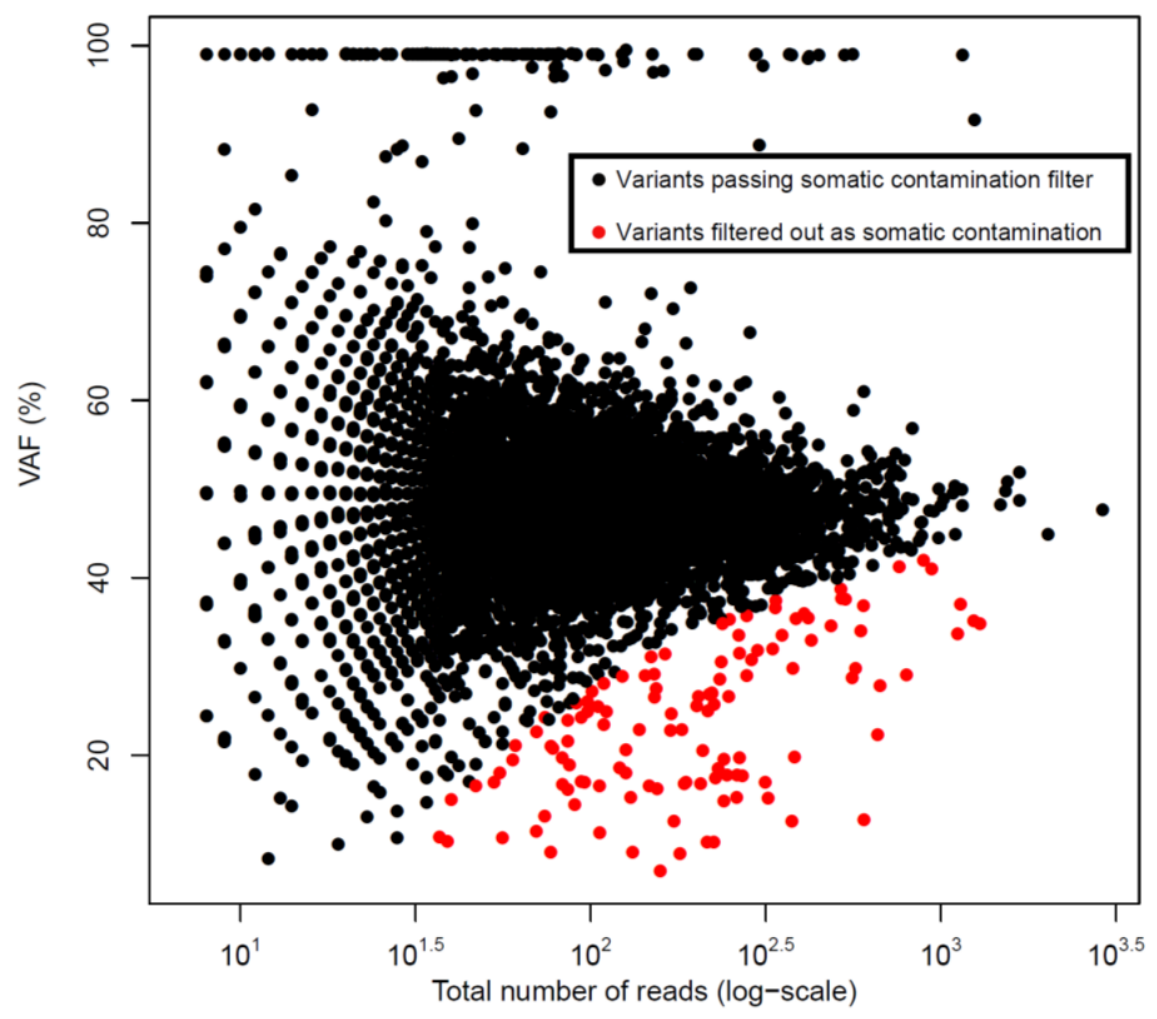

Figure 3: To omit contaminating somatic mutations, we filtered out all variants with allele frequencies (VAFs) that were less than 0.5 at a Bonferroni-corrected $\mathrm{P}<0.05$ significance using a binomial test.

\section{Chapter 2-3: Variant annotation and variant/gene/patient filtering}

ANNOVAR\{Wang, Li et al. 2010\} was used for variant annotation. Rare variants were defined as those with population allele frequencies below $1 \%$ across 1000 genomes (all, European, African, and east Asian), ESP (all, European, and African American), non-1000 genomes Haplotype Reference Consortium (HRC), and ExAC (all, African, east Asian, and non-Finnish European) databases, and frequency below $5 \%$ in our cohort. Only variants annotated as missense, 
nonsense, indel, or splice site were considered in downstream analyses.

Prediction scores from six algorithms - SIFT ( $\mathrm{Ng}$ and Henikoff 2003), Polyphen2 (Adzhubei, Schmidt et al. 2010), MutationTaster\{Schwarz, Cooper et al. 2014\}, MutationAssessor\{Reva, Antipin et al. 2011\}, PROVEAN\{Choi and Chan 2015\}, and CADD\{Rentzsch, Witten et al. 2018) - were each converted into categories "deleterious", "benign", or "uncertain", using thresholds recommended by the developers of each tool. These six categorizations were converted into a hybrid score for each variant, a value between zero and one indicating the proportion of the tools that classify the variant as deleterious, out of those that are not "uncertain". Each variant was also assessed according to the American College of Human Genetics (ACMG) criteria, using ClinVar \{https://www.ncbi.nlm.nih.gov/clinvar/\}. We considered a variant to be pathogenic or likely pathogenic if it was annotated as such (or as a risk factor) and with no annotation as benign, likely benign, or "conflicting interpretations".

Our study focused solely on variants harbored within a curated list of 657 genes (Table 6). This list was assembled from multiple sources, and included genes previously associated with germline susceptibility to cancer\{Rahman 2014\}, [Zhang, Walsh et al. 2015\}, \{Schrader, Cheng et al. 2016\}, \{Grobner, Worst et al. 2018\}, \{Huang, Mashl et al. 2018\} (www.omim.org) and myeloid neoplasms\{Godley and Shimamura 2017\} (www.omim.org), genes known to be recurrently somatically mutated in myeloid malignancies \{Jaiswal, Fontanillas et al. 2014\}, \{Papaemmanuil, Gerstung et al. 2016\}, as well as DNA damage repair 
and hereditary syndrome-associated genes\{Scarpa, Chang et al. 2017\}. Finally, to avoid duplicate or closely related patient samples, we tallied rare variant overlap and mutual exclusivity for all pairs of patients. Any pair with Jaccard index greater than 0.4 were considered duplicate or related, and one of them was discarded.

Table 7: Genes under consideration

\begin{tabular}{|c|c|}
\hline Gene & $\begin{array}{l}\text { Rationale for inclusion } \\
\text { (PubMed ID/ website) }\end{array}$ \\
\hline$A B C B 11$ & $24429628,29489754,29625052$ \\
\hline$A B C D 1$ & OMIM Myeloid Leukemia \\
\hline$A B L 1$ & 27276561, OMIM Myeloid Leukemia \\
\hline$A B L 2$ & OMIM Myeloid Leukemia \\
\hline$A C D$ & 28600339 \\
\hline ACSL6 & OMIM Myeloid Leukemia \\
\hline ACTR5 & 27276561 \\
\hline ACVR1B & OMIM Cancer \\
\hline$A D H 1 B$ & OMIM Cancer \\
\hline AIMP1 & OMIM Myeloid Leukemia \\
\hline AKT1 & OMIM Cancer, 26556299 \\
\hline AKT2 & 26556299 \\
\hline AKT3 & 26556299 \\
\hline$A L D H 2$ & OMIM Cancer \\
\hline$A L K$ & 24429628, 29489754, 29625052, 26556299, 28199314, 26580448 \\
\hline
\end{tabular}




\begin{tabular}{|c|c|}
\hline$A L O X 12 B$ & 26556299 \\
\hline AMER1 & 26556299 \\
\hline ANKRD26 & 28600339 \\
\hline$A P C$ & $\begin{array}{l}\text { OMIM Cancer, 24429628, 29489754, 29625052, 26556299, 28199314, } \\
2658044\end{array}$ \\
\hline$A R$ & OMIM Cancer, 26556299 \\
\hline ARHGAP26 & 27276561, OMIM Myeloid Leukemia \\
\hline$A R I D 1 A$ & 25426837,26556299 \\
\hline$A R I D 1 B$ & 26556299 \\
\hline ASCC1 & OMIM Cancer \\
\hline$A S X L 1$ & 27276561, 25426837, OMIM Myeloid Leukemia, 26556299 \\
\hline ATM & $\begin{array}{l}\text { OMIM Cancer, 24429628, 29489754, 29625052, OMIM Myeloid Leukemia, } \\
26556299,2819931\end{array}$ \\
\hline ATR & OMIM Cancer, 29625052, 26556299, 28199314 \\
\hline ATRX & 27276561, OMIM Myeloid Leukemia, 26556299 \\
\hline ATXN7L1 & 27276561 \\
\hline$A \cup R K A$ & OMIM Cancer \\
\hline AXIN1 & OMIM Cancer, 26556299 \\
\hline AXIN2 & OMIM Cancer, 24429628, 29489754, 29625052, 26556299 \\
\hline$B 2 M$ & 26556299 \\
\hline $\mathrm{BACH1}$ & 28600339 \\
\hline$B A P 1$ & $\begin{array}{l}\text { OMIM Cancer, 24429628, 29489754, 29625052, 26556299, 28199314, } \\
2658044\end{array}$ \\
\hline$B A R D 1$ & OMIM Cancer, 26556299, 28199314 \\
\hline$B A X$ & OMIM Cancer, OMIM Myeloid Leukemia \\
\hline BCAP31 & OMIM Myeloid Leukemia \\
\hline
\end{tabular}




\begin{tabular}{|c|c|}
\hline$B C L 10$ & OMIM Cancer, 25426837 \\
\hline$B C L 11 B$ & 25426837 \\
\hline BCL2 & OMIM Myeloid Leukemia \\
\hline$B C L 3$ & OMIM Myeloid Leukemia \\
\hline$B C L 6$ & 25426837 \\
\hline$B C O R$ & $27276561,25426837,26556299$ \\
\hline BCORL1 & 25426837 \\
\hline$B C R$ & OMIM Myeloid Leukemia \\
\hline BIRC3 & 25426837 \\
\hline$B L M$ & $24429628,29489754,29625052,26556299,28199314$ \\
\hline BMPR1A & $24429628,29489754,29625052,26556299,28199314,26580448$ \\
\hline$B R A F$ & $\begin{array}{l}\text { OMIM Cancer, 27276561, 29489754, 29625052, 25426837, 26556299, } \\
26580448\end{array}$ \\
\hline$B R C A 1$ & $\begin{array}{l}\text { OMIM Cancer, 24429628, 28600339, 29489754, 29625052, 26556299, } \\
28199314,2658044\end{array}$ \\
\hline$B R C A 2$ & $\begin{array}{l}\text { OMIM Cancer, 24429628, 28600339, 29489754, 29625052, 26556299, } \\
28199314,2658044\end{array}$ \\
\hline BRCA3 & OMIM Cancer \\
\hline BRCC3 & 25426837 \\
\hline$B R I P 1$ & $\begin{array}{l}\text { OMIM Cancer, 24429628, 28600339, 29489754, 29625052, 26556299, } \\
2819931\end{array}$ \\
\hline BTG1 & 25426837 \\
\hline BTG2 & 25426837 \\
\hline BTK & 26556299 \\
\hline$B U B 1$ & OMIM Cancer \\
\hline$B U B 1 B$ & OMIM Cancer, 24429628, 29489754, 29625052, 28199314 \\
\hline
\end{tabular}




\begin{tabular}{|c|c|}
\hline CACNA1S & OMIM Cancer \\
\hline CALR & OMIM Myeloid Leukemia \\
\hline CARD11 & 25426837,26556299 \\
\hline CASP10 & OMIM Cancer \\
\hline CASP8 & OMIM Cancer, 26556299 \\
\hline$C B F B$ & OMIM Myeloid Leukemia \\
\hline$C B L$ & $\begin{array}{l}\text { 24429628, 27276561, 29489754, 29625052, 25426837, OMIM Myeloid } \\
\text { Leukemia, 26556299, } 2658044\end{array}$ \\
\hline$C B L B$ & 27276561,25426837 \\
\hline CBLC & 27276561 \\
\hline$C B P$ & 29489754 \\
\hline CCND1 & OMIM Cancer, OMIM Myeloid Leukemia \\
\hline CCND2 & 26556299 \\
\hline CCND3 & 25426837 \\
\hline$C D 101$ & 27276561 \\
\hline CD58 & 25426837 \\
\hline$C D 70$ & 25426837 \\
\hline$C D 79 A$ & 25426837 \\
\hline$C D 79 B$ & 25426837,26556299 \\
\hline CD82 & OMIM Cancer \\
\hline$C D C 73$ & OMIM Cancer, 24429628, 29489754, 29625052, 26556299, 26580448 \\
\hline $\mathrm{CDH} 1$ & $\begin{array}{l}\text { OMIM Cancer, 24429628, 27276561, 29489754, 29625052, 26556299, } \\
28199314,2658044\end{array}$ \\
\hline CDK4 & $\begin{array}{l}\text { OMIM Cancer, 24429628, 29489754, 29625052, 26556299, 28199314, } \\
2658044\end{array}$ \\
\hline CDKN1B & 24429628, 27276561, 29489754, 29625052, 26556299, 28199314 \\
\hline
\end{tabular}




\begin{tabular}{|c|c|}
\hline CDKN1C & $29489754,29625052,26580448$ \\
\hline$C D K N 2 A$ & $\begin{array}{l}\text { OMIM Cancer, 24429628, 27276561, 29489754, 29625052, 25426837, } \\
26556299,28199314,2658044\end{array}$ \\
\hline$C D K N 2 B$ & 27276561,25426837 \\
\hline CEBPA & $\begin{array}{l}\text { 24429628, 27276561, 28600339, 29489754, 29625052, 25426837, OMIM } \\
\text { Myeloid Leukemia, } 2658044\end{array}$ \\
\hline CHD2 & 25426837 \\
\hline CHEK2 & OMIM Cancer, 24429628, 29489754, 29625052, 26556299, 28199314 \\
\hline CHGA & 27276561 \\
\hline CHIC2 & OMIM Myeloid Leukemia \\
\hline CHRNA3 & OMIM Cancer \\
\hline CHRNA5 & OMIM Cancer \\
\hline CMM & OMIM Cancer \\
\hline CNOT3 & 25426837 \\
\hline COL7A1 & $24429628,29489754,29625052$ \\
\hline CREBBP & 27276561, 29489754, 25426837, 26556299 \\
\hline CRLF2 & 25426837 \\
\hline CRTC1 & OMIM Cancer \\
\hline CSF1R & $27276561,25426837,26556299$ \\
\hline CSF2 & 27276561 \\
\hline CSF3R & 25426837 \\
\hline CTC1 & 28600339 \\
\hline CTCF & 25426837,26556299 \\
\hline CTHRC1 & OMIM Cancer \\
\hline CTLA4 & 26556299 \\
\hline CTNNA1 & 27276561,28199314 \\
\hline
\end{tabular}




\begin{tabular}{|c|c|}
\hline CTNNB1 & OMIM Cancer, 26556299 \\
\hline CTR9 & 29625052 \\
\hline CUL3 & 26556299 \\
\hline CUX1 & 27276561,25426837 \\
\hline CXCR4 & OMIM Myeloid Leukemia \\
\hline$C Y L D$ & $24429628,29489754,29625052$ \\
\hline CYP2A6 & OMIM Cancer \\
\hline DARS & OMIM Myeloid Leukemia \\
\hline$D C C$ & OMIM Cancer \\
\hline DDB2 & $24429628,29489754,29625052$ \\
\hline DDR2 & 26556299 \\
\hline$D D X 18$ & 27276561 \\
\hline$D D X 3 X$ & 25426837 \\
\hline$D D X 41$ & 28600339, OMIM Myeloid Leukemia \\
\hline DEC1 & OMIM Cancer \\
\hline DEK & OMIM Myeloid Leukemia \\
\hline DHCR7 & 29489754 \\
\hline DICER1 & $\begin{array}{l}\text { OMIM Cancer, 24429628, 29489754, 29625052, 26556299, 28199314, } \\
26580448\end{array}$ \\
\hline DIRC2 & OMIM Cancer \\
\hline DIS3 & 25426837 \\
\hline DIS3L2 & $24429628,29489754,29625052,28199314$ \\
\hline DKC1 & $24429628,28600339,29489754,29625052$ \\
\hline$D L C 1$ & OMIM Cancer \\
\hline DLEC1 & OMIM Cancer \\
\hline DNMT1 & 27276561,26556299 \\
\hline
\end{tabular}




\begin{tabular}{|c|c|}
\hline DNMT3A & $27276561,25426837,26556299$ \\
\hline DNMT3B & 26556299 \\
\hline DOCK8 & $24429628,29489754,29625052$ \\
\hline DROSHA & 29625052 \\
\hline EBF1 & 25426837 \\
\hline$E E D$ & 25426837 \\
\hline$E G F R$ & $\begin{array}{l}\text { OMIM Cancer, 24429628, 27276561, 29489754, 29625052, 26556299, } \\
2819931\end{array}$ \\
\hline EGR2 & OMIM Myeloid Leukemia \\
\hline EHBP1 & OMIM Cancer \\
\hline ELAC2 & OMIM Cancer \\
\hline ELANE & $24429628,28600339,29489754,29625052$ \\
\hline ELF1 & 27276561 \\
\hline EP300 & OMIM Cancer, 27276561, 29489754, 25426837, 26556299 \\
\hline EPCAM & OMIM Cancer, 29489754, 29625052, 26556299, 28199314, 26580448 \\
\hline EPHB2 & OMIM Cancer \\
\hline$E R B B 2$ & OMIM Cancer \\
\hline ERBB3 & 26556299 \\
\hline ERBB4 & 26556299 \\
\hline ERCC1 & 29489754,29625052 \\
\hline ERCC2 & 24429628, 29489754, 29625052, 26556299, 28199314 \\
\hline ERCC3 & 24429628, 29489754, 29625052, 26556299, 28199314 \\
\hline ERCC4 & $24429628,28600339,29489754,29625052,26556299,28199314$ \\
\hline ERCC5 & 24429628, 29489754, 29625052, 26556299, 28199314 \\
\hline ERCC6 & OMIM Cancer \\
\hline$E R G$ & 27276561 \\
\hline
\end{tabular}




\begin{tabular}{|c|c|}
\hline ESR1 & OMIM Cancer, 26556299 \\
\hline ETV6 & $\begin{array}{l}\text { 27276561, 28600339, 29625052, 25426837, OMIM Myeloid Leukemia, } \\
26556299\end{array}$ \\
\hline EXT1 & $24429628,29489754,29625052,28199314$ \\
\hline EXT2 & 24429628, 29489754, 29625052, 28199314 \\
\hline$E Z H 2$ & $27276561,25426837,26556299$ \\
\hline$E Z R$ & 25426837 \\
\hline$F A H$ & $24429628,29489754,29625052$ \\
\hline FAM126A & OMIM Myeloid Leukemia \\
\hline FAM175A & 26556299,28199314 \\
\hline FAM175B & 27276561 \\
\hline FAM46C & 25426837 \\
\hline FANCA & $24429628,28600339,29489754,29625052,26556299,28199314$ \\
\hline FANCB & 28600339,28199314 \\
\hline FANCC & $24429628,28600339,29489754,29625052,26556299,28199314$ \\
\hline FANCD1 & 28600339 \\
\hline FANCD2 & $28600339,29489754,29625052,28199314$ \\
\hline FANCDE & 28600339 \\
\hline FANCE & $29489754,29625052,28199314$ \\
\hline FANCF & 28600339, 29489754, 29625052, 28199314 \\
\hline FANCG & $24429628,28600339,29489754,29625052,28199314$ \\
\hline $\mathrm{FANCl}$ & 28600339, 29489754, 29625052, 28199314 \\
\hline FANCJ & 28600339 \\
\hline FANCL & $28600339,29489754,29625052,28199314$ \\
\hline FANCM & 28600339, 29489754, 29625052, 28199314 \\
\hline FANCN & 28600339 \\
\hline
\end{tabular}




\begin{tabular}{|c|c|}
\hline FANCO & 28600339 \\
\hline FANCP & 28600339 \\
\hline FANCQ & 28600339 \\
\hline FANCR & 28600339 \\
\hline FANCS & 28600339 \\
\hline FANCT & 28600339 \\
\hline FANCU & 28600339 \\
\hline FANCV & 28600339 \\
\hline FAND2 & 29489754 \\
\hline FAS & OMIM Cancer, 24429628, 25426837 \\
\hline FASLG & OMIM Cancer \\
\hline FBX011 & 25426837 \\
\hline FBXW7 & 27276561,25426837 \\
\hline FGF23 & OMIM Cancer \\
\hline FGF3 & 26556299 \\
\hline FGFR1 & 26556299 \\
\hline FGFR1OP & OMIM Myeloid Leukemia \\
\hline FGFR2 & OMIM Cancer, 29489754, 26556299 \\
\hline FGFR3 & OMIM Cancer, 29489754, 26556299 \\
\hline FGFR4 & OMIM Cancer \\
\hline$F H$ & $\begin{array}{l}\text { OMIM Cancer, 24429628, 29489754, 29625052, 26556299, 28199314, } \\
2658044\end{array}$ \\
\hline FLCN & OMIM Cancer, 24429628, 29489754, 29625052, 26556299 \\
\hline FLT3 & 27276561, 25426837, OMIM Myeloid Leukemia \\
\hline FLT4 & 26556299 \\
\hline FMR1 & 29489754 \\
\hline
\end{tabular}




\begin{tabular}{|c|c|}
\hline FOXE1 & OMIM Cancer \\
\hline FOXL2 & 26556299 \\
\hline FOXP1 & 25426837,26556299 \\
\hline$F Y N$ & 25426837 \\
\hline GALNT12 & OMIM Cancer \\
\hline GALNT3 & OMIM Cancer \\
\hline GATA1 & 27276561, 28600339, 25426837, OMIM Myeloid Leukemia, 26556299 \\
\hline GATA2 & $\begin{array}{l}\text { 24429628, 27276561, 28600339, 29489754, 29625052, 25426837, OMIM } \\
\text { Myeloid Leukemia, 26556299, 28199314, } 2658044\end{array}$ \\
\hline GATA3 & 25426837,26556299 \\
\hline GBA & $24429628,29489754,29625052$ \\
\hline GJB2 & $24429628,29489754,29625052$ \\
\hline GJC2 & OMIM Myeloid Leukemia \\
\hline GLI3 & 29489754 \\
\hline GNA11 & 26556299 \\
\hline GNA13 & 25426837 \\
\hline GNAQ & 26556299 \\
\hline GNAS & $27276561,25426837,26556299$ \\
\hline GNAS1 & 29489754 \\
\hline GNB1 & 25426837, OMIM Myeloid Leukemia \\
\hline GPC3 & OMIM Cancer, 24429628, 29489754, 29625052 \\
\hline GREM1 & 26556299 \\
\hline GRIN2A & 26556299 \\
\hline$H 19$ & OMIM Cancer, 29489754 \\
\hline HABP2 & OMIM Cancer \\
\hline HAX1 & 28600339 \\
\hline
\end{tabular}




\begin{tabular}{|c|c|}
\hline HFE & $24429628,29489754,29625052$ \\
\hline HGF & 26556299 \\
\hline HIKESHI & OMIM Myeloid Leukemia \\
\hline HIP1 & OMIM Cancer \\
\hline HIPK2 & 27276561 \\
\hline HIST1H1B & 25426837 \\
\hline HIST1H1C & 25426837 \\
\hline HIST1H1D & 25426837 \\
\hline HIST1H1E & 25426837 \\
\hline HIST1H3B & 25426837 \\
\hline HLAVA & 25426837 \\
\hline$H M B S$ & $24429628,29489754,29625052$ \\
\hline HMGA2 & 27276561 \\
\hline HMMR & OMIM Cancer \\
\hline HNF1A & OMIM Cancer, 29625052, 26556299 \\
\hline HNF1B & OMIM Cancer \\
\hline HOXB13 & 28199314 \\
\hline HPCX & OMIM Cancer \\
\hline HRAS & $\begin{array}{l}\text { OMIM Cancer, 24429628, 27276561, 29489754, 29625052, 26556299, } \\
2658044\end{array}$ \\
\hline HSPD1 & OMIM Myeloid Leukemia \\
\hline ID3 & 25426837 \\
\hline$I D H 1$ & $27276561,25426837,26556299$ \\
\hline$I D H 2$ & $27276561,25426837,26556299$ \\
\hline IFNGR1 & 26556299 \\
\hline IGF1 & 26556299 \\
\hline
\end{tabular}




\begin{tabular}{|c|c|}
\hline IGF1R & 26556299 \\
\hline IGF2R & OMIM Cancer \\
\hline$I K B K B$ & 25426837 \\
\hline IKBKG & OMIM Cancer \\
\hline IKZF1 & 27276561,25426837 \\
\hline IKZF2 & 25426837 \\
\hline IKZF3 & 25426837 \\
\hline IL1B & OMIM Cancer \\
\hline IL1RN & OMIM Cancer \\
\hline IL7R & 25426837,26556299 \\
\hline ING1 & OMIM Cancer \\
\hline INSR & 26556299 \\
\hline INTS12 & 25426837 \\
\hline INVS & 27276561 \\
\hline IRAK4 & OMIM Cancer \\
\hline IRF1 & OMIM Cancer, 27276561, OMIM Myeloid Leukemia \\
\hline IRF4 & 25426837 \\
\hline IRF8 & 25426837 \\
\hline ITK & $24429628,29489754,29625052$ \\
\hline JAK1 & 25426837 \\
\hline JAK2 & 27276561, 25426837, OMIM Myeloid Leukemia, 26556299 \\
\hline JAK3 & $27276561,25426837,26556299$ \\
\hline JARID2 & 25426837 \\
\hline JMJD1C & 29625052 \\
\hline KCNQ1OT1 & 29489754 \\
\hline KDM2B & 27276561 \\
\hline
\end{tabular}




\begin{tabular}{|c|c|}
\hline KDM5A & 27276561 \\
\hline KDM5C & 26556299 \\
\hline KDM6A & $27276561,25426837,26556299$ \\
\hline$K D R$ & 26556299 \\
\hline KDSR & OMIM Myeloid Leukemia \\
\hline KIT & $\begin{array}{l}\text { OMIM Cancer, 24429628, 27276561, 29489754, 29625052, 25426837, OMIM } \\
\text { Myeloid Leukemia, 26556299, } 2819931\end{array}$ \\
\hline$K L$ & OMIM Cancer \\
\hline KLF6 & OMIM Cancer \\
\hline KLHL6 & 25426837 \\
\hline$K L L N$ & 28199314 \\
\hline KMT2A & 27276561, OMIM Myeloid Leukemia, 26556299 \\
\hline KMT2C & 27276561 \\
\hline KMT2D & 27276561,26556299 \\
\hline KMT2E & 27276561 \\
\hline KRAS & $\begin{array}{l}\text { OMIM Cancer, 27276561, 29489754, 29625052, 25426837, OMIM Myeloid } \\
\text { Leukemia, 26556299, } 2658044\end{array}$ \\
\hline L2HGDH & 29489754 \\
\hline LCORL & 27276561 \\
\hline LEF1 & OMIM Cancer, 25426837 \\
\hline LIG4 & OMIM Myeloid Leukemia \\
\hline LILRA3 & 27276561 \\
\hline LMO1 & 29625052, OMIM Myeloid Leukemia \\
\hline LMO2 & OMIM Myeloid Leukemia \\
\hline$L P P$ & OMIM Myeloid Leukemia \\
\hline LRRK2 & 25426837 \\
\hline
\end{tabular}




\begin{tabular}{|c|c|}
\hline$L T B$ & 25426837 \\
\hline LUC7L2 & 25426837 \\
\hline LYL1 & OMIM Myeloid Leukemia \\
\hline LZTR1 & 29489754 \\
\hline LZTS1 & OMIM Cancer \\
\hline MAD1L1 & OMIM Cancer \\
\hline$M A L T 1$ & 25426837 \\
\hline MAML2 & OMIM Cancer \\
\hline MAP2K1 & $29489754,29625052,25426837,26556299,26580448$ \\
\hline MAP2K2 & 29489754, 29625052, 26556299, 26580448 \\
\hline MAP2K5 & 27276561 \\
\hline MAP3K1 & 26556299 \\
\hline MAP3K14 & 25426837 \\
\hline MAPЗK8 & OMIM Cancer \\
\hline MAX & $24429628,29489754,29625052,26556299,28199314,26580448$ \\
\hline MC1R & OMIM Cancer \\
\hline$M C C$ & OMIM Cancer \\
\hline MDM2 & OMIM Cancer \\
\hline MECOM & 27276561 \\
\hline MED12 & 25426837,26556299 \\
\hline MEF2B & 25426837 \\
\hline MEN1 & $\begin{array}{l}\text { OMIM Cancer, 24429628, 29489754, 29625052, 26556299, 28199314, } \\
2658044\end{array}$ \\
\hline MET & $\begin{array}{l}\text { OMIM Cancer, 24429628, 27276561, 29489754, 29625052, 26556299, } \\
2819931\end{array}$ \\
\hline MFHAS1 & OMIM Cancer \\
\hline
\end{tabular}




\begin{tabular}{|c|c|}
\hline MHS2 & OMIM Cancer \\
\hline MHS4 & OMIM Cancer \\
\hline MHS6 & OMIM Cancer \\
\hline MINPP1 & OMIM Cancer \\
\hline MITF & OMIM Cancer, 29489754, 29625052, 26556299, 28199314 \\
\hline MKL1 & OMIM Myeloid Leukemia \\
\hline$M L F 1$ & OMIM Myeloid Leukemia \\
\hline MLH1 & $\begin{array}{l}\text { OMIM Cancer, 24429628, 28600339, 29489754, 29625052, 26556299, } \\
28199314,2658044\end{array}$ \\
\hline MLH3 & OMIM Cancer \\
\hline$M L L$ & 25426837 \\
\hline MLL2 & 25426837 \\
\hline MLLT10 & OMIM Myeloid Leukemia \\
\hline MMD2 & 27276561 \\
\hline MMR & 29489754 \\
\hline MN1 & 27276561 \\
\hline$M P L$ & $\begin{array}{l}\text { 27276561, 29625052, 25426837, OMIM Myeloid Leukemia, 26556299, } \\
28199314\end{array}$ \\
\hline MPO & OMIM Cancer, OMIM Myeloid Leukemia \\
\hline$M P Z$ & OMIM Myeloid Leukemia \\
\hline MRE11A & $29489754,26556299,28199314$ \\
\hline MSH2 & $\begin{array}{l}\text { OMIM Cancer, 24429628, 28600339, 29489754, 29625052, 26556299, } \\
28199314,2658044\end{array}$ \\
\hline $\mathrm{MSH3}$ & OMIM Cancer \\
\hline MSH6 & $\begin{array}{l}\text { OMIM Cancer, 24429628, 28600339, 29489754, 29625052, 26556299, } \\
28199314,2658044\end{array}$ \\
\hline
\end{tabular}




\begin{tabular}{|c|c|}
\hline$M S M B$ & OMIM Cancer \\
\hline MSR1 & OMIM Cancer \\
\hline MST1R & OMIM Cancer \\
\hline MTAP & OMIM Cancer, 24429628, 27276561, 29489754, 29625052 \\
\hline MUTYH & OMIM Cancer, 24429628, 29489754, 29625052, 26556299, 28199314 \\
\hline$M X I 1$ & OMIM Cancer \\
\hline MXRA5 & 25426837 \\
\hline$M Y B$ & OMIM Myeloid Leukemia \\
\hline$M Y C$ & 27276561 \\
\hline MYCN & 26556299 \\
\hline MYD88 & 25426837,26556299 \\
\hline NAF1 & 28600339 \\
\hline$N B N$ & $\begin{array}{l}\text { 24429628, 29489754, 29625052, OMIM Myeloid Leukemia, 26556299, } \\
28199314\end{array}$ \\
\hline NBS1 & 29489754 \\
\hline NDUFA13 & OMIM Cancer \\
\hline NEK1 & 28199314 \\
\hline NF1 & $\begin{array}{l}\text { 24429628, 27276561, 29489754, 29625052, OMIM Myeloid Leukemia, } \\
\text { 26556299, 28199314, } 2658044\end{array}$ \\
\hline NF2 & $24429628,29489754,29625052,26556299,28199314,26580448$ \\
\hline NFIX1 & 29489754 \\
\hline NHP2 & $28600339,29489754,29625052$ \\
\hline$N K X 2-1$ & OMIM Cancer, 26556299 \\
\hline NLRP1 & 27276561 \\
\hline NOLA2 & 28600339 \\
\hline NOLA3 & 28600339 \\
\hline
\end{tabular}




\begin{tabular}{|c|c|}
\hline NOP10 & $28600339,29489754,29625052$ \\
\hline $\mathrm{NOTCH} 1$ & $27276561,25426837,26556299$ \\
\hline $\mathrm{NOTCH} 2$ & 25426837,26556299 \\
\hline $\mathrm{NOTCH} 3$ & 26556299 \\
\hline NPM1 & 27276561, 25426837, OMIM Myeloid Leukemia \\
\hline NQO1 & OMIM Cancer, OMIM Myeloid Leukemia \\
\hline NQO2 & OMIM Cancer \\
\hline NR5A1 & 27276561 \\
\hline NRAS & $\begin{array}{l}\text { OMIM Cancer, 27276561, 29489754, 29625052, 25426837, 26556299, } \\
2658044\end{array}$ \\
\hline NRDC & 27276561 \\
\hline NSD1 & 27276561, 29489754, OMIM Myeloid Leukemia, 26556299 \\
\hline NTHL1 & 29625052 \\
\hline NTRK1 & OMIM Cancer, 29489754, 26556299, 28199314 \\
\hline NTRK2 & 26556299 \\
\hline NUMA1 & OMIM Myeloid Leukemia \\
\hline NUP214 & OMIM Myeloid Leukemia \\
\hline NUP98 & 27276561 \\
\hline OCA2 & 27276561 \\
\hline OGG1 & OMIM Cancer \\
\hline OPCML & OMIM Cancer \\
\hline P2RY8 & 25426837 \\
\hline PALB2 & $\begin{array}{l}\text { OMIM Cancer, 24429628, 28600339, 29489754, 29625052, 26556299, } \\
28199314,2658044\end{array}$ \\
\hline PALLD & OMIM Cancer \\
\hline PAPD5 & 25426837 \\
\hline
\end{tabular}




\begin{tabular}{|c|c|}
\hline PARK2 & OMIM Cancer, 26556299 \\
\hline PARN & 28600339 \\
\hline PAX5 & $\begin{array}{l}\text { 29489754, 29625052, 25426837, OMIM Myeloid Leukemia, 26556299, } \\
26580448\end{array}$ \\
\hline PAX5 & $\begin{array}{l}\text { 29489754, 29625052, 25426837, OMIM Myeloid Leukemia, 26556299, } \\
26580448\end{array}$ \\
\hline$P B X 1$ & OMIM Myeloid Leukemia \\
\hline PCAP & OMIM Cancer \\
\hline PDCD1 & 26556299 \\
\hline PDGFRA & OMIM Cancer, 24429628, 27276561, 29489754, 29625052, 26556299 \\
\hline PDGFRB & OMIM Myeloid Leukemia, 26556299 \\
\hline PDGFRL & OMIM Cancer \\
\hline PDS5B & 25426837 \\
\hline PDSS2 & 25426837 \\
\hline PHB & OMIM Cancer \\
\hline PHF12 & 27276561 \\
\hline PHF6 & 27276561,25426837 \\
\hline PHOX2B & $24429628,29489754,29625052,26556299,28199314,26580448$ \\
\hline PICALM & OMIM Myeloid Leukemia \\
\hline PIKЗCA & OMIM Cancer, 25426837, 26556299 \\
\hline PIKЗCD & 26556299 \\
\hline PIK3R1 & 26556299 \\
\hline PIK3R2 & 26556299 \\
\hline PKP3 & 27276561 \\
\hline PLA2G2A & OMIM Cancer \\
\hline$P M L$ & OMIM Myeloid Leukemia \\
\hline
\end{tabular}




\begin{tabular}{|c|c|}
\hline PMP22 & OMIM Myeloid Leukemia \\
\hline PMS1 & $29625052,26556299,28199314$ \\
\hline PMS2 & $\begin{array}{l}\text { OMIM Cancer, 24429628, 29489754, 29625052, 26556299, 28199314, } \\
2658044\end{array}$ \\
\hline POLD1 & OMIM Cancer, 24429628, 29489754, 29625052, 28199314 \\
\hline POLE & OMIM Cancer, 24429628, 29489754, 29625052, 26556299, 2819931 \\
\hline POLH & $24429628,29489754,29625052$ \\
\hline POLR1C & OMIM Myeloid Leukemia \\
\hline POLR3A & OMIM Myeloid Leukemia \\
\hline POLR3B & OMIM Myeloid Leukemia \\
\hline POT1 & OMIM Cancer, 29625052, 25426837 \\
\hline POU2AF1 & 25426837 \\
\hline POU2F2 & 25426837 \\
\hline POU6F2 & OMIM Cancer \\
\hline$P P M 1 D$ & OMIM Cancer \\
\hline PPP2R1B & OMIM Cancer \\
\hline$P R C C$ & OMIM Cancer \\
\hline PRDM1 & 25426837 \\
\hline PRDM9 & 29625052 \\
\hline PRDX2 & 27276561 \\
\hline PRF1 & 29625052,28199314 \\
\hline PRKAR1A & $\begin{array}{l}\text { OMIM Cancer, 24429628, 29489754, 29625052, 26556299, 28199314, } \\
26580448\end{array}$ \\
\hline PRKCA & OMIM Cancer \\
\hline PRPF4OB & 27276561,25426837 \\
\hline PRPF8 & 25426837 \\
\hline
\end{tabular}




\begin{tabular}{|c|c|}
\hline PRSS1 & $24429628,29489754,29625052$ \\
\hline $\mathrm{PTCH} 1$ & $\begin{array}{l}\text { OMIM Cancer, 24429628, 29489754, 29625052, 26556299, 28199314, } \\
2658044\end{array}$ \\
\hline PTCH2 & OMIM Cancer \\
\hline PTEN & $\begin{array}{l}\text { OMIM Cancer, 24429628, 27276561, 29489754, 29625052, 25426837, } \\
26556299,2658044\end{array}$ \\
\hline PTHLH & OMIM Cancer \\
\hline PTPN1 & 25426837 \\
\hline PTPN11 & $\begin{array}{l}\text { 24429628, 27276561, 29489754, 29625052, 25426837, OMIM Myeloid } \\
\text { Leukemia, 26556299, } 2658044\end{array}$ \\
\hline PTPN12 & OMIM Cancer \\
\hline PTPRJ & OMIM Cancer \\
\hline PYCR2 & OMIM Myeloid Leukemia \\
\hline$R A D 21$ & 27276561,25426837 \\
\hline RAD50 & $27276561,26556299,28199314$ \\
\hline RAD51 & OMIM Cancer, 28600339, 29489754, 26556299 \\
\hline$R A D 51 B$ & 26556299 \\
\hline$R A D 51 C$ & $\begin{array}{l}\text { OMIM Cancer, 24429628, 28600339, 29489754, 29625052, 26556299, } \\
2819931\end{array}$ \\
\hline RAD51D & OMIM Cancer, 24429628, 29489754, 29625052, 26556299, 28199314 \\
\hline$R A D 54 B$ & OMIM Cancer \\
\hline$R A D 54 L$ & OMIM Cancer, 28199314 \\
\hline RAF1 & $29489754,29625052,26556299,26580448$ \\
\hline RAG1 & OMIM Cancer \\
\hline RAG2 & OMIM Cancer \\
\hline RAP1GDS1 & OMIM Myeloid Leukemia \\
\hline
\end{tabular}




\begin{tabular}{|c|c|}
\hline$R A R A$ & OMIM Myeloid Leukemia \\
\hline$R A R S$ & OMIM Myeloid Leukemia \\
\hline RASA1 & OMIM Cancer, 26556299 \\
\hline RASSF1 & OMIM Cancer \\
\hline$R B 1$ & $\begin{array}{l}\text { OMIM Cancer, 24429628, 27276561, 29489754, 29625052, 26556299, } \\
28199314,2658044\end{array}$ \\
\hline$R B 1 C C 1$ & OMIM Cancer \\
\hline RBBP4 & 25426837 \\
\hline RBBP8 & OMIM Cancer \\
\hline$R B M 10$ & 26556299 \\
\hline RBM15 & OMIM Myeloid Leukemia \\
\hline$R E C Q L$ & 29625052 \\
\hline RECQL3 & 29489754 \\
\hline RECQL4 & $24429628,29489754,29625052,26556299,28199314$ \\
\hline REST & OMIM Cancer \\
\hline$R E T$ & $\begin{array}{l}\text { OMIM Cancer, 24429628, 29489754, 29625052, 26556299, 28199314, } \\
2658044\end{array}$ \\
\hline REV7 & 28600339 \\
\hline RHBDF2 & OMIM Cancer, 24429628, 29489754, 29625052 \\
\hline RHOA & 25426837 \\
\hline RINT1 & 27276561 \\
\hline RIT1 & 25426837,26556299 \\
\hline$R M R P$ & $24429628,29489754,29625052$ \\
\hline RNASEL & OMIM Cancer \\
\hline RNF139 & OMIM Cancer \\
\hline RNF43 & OMIM Cancer \\
\hline
\end{tabular}




\begin{tabular}{|c|c|}
\hline$R N F 6$ & OMIM Cancer \\
\hline$R O R C$ & 27276561 \\
\hline RPL10 & 25426837 \\
\hline RPL11 & 28600339 \\
\hline$R P L 15$ & 28600339 \\
\hline RPL23 & 28600339 \\
\hline RPL26 & 28600339 \\
\hline RPL27 & 28600339 \\
\hline RPL31 & 28600339 \\
\hline RPL35A & 28600339 \\
\hline RPL36 & 28600339 \\
\hline RPL5 & 28600339,25426837 \\
\hline RPS10 & 28600339 \\
\hline RPS15 & 28600339,25426837 \\
\hline$R P S 17$ & 28600339 \\
\hline RPS19 & 28600339 \\
\hline RPS2 & 25426837 \\
\hline RPS24 & 28600339 \\
\hline RPS26 & 28600339 \\
\hline RPS27 & 28600339 \\
\hline RPS27A & 28600339 \\
\hline RPS28 & 28600339 \\
\hline RPS29 & 28600339 \\
\hline RPS7 & 28600339 \\
\hline RRAS2 & OMIM Cancer \\
\hline RSPO1 & OMIM Cancer \\
\hline
\end{tabular}




\begin{tabular}{|c|c|}
\hline RTEL1 & 28600339,28199314 \\
\hline$R \cup N X 1$ & $\begin{array}{l}\text { OMIM Cancer, 24429628, 27276561, 28600339, 29489754, 29625052, } \\
\text { 25426837, OMIM Myeloid Leukemia, 26556299, } 2658044\end{array}$ \\
\hline$R U N X 1 T 1$ & 27276561 \\
\hline$R Y R 1$ & OMIM Cancer \\
\hline SAMD9 & OMIM Cancer, 28600339 \\
\hline SAMD9L & 28600339 \\
\hline SBDS & $24429628,28600339,29489754,29625052,28199314$ \\
\hline$S D H A$ & $24429628,29489754,29625052,26556299,28199314,26580448$ \\
\hline SDHAF2 & $24429628,29489754,29625052,26556299,26580448$ \\
\hline$S D H B$ & $\begin{array}{l}\text { OMIM Cancer, 24429628, 29489754, 29625052, 26556299, 28199314, } \\
2658044\end{array}$ \\
\hline$S D H C$ & $\begin{array}{l}\text { OMIM Cancer, 24429628, 29489754, 29625052, 26556299, 28199314, } \\
2658044\end{array}$ \\
\hline$S D H D$ & $\begin{array}{l}\text { OMIM Cancer, 24429628, 29489754, 29625052, 26556299, 28199314, } \\
2658044\end{array}$ \\
\hline SEPT9 & OMIM Myeloid Leukemia, OMIM Cancer \\
\hline SERPINA1 & $24429628,29489754,29625052$ \\
\hline SETBP1 & 29489754,29625052 \\
\hline SF1 & 27276561 \\
\hline SF3A1 & 27276561,25426837 \\
\hline SF3B1 & 27276561, 25426837, OMIM Myeloid Leukemia \\
\hline SFRS2 & 25426837 \\
\hline SGK1 & 25426837 \\
\hline SH2B3 & 27276561, 29489754, 29625052, OMIM Myeloid Leukemia \\
\hline SH2D1A & $24429628,29489754,29625052,26556299$ \\
\hline
\end{tabular}




\begin{tabular}{|c|c|}
\hline SH3GL1 & OMIM Myeloid Leukemia \\
\hline SHOC2 & $29489754,29625052,26580448$ \\
\hline SLC22A18 & OMIM Cancer \\
\hline SLC25A13 & $24429628,29489754,29625052$ \\
\hline SLX4 & $28600339,29489754,28199314$ \\
\hline SMAD3 & 26556299 \\
\hline$S M A D 4$ & $\begin{array}{l}\text { OMIM Cancer, 24429628, 29489754, 29625052, 26556299, 28199314, } \\
2658044\end{array}$ \\
\hline SMAD7 & OMIM Cancer \\
\hline SMARCA2 & 29489754 \\
\hline SMARCA4 & OMIM Cancer, 24429628, 29489754, 29625052, 26556299, 26580448 \\
\hline SMARCB1 & OMIM Cancer, 24429628, 29489754, 29625052, 26556299, 26580448 \\
\hline SMARCE1 & $24429628,29489754,29625052$ \\
\hline SMC1A & 25426837 \\
\hline SMC3 & 25426837 \\
\hline$S M O$ & OMIM Cancer \\
\hline SOCS1 & 27276561,25426837 \\
\hline SOS1 & $24429628,29489754,29625052,26580448$ \\
\hline SOX17 & 26556299 \\
\hline SOX2 & 26556299 \\
\hline SOX9 & 26556299 \\
\hline SPI1 & 27276561 \\
\hline SPRTN & 29625052 \\
\hline SPRY4 & 25426837 \\
\hline$S R C$ & OMIM Cancer \\
\hline SRGAP1 & OMIM Cancer \\
\hline
\end{tabular}




\begin{tabular}{|c|c|}
\hline SRP72 & 28600339 \\
\hline SRPK2 & 27276561 \\
\hline SRSF2 & 27276561 \\
\hline$S R Y$ & $24429628,29489754,29625052$ \\
\hline ST11 & OMIM Cancer \\
\hline ST3 & OMIM Cancer \\
\hline STAG1 & 25426837 \\
\hline STAG2 & 27276561,25426837 \\
\hline STAT3 & $24429628,29489754,29625052,25426837$ \\
\hline STAT5A & 25426837 \\
\hline STAT5B & 25426837, OMIM Myeloid Leukemia \\
\hline STAT6 & 25426837 \\
\hline STK11 & $\begin{array}{l}\text { OMIM Cancer, 24429628, 29489754, 29625052, 26556299, 28199314, } \\
2658044\end{array}$ \\
\hline STK17B & 27276561 \\
\hline STN1 & 28600339 \\
\hline SUFU & $24429628,29489754,29625052,26556299,28199314,26580448$ \\
\hline SUZ12 & 25426837 \\
\hline SWAP70 & 25426837 \\
\hline TAL1 & OMIM Myeloid Leukemia \\
\hline$T$ TAL2 & OMIM Myeloid Leukemia \\
\hline$T B L 1 X R 1$ & 25426837 \\
\hline$T B X 3$ & 26556299 \\
\hline TCAB1 & 28600339 \\
\hline TCF3 & 25426837 \\
\hline TCF4 & 27276561 \\
\hline
\end{tabular}




\begin{tabular}{|c|c|}
\hline TCL1A & OMIM Myeloid Leukemia \\
\hline TCL1B & OMIM Myeloid Leukemia \\
\hline TCL4 & OMIM Myeloid Leukemia \\
\hline TERC & 28600339 \\
\hline TERT & $\begin{array}{l}\text { OMIM Cancer, 24429628, 28600339, 29489754, 29625052, OMIM Myeloid } \\
\text { Leukemia, 26556299, } 2819931\end{array}$ \\
\hline TET1 & 27276561,25426837 \\
\hline TET2 & 27276561, 25426837, OMIM Myeloid Leukemia \\
\hline TFAP2A & 29489754 \\
\hline TFE3 & OMIM Cancer \\
\hline TGCT1 & OMIM Cancer \\
\hline TGFBR1 & $24429628,29489754,29625052,26556299$ \\
\hline TGFBR2 & OMIM Cancer, 26556299 \\
\hline TINF2 & 28600339 \\
\hline TIRAP & OMIM Cancer \\
\hline TLR2 & OMIM Cancer \\
\hline TMEM127 & $24429628,29489754,29625052,26556299,26580448$ \\
\hline TMEMЗОA & 25426837 \\
\hline TNF & 25426837 \\
\hline TNFAIP3 & 25426837 \\
\hline $\begin{array}{l}\text { TNFRSF10 } \\
B\end{array}$ & OMIM Cancer \\
\hline TNFRSF14 & 25426837 \\
\hline TNFRSF6 & 29489754,29625052 \\
\hline TP53 & $\begin{array}{l}\text { OMIM Cancer, 24429628, 27276561, 28600339, 29489754, 29625052, } \\
25426837,26556299,28199314,2658044\end{array}$ \\
\hline
\end{tabular}




\begin{tabular}{|c|c|}
\hline TP63 & 26556299 \\
\hline TPMS2 & 28600339 \\
\hline TPP1 & 28600339 \\
\hline TRAF3 & 25426837 \\
\hline TRIM37 & 24429628, 29489754 \\
\hline TRIM37 & 24429628,29489754 \\
\hline TSC1 & $24429628,29489754,29625052,26556299,28199314,26580448$ \\
\hline TSC2 & $24429628,29489754,29625052,26556299,28199314,26580448$ \\
\hline TSG101 & OMIM Cancer \\
\hline TSHR & OMIM Cancer, 29625052, 26556299, 28199314 \\
\hline TSR2 & 28600339 \\
\hline TUBB4A & OMIM Myeloid Leukemia \\
\hline TWIST1 & OMIM Myeloid Leukemia \\
\hline TYR & OMIM Cancer \\
\hline TYW1 & 25426837 \\
\hline U2AF1 & 27276561,25426837 \\
\hline U2AF2 & 27276561,25426837 \\
\hline UBE2T & 28600339 \\
\hline UBR5 & 25426837 \\
\hline UFSP2 & OMIM Myeloid Leukemia \\
\hline$U R O D$ & $24429628,29489754,29625052$ \\
\hline VHL & OMIM Cancer, 24429628, 29489754, 29625052, 26556299, 28199314 \\
\hline VHL1 & 26580448 \\
\hline VPS11 & OMIM Myeloid Leukemia \\
\hline WAS & $24429628,29489754,29625052,28199314$ \\
\hline WHSC1L1 & OMIM Myeloid Leukemia \\
\hline
\end{tabular}




\begin{tabular}{|l|l|}
\hline WRAP53 & 28600339 \\
\hline WRN & $24429628,29489754,29625052,28199314$ \\
\hline WT1 & $\begin{array}{l}\text { OMIM Cancer, 24429628, 27276561, 29489754, 29625052, 25426837, } \\
26556299,2658044\end{array}$ \\
\hline WWOX & OMIM Cancer \\
\hline XBP1 & 25426837 \\
\hline XIAP & 26556299 \\
\hline XPA & $24429628,29489754,29625052,28199314$ \\
\hline XPC & $24429628,29489754,29625052,28199314$ \\
\hline XPO1 & 25426837 \\
\hline XRCC2 & 28600339,28199314 \\
\hline XRCC3 & OMIM Cancer, 29489754 \\
\hline YAP1 & 26556299 \\
\hline ZBTB16 & OMIM Myeloid Leukemia \\
\hline ZEB2 & 27276561 \\
\hline ZFHX3 & OMIM Cancer \\
\hline ZNF471 & 25426837 \\
\hline ZRSR2 & 27276561,25426837 \\
\hline
\end{tabular}

After these filters were applied, we were left with 5,880 rare variants $(3,934$ unique) in the cohort, among which the vast majority (93.4\%) were missense, with $2.6 \%$ in-frame indels, $2.6 \%$ truncating, and $0.9 \%$ splice site variants (Figure $5)$.

Figure 4: Overview of patient and variant characteristics. 

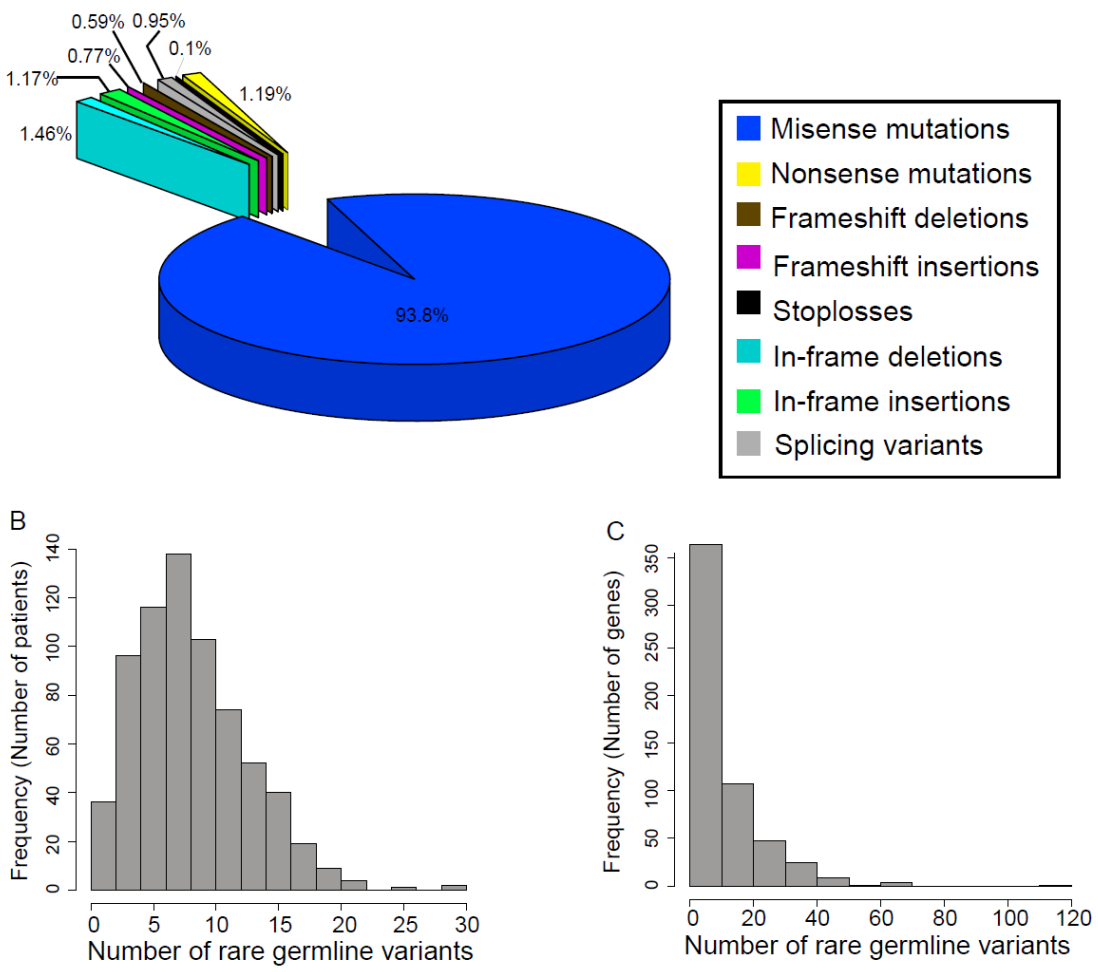

Figure 4: (A) Distribution of rare variant effects on proteins. (B) Distribution of rare germline variant burden by patient. (C) Distribution of rare germline variant burden by gene.

\section{Chapter 2-4: Filtering putative compound heterozygotes and rare}

\section{homozygotes}

For compound heterozygotes, we first extracted all variant pairs that appeared in the same gene and patient. We next computed the linkage disequilibrium measure $r$ for each pair, using LDlink (Machiela and Chanock 2018), removing any pair with $r>0$ and $r^{2}>0.1$ as likely being in cis. Finally, we manually examined reads using IGV (Robinson, Thorvaldsdottir et al. 2011). Any pairs of variants appearing on the same fragment were removed as being in cis. Since standard variant-calling algorithms often have difficulty accurately determining 
the alleles in long insertions and deletions polymorphisms, we omitted long ( $>9$ bp) indels from consideration when querying for rare allele homozygotes and compound heterozygotes.

\section{Chapter 2-5: Statistical testing}

All statistical testing was performed using the $\mathrm{R}$ software. To determine the null frequency of compound heterozygotes and rare allele homozygotes in a gene with $n$ rare variants across the cohort, we implemented a permutation scheme. For each permutation, we first assigned rare variant-carrying status to $n$ of the 2 $x 690=1380$ chromosomes. Chromosomes were then randomly paired, and the number of pairs where both chromosomes were rare variant-carrying was recorded (these pairs represent the compound heterozygote/rare allele homozygote patients for that permutation). To generate null values for a collection of 21 Fanconi anemia genes, the permutation was performed for the observed numbers of rare variants $\left(n_{1}, n_{2}, \ldots, n_{21}\right)$ in each of these genes and the result was summed. The null distribution was generated from 100,000 such permutations. The $P$-value was assigned by comparing observed value to this distribution, which also yielded the confidence interval.

All differences in proportions were assessed using a test of equal proportions (the function prop.test in R). Differences in rare-variant burdens between different classes of genes were tested using a Wilcoxon test. Difference in overall survival was tested using the Cox proportional hazards model, adjusting for patient age. Concordance between MPO mutational status and cytogenetic lesions, and 
between mutation status in pairs of genes, was tested using Fisher's exact test with the R package exact2x2.

\section{Chapter 3: Results}

Chapter 3-1: Rare variants in genes with known roles in germline myeloid malignancy susceptibility

Introduction

The World Health Organization (WHO), the Society for Hematopathology and the European Association for Haematopathology, published the third edition of the WHO Classification of Tumours of Haematopoietic and Lymphoid Tissues in 2001 (Swerdlow, 2008). Since then, the classification has been constantly updated as researchers and clinicians gain more information about myeloid malignancies. Although most cases of myeloid malignancies are sporadic diseases, there is a subgroup of cases which clearly have germline predisposition corresponding to certain genes which cause the diseases (Baptista, 2017). With the advancement in sequencing technologies, researchers and clinicians can now incorporate genetic changes in diagnosing myeloid malignancies. As a result, the World Health Organization (WHO) revised their classification of myeloid neoplasms and acute leukemia to include a new category, termed "myeloid neoplasms with germline predisposition" in 2016 (Arber, Orazi et al. 2016). The category is summarized in Table 9.

Table 8: Classification of myeloid neoplasms with germ line predisposition 


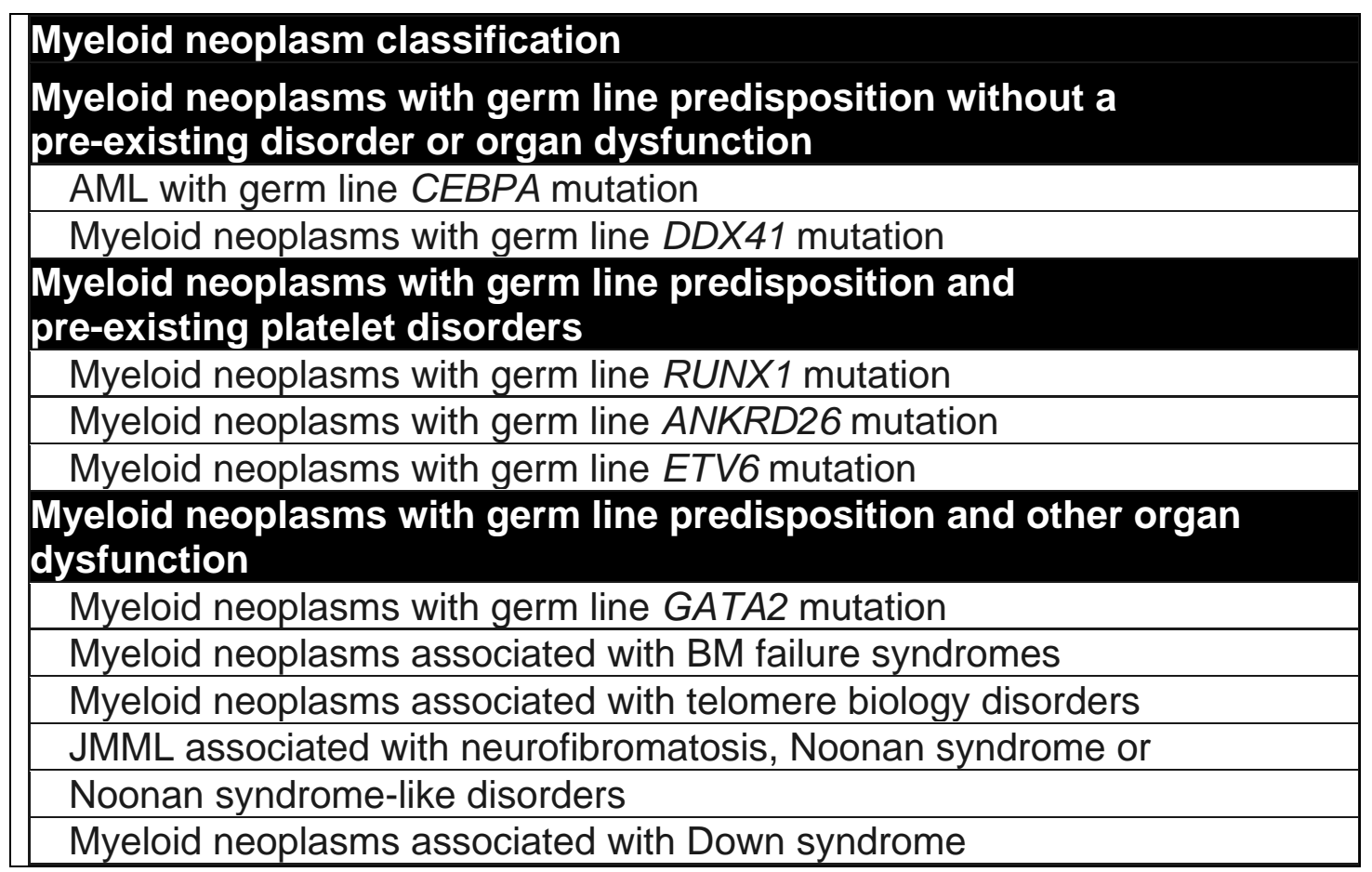

Result

We identified 11 patients in our cohort who would fit the criteria owing to mutations in DDX41 (Figure 5), four of whom harbored previously-described (Polprasert, Schulze et al. 2015, Cheah, Hahn et al. 2017, Guidugli, Johnson et al. 2017, Makishima, Yoshizato et al. 2018) DDX41 variants with strong evidence for disease roles. One of the four patients also harbored a somatic DDX41 mutation (p.R525H) in trans with his germline variant (p.I396T) (Polprasert, Schulze et al. 2015).

Also specified in the $2016 \mathrm{WHO}$ criteria are germline mutations in ANKRD26, CEBPA, ETV6, GATA2, and RUNX1. Although 31 patients had rare ANKRD26 variants, none had variants in the $5^{\prime}$ untranslated region, which has been the site of nearly all causal $A N K R D 26$ variants identified to date (Noris, Perrotta et al. 
2011). No CEBPA mutations were observed, but five patients were found to have ETV6 mutations (Figure 5). One such patient carried a p.R353Q mutation affecting the highly-conserved ETS domain, a well-known hotspot for both germline and somatic mutations in ETV6 among myeloid malignancy patients (Zhang, Churpek et al. 2015). The p.R353Q variant is exceedingly rare, present at allele frequency $2.83 \times 10^{-5}$ in the gnomAD database (Karczewski, 2019), and has a hybrid score of 0.83 . Three patients with GATA2 mutations and six with RUNX1 mutations were found (Figure 5). The RUNX1 variants in four of the six patients were novel (neither present in any of the population databases nor assigned rsIDs), and three were unanimously deemed deleterious by the six scoring algorithms, including two alleles (p.R166Q and p.Y287X) that were previously found (Song, Sullivan et al. 1999, Michaud, Wu et al. 2002) through pedigree analyses of families with familial platelet disorder with predisposition to acute myeloid leukemia (FPD/AML). In our cohort, both individuals carrying these latter alleles were pediatric cases, with ages-of-onset 1 and 12 years.

Figure 5: Germline mutations in genes implicated in myeloid neoplasms with germline predisposition.

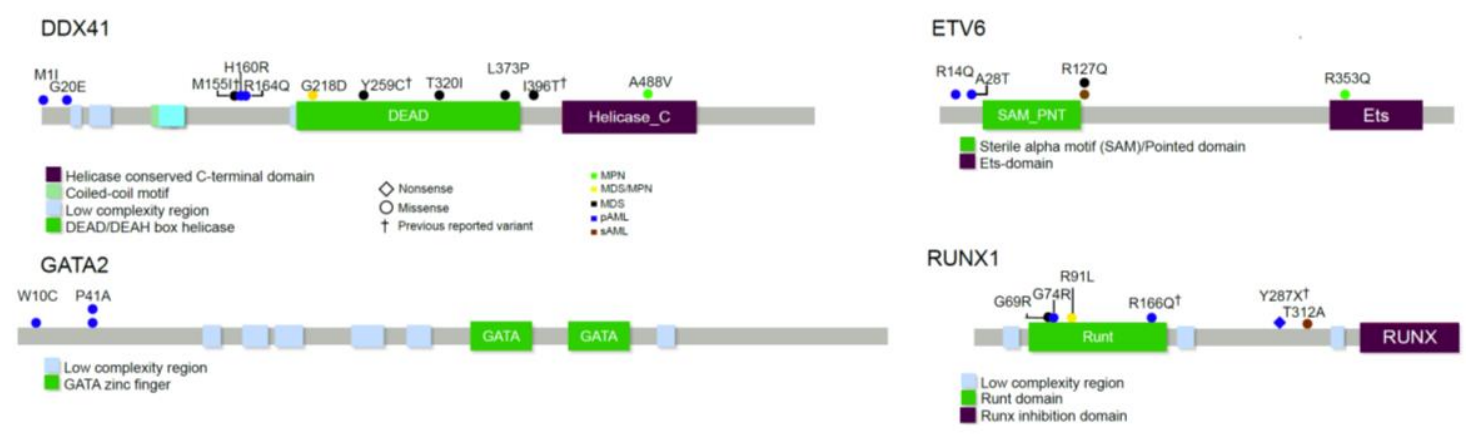


Figure 5: Color indicates patient diagnosis and shape indicates impact on amino acid sequence. Specific variants previously reported as susceptibility alleles are indicated with a dagger $(\dagger)$.

Other genes associated with familial MDS are SAMD9/SAMD9L, SRP72, MECOM, and TERT. SAMD9/SAMD9L are adjacent homologs on chromosome $7 q 21.2$ that have recently been implicated by several groups in a substantial portion of pediatric bone marrow failure patients with monosomy 7 (Schwartz, Ma et al. 2017, Bluteau, Sebert et al. 2018). We identified 42 patients with rare SAMD9/SAMD9L variants, though none are pediatric cases with monosomy 7 . No clearly deleterious variants were found in the other genes. Overall, therefore, our cohort included 25 patients (3.6\%) with variants that would qualify them as having germline predisposition, including six $(0.9 \%)$ harboring previously identified, likely causal variants.

\section{Discussion}

Here we show that our cohort has 25 patients $(3.6 \%)$ that would qualify as having germline predisposition, including 6 harboring previously identified, likely causal variants. Those variants are found in DDX41, ETV6, GATA2 and RUNX1. Recent studies have shown that there are numerous pathogenic or risk factor variants on those genes associated with myeloid malignancies. (Polprasert, 2015) (Walker, 2002) (Lewinsohn, 2016) (Li, 2016) (Cardoso, 2016) (Zhang, 2015) (Landrum, 2018) (Hahn, 2011) (Luo, 2019). The fact that our study can capture these germline variants strengthens the fact that myeloid malignancy has heritable components. However, since most of those variants were discovered in familial settings, there is a possibility that there are variants which remain 
undiscovered in the population. DDX41, ETV6, GATA2 and RUNX1 are well known to be mutated somatically in myeloid malignancy (Polprasert, 2015) (Fisher, 2017) (Harada, 2004) (Wang, 2014) (Barjesteh van Waalwijk van DoornKhosrovani, 2005) (Sood, 2017) At the same time, patients who have these germline variants are known to have an increased risk of developing myeloid neoplasms, including MDS, AML, and chronic myeloid leukemia later in their lives (Lewinsohn, 2016) (Gao, 2019). These observations support the idea that the somatic aspects of human malignancies can offer clues to guide the search for inherited predisposition variants. Genes that are important for malignancy onset and progression tend to be those that acquire mutations somatically, or that are subject to somatic duplication or deletion events. Therefore, these same genes make attractive candidates in the search for important germline mutations. Understanding the interaction between germline variants and somatic mutation in myeloid malignancies will give a better picture of the gene and pathway disruptions that lead to disease phenotypes, eventually yielding new therapeutics.

\section{Chapter 3-2: Pathogenic rare variant burden and MPO as a candidate}

\section{predisposition gene}

\section{Introduction}

Next generation sequencing is becoming more popular in both research and clinical diagnosis. As a result, researchers are able to discover new variants. This creates challenges in determining whether the newly found variants are 
associated with the disease and how the variants affect disease phenotypes. In order to solve these problems, there is a need to have a standard guideline in variant classification for both researchers and clinicians. Therefore, a workgroup consisting of American College of Medical Genetics (ACMG), Association for Molecular Pathology (AMP), and College of American Pathologists, representing clinical laboratory directors and clinicians, was formed in 2013 to create a recommendation for the use of standard terminology for classifying sequence variants. Using the recommendation, variants that are found can be classified based on various evidence weighted according to an established system (Richards, 2015).

The ACMG standards and guidelines for the interpretation of sequence variants is a five-tier terminology system using the terms "pathogenic," "likely pathogenic," "uncertain significance, "likely benign," and "benign". Pathogenic variants refer to variants which are proven to be disease-causing whereas likely pathogenic variants are variants which are very possible, more than $90 \%$ of chance, to be disease causing. We first examine both pathogenic and likely pathogenic variants because these variants can have large effects on both protein production and formation. Our analysis of these variants show MPO as a candidate predisposition gene.

\section{Result}

A total of 146 variants were classified as pathogenic or likely pathogenic (Figure $6 A)$, spread across 65 genes and 137 patients (19.9\%). The gene with the 
largest pathogenic/likely pathogenic variant burden is $M P O$, which harbors such a variant in 19 of the patients. MPO (myeloperoxidase) codes for a heme protein produced during myeloid differentiation. Neutrophil azurophilic granules have MPO as their major component, and MPO is expressed mostly in blood cells. The pathogenic variants found in our cohort are dominated by two substitutions: a splice site variant (c.2031-2A>C; eleven patients), and a missense variant (c.C1705T, p.R569W; seven patients) (Figure 6B). Multi-species alignment shows that both of the corresponding wild-type alleles are highly conserved. An earlier report associated the c.2031-2A>C variant with myeloperoxidase deficiency, and transfection of a construct harboring the variant resulted in mRNA with 109 nucleotides inserted between exons 11 and 12, yielding a protein that should lack enzymatic activity (Marchetti, Patriarca et al. 2004). The variant was also identified as a being associated with protein abundance, both in cis (MPO) and in trans (RAB26), in human plasma (Sun, Maranville et al. 2018).

To examine the prevalence of these pathogenic MPO alleles in control and other case cohorts, we queried the gnomAD database (Karczewski, 2019) of 60,144 control exomes, as well as $531 \mathrm{AML}$ patient exomes from the recently-published Beat AML study (Tyner, Tognon et al. 2018). Frequencies of both alleles are significantly higher in our cohort $(0.8 \%$ and $0.51 \%$ for $\mathrm{c} .2031-2 \mathrm{~A}>\mathrm{C}$ and p.R569W, respectively) than in the gnomAD database $(0.38 \%$ and $0.13 \% ; \mathrm{P}=$ 0.012 and $4.0 \times 10^{-4}$, respectively). The allele frequencies in the Beat AML study $(0.8 \%$ and $0.6 \%)$ are similar to those in our cohort. As in gnomAD, both variants 
have similarly lower frequencies in the Haplotype Reference Consortium (HRC) (McCarthy, 2016) population database (Figure 6C).

We also examine the correlation between MPO variants and patients' cytogenetic lesions. In our cohort, patients with rare variants in MPO are more likely to have cytogenetic lesions, particularly complex karyotype and deletion of chromosome 5 (Figure 6D), as are those with pathogenic MPO variants in particular (Figure $6 \mathrm{E})$.

Figure 6: Myeloperoxidase (MPO) as a candidate susceptibility gene in myeloid malignancy.

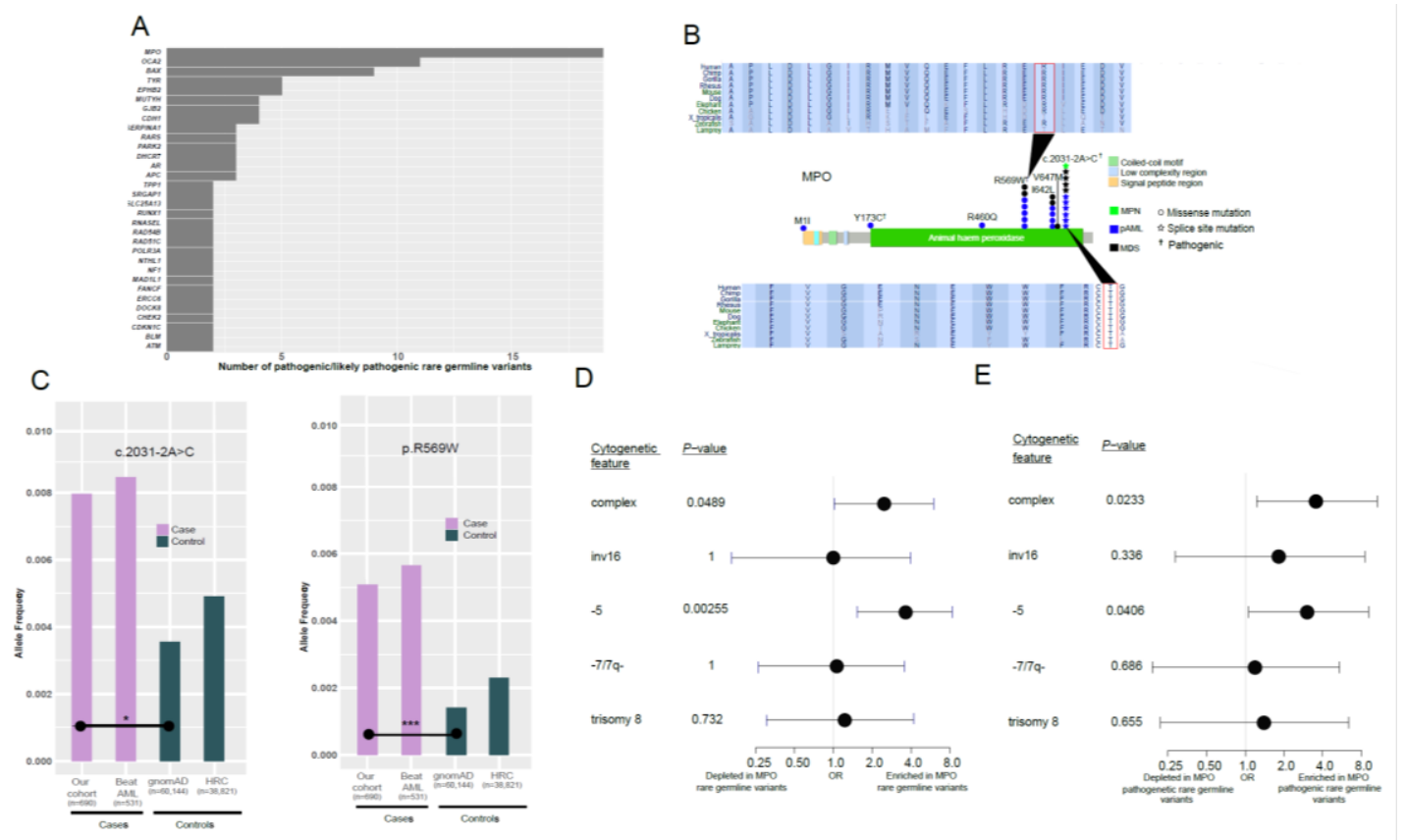

Figure 6: (A) Genes ranked by pathogenic/likely pathogenic variant burden. For clarity, only genes with more than one such variant are shown. (B) Rare protein-altering variants in MPO. Pathogenic/likely pathogenic variants are indicated with a dagger $(\dagger)$. Patient diagnosis is indicated by color and effect on amino acid sequence is indicated by shape. Also shown is multi-species alignment in regions surrounding the amino acid residue altered by the missense variant $p$. R569W (top) and the nucleotide residue altered by the splice variant c.2031-2A $>C$ (bottom). (C) Allele frequencies of case and control cohorts for the two most recurrent pathogenic alleles in MPO. For 
clarity, significance is only shown for comparisons between our cohort and gnomAD. ${ }^{*} P<0.05$; ${ }^{* * \star} P<0.0005$. (D, E) Associations between the presence of cytogenetic lesions and the presence of a rare MPO variant (left) or pathogenic rare $M P O$ variant (right). $\mathrm{OR}=$ odds ratio. Filled circles indicate OR estimate, with whiskers indicating a 95\% confidence interval for the OR.

\section{Discussion}

Here, we show that MPO has the highest pathogenic rare germline variant burden. In fact, $M P O$ is an appealing target for myeloid malignancies as there is growing evidence showing that there are relationships among MPO, inflammation, and cancer (Hanahan, 2011) (Al-Salihi, 2015) (Grivennikov, 2010). In fact, MPO polymorphism is linked to abnormal MPO expression and greater risks of various diseases (Ding, 2013) (Zhang, 2001). A previous study showed that the p.R569W variant, one of the driving signals, can cause defects in MPO (Nauseef, 1994). It is also known that MPO can produce oxidants which can lead to DNA damage, and thus promote mutagenesis (VanderVeen, 2001).

Myeloperoxidase is a hallmark enzyme of acute myeloid lineage. Khan et al. reported that in AML patients, there are higher plasma MPO levels (range 1.0$9514 \mathrm{ng} / \mathrm{mL}$ ) as compared to healthy subjects (range 3.5-20.6 ng/mL) (Khan, 2018). Furthermore, patients with high endogenous levels of MPO are found to have higher chance of developing cancer (He, 2009).. 
Chapter 3-3: Exploring the overall germline rare variant burden and investigating two-hit hypothesis among germline rare variants and with somatic mutations.

\section{Introduction}

In 1971 Knudson proposed that retinoblastoma was initiated by inactivation of a putative tumor suppressor, the Rb1 gene (Knudson, 1971). According to a "twohit" model, dominantly inherited predisposition to cancer starts with a germline mutation. In order to initiate tumorigenesis, a second hit is required. The second hit often comes as somatic mutation which patients acquire later in their lives. On the other hand, non-hereditary cancer also requires the same two hits, but both are somatic.

Knudson's two-hit hypothesis can also apply to solely germline variants when those variants are either homozygous or compound heterozygous. Homozygosity is a situation in which there are two identical alleles at one specific site, one inherited from each parent.

On the other hand, compound heterozygosity occurs when two mutations affect one gene in two different positions. The two mutations may exist either in the same copy (in cis heterozygosity) or in both paternal and maternal alleles, each at a different position (in trans compound heterozygosity). In the in trans case, both alleles may behave abnormally and cause a disease.

In order to assess whether the variant pair are an in cis or in trans arrangement, one can look at the linkage disequilibrium (LD) between the variants. LD refers 
to the non-random association of alleles at linked loci. Variants which are in linkage disequilibrium are considered to be correlated with each other and this also indicates that the variant pair is in cis arrangement. The degree of linkage disequilibrium can be measured by D', an indicator of allelic segregation for two genetic variants, and $\mathrm{R}^{2}$, a measure of correlation of alleles for two genetic variants (Machiela, 2018).

\section{Result}

Examination of rare variant burden across all 657 genes under consideration (104 of which have no rare variants in our cohort; Figure 7A), irrespective of pathogenicity, shows that genes associated with Fanconi anemia (FA) and, in general, those associated with autosomal recessive (AR) disorders, have higher rare variant burdens than do the other genes under consideration (Wilcoxon test $P=0.002$ and 0.027 , respectively; Figure 7B). We also observe an enrichment of truncating variants (nonsense, frameshift, and splice site) in FA and AR genes (Figure 7C,D). The 205 truncating variants are spread across 167 patients and 119 genes. Of these variants, 97 (47.3\%) were found in AR genes and 18 (8.9\%) were found in FA genes, proportions higher than the proportions of all variants $(29.0 \%$ in AR genes, $P=1.3 \times 10^{-8}$ and $6.6 \%$ in FA genes, $P=0.14$ ) and proportions of all genes under consideration $\left(25.0 \%\right.$ AR genes, $P=1.0 \times 10^{-9}$ and $3.2 \%$ FA genes, $\left.7.7 \times 10^{-4}\right)$. Moreover, the proportion of rare variants that are truncating is significantly higher in AR genes (5.7\%) and FA genes (4.6\%) than in other genes $\left(2.5 \% ; P=1.5 \times 10^{-9}\right.$ and 0.013 , respectively). 
Figure 7: Autosomal recessive genes and Fanconi anemia genes have higher overall and truncating rare germline variant burden. 

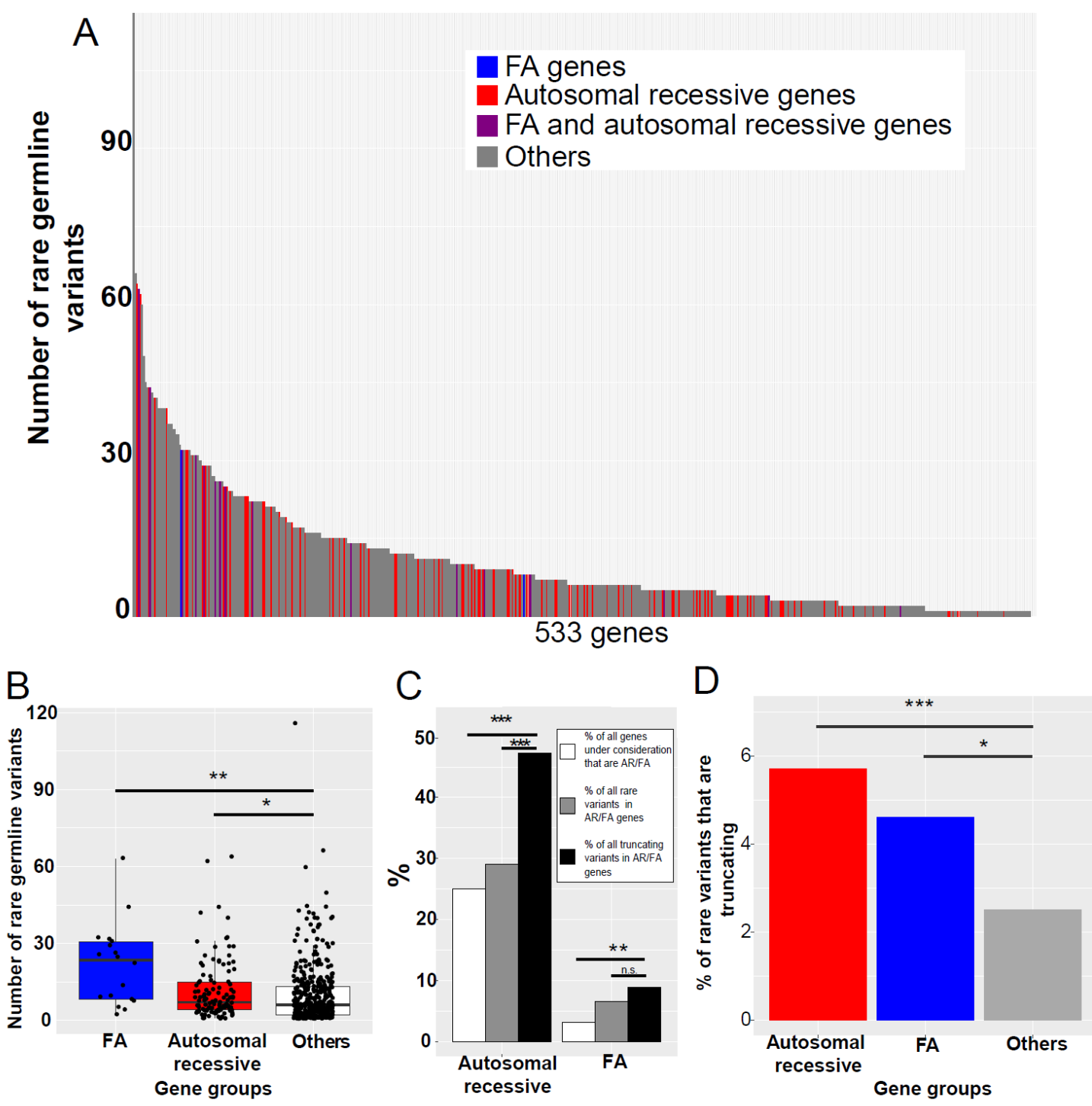

Figure 7: (A) Each gene (with at least one germline variant) is represented by a small bar, whose height indicates the number of rare germline variants observed in that gene. A bar is colored if its corresponding gene is autosomal recessive (red), a FA gene (blue), or both (purple). (B) Rare germline variant burden for genes, classified by Fanconi anemia and autosomal recessive status. ${ }^{*} P<0.05 ;{ }^{* *} P<0.005$. (C) Bars indicate (from left to right) proportions of all truncating rare germline variants that are in AR/FA genes, proportions of all variants that are in FA/AR genes, and proportion of all genes under consideration that are FA/AR genes. ${ }^{* *} P<0.001 ;{ }^{* *} P<10^{-7} ;$ N.S.: not significant at 0.05 level. 
An AR gene harboring a rare disruptive variant may have the other parental copy disrupted through several possible mechanisms, one of which is inheritance of a rare variant on the other parental allele. This biallelic scenario may either take the form of a rare allele homozygote $(\mathrm{RAH})$, or of a compound heterozygote $(\mathrm{CH})$. Unsurprisingly, RAHs were exceedingly rare in our data set, with only 17 present. Interestingly, the only gene harboring a RAH in more than one patient was BRCA2 (also known as FANCD1). Among the three patients carrying BRCA2 RAHs was one diagnosed at 15 years, and a 57-year-old who died eight months after diagnosis (the third patient did not have age or survival information available). In general, patients with rare germline $B R C A 2$ variants, whether in a heterozygous or homozygous constellation, have significantly worse outcomes with regard to overall survival (Figure 8B; age-adjusted hazard ratio 2.62, 95\% Cl 1.26-5.44, $P=0.0072)$.

Patients with $\mathrm{CHs}$ were also identified. Compound heterozygosity here is sometimes putative, because in silico it is only possible to conclusively determine whether the two variants are in trans (thereby affecting both parental homologs) or in cis (affecting one homolog at two loci) if read pairs cover both loci (see Patients and Methods). We observed a total of 95 putative CHs, across 85 different patients and 71 unique genes. Among the $95 \mathrm{CHs}, 30$ were in AR genes (Figure $8 \mathrm{C}$ ), with two in BRCA2 (Figure 8D). A larger proportion of $\mathrm{CH}$ and $\mathrm{RAH}$ are in Fanconi anemia genes, compared to the proportion of all rare variants in FA genes $(10.7 \%$ vs. $6.6 \% ; P=0.06)$ and compared to the proportion of FA 
genes among all genes under consideration $\left(10.7 \%\right.$ vs. $\left.3.2 \% ; P=6.4 \times 10^{-5}\right)$, though the former does not attain statistical significance.

Under the null hypothesis of random assortment of affected chromosomes, we could calculate the number of $\mathrm{RAH} / \mathrm{CH}$ that would be expected in a gene given the total number of rare variants present in that gene across the cohort. A higherthan-expected number of $\mathrm{RAH} / \mathrm{CH}$ events is indicative of a disease predisposition for individuals with biparental inheritance of rare variants in the gene. Using this principle, we tested for enrichment of $\mathrm{RAH} / \mathrm{CH}$ across all FA genes (Figure 8E) given the total number of rare alleles present in these genes across patient chromosomes (see Patients and Methods). Assuming random assortment, we would expect 4.6 (95\% confidence interval 1 - 9) patients to have $\mathrm{RAH} / \mathrm{CH}$ in FA genes, but we observe $12(P=0.002)$.

Figure 8: Genes with rare variants in both copies in the same patient. 
A

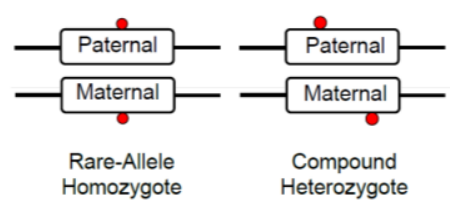

C$$
\text { C }
$$

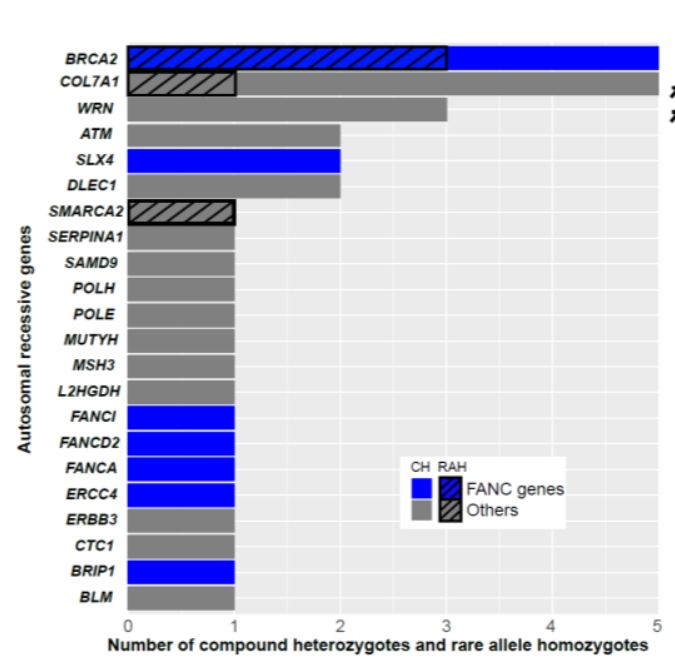

B

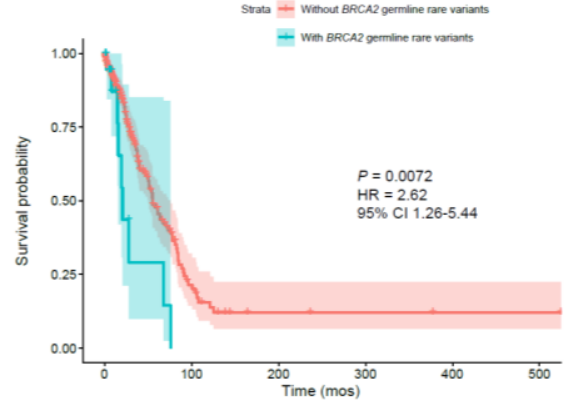

BRCA2

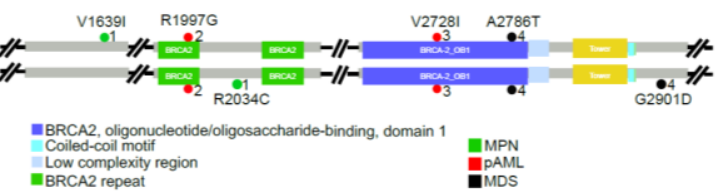

$\mathrm{E}$

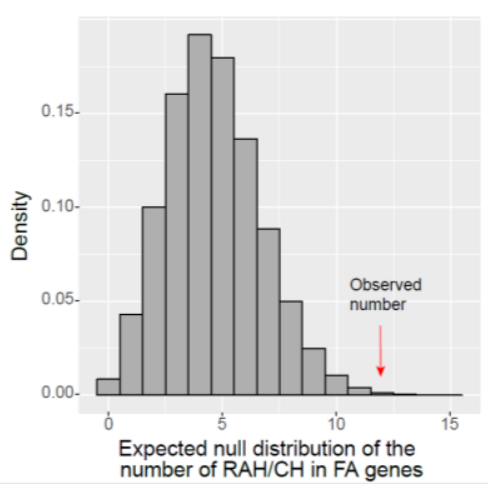

Figure 8: (A) Both parental homologs may be impacted by rare germline variants either though homozygosity (RAH; left) or compound heterozygosity ( $\mathrm{CH}$; right). (B) Presence of rare germline $B R C A 2$ variants are associated with poor overall survival. (C) Counts for instances of $\mathrm{RAH} / \mathrm{CH}$ in autosomal recessive genes. These categories combined are enriched for Fanconi anemia genes. (D) $B R C A 2$ has five such $\mathrm{RAH} / \mathrm{CH}$ pairings in four different patients. Individual patients are indicated by distinct numbers 1-4 adjacent to their variants. Patient diagnosis is indicated by color. 
Figure 9: Global and patient-specific concordance of rare germline variants and somatic mutations in each gene. 

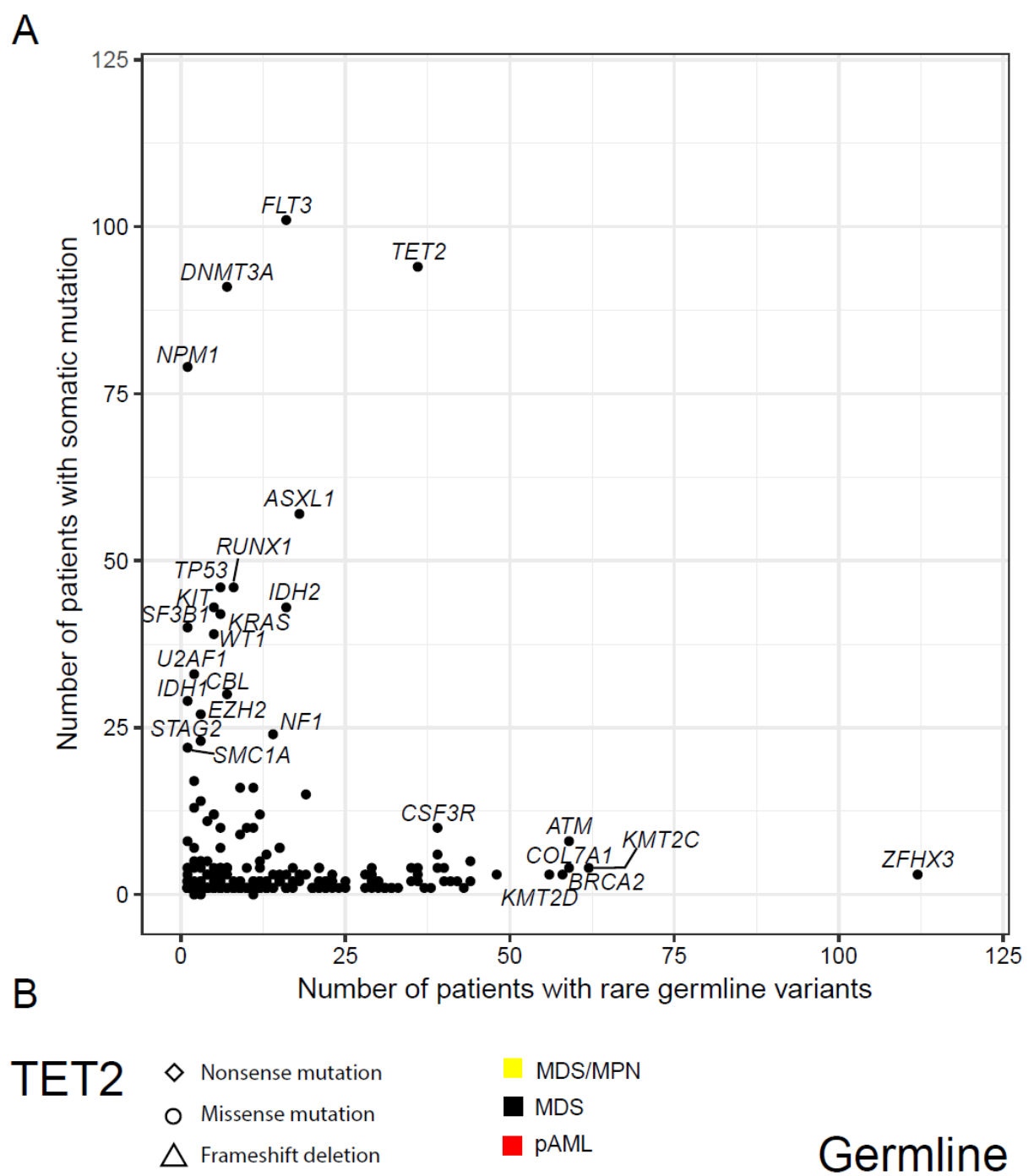

\section{Germline}

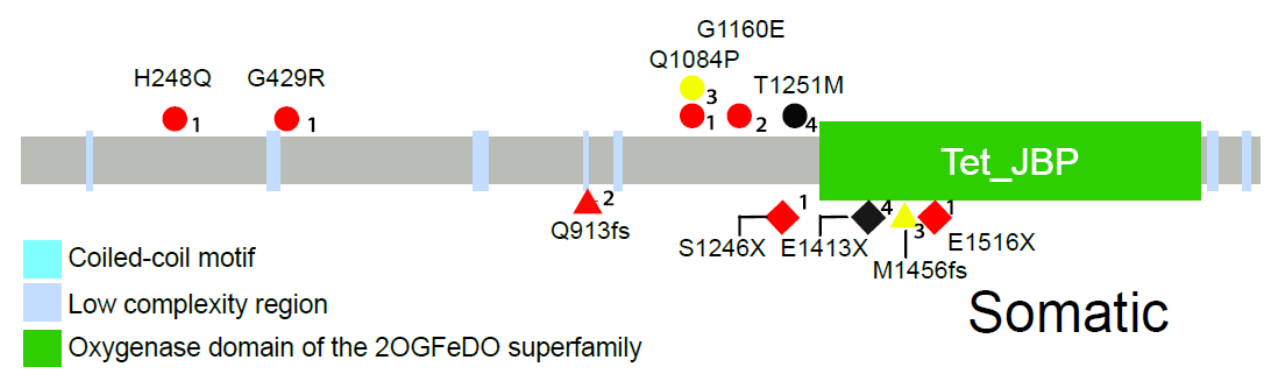

Figure 9: (A) The genes with high overall germline variant burden (horizontal axis) do not tend to be those with high overall somatic mutation frequency (vertical axis). TET2 is one of the few genes with high overall frequencies of both somatic and rare germline variants. (B) Rare germline variant-somatic mutation pairs in the same patient are shown. Individual patients are indicated by distinct numbers 1-4 adjacent to their variants. Patient diagnosis is indicated by color and effect on amino acid sequence is indicated by shape. 


\section{Discussion}

The FA pathway is known to be an essential DNA repair pathway, and deficiency of FA genes result in Fanconi anemia, and hence increase the chance of having myeloid malignancies. In line with our finding, Maung et al. also show that there is an enrichment of deleterious germline rare variants on FA genes in $A M L$ patients (Maung, 2018).

Previous studies have demonstrated that patients with FA will have an earlier onset of childhood cancers if they carry biallelic BRCA2 mutations (Offit, 2003) (Howlett, 2002) (Hirsch, 2004). It is also known that biallelic BRCA2 mutations would increase the risk of AML, acute T-cell lymphoma, medulloblastoma and Wilms tumor (Wagner, 2004) (Hirsch, 2004) (Loizidou, 2016), and it is associated with shortened lifespan (Alter, 2007) (Wang, 2007). 


\section{Chapter 4: Discussion \& Future Directions}

The first population-based germline rare variants study of myeloid malignancy Here we have presented results from the analysis of the largest collection, to our knowledge, of myeloid malignancy germline exomes reported to date. Most publications of large myeloid neoplasm patient exome cohorts have focused on the characterization of somatic mutations (Cancer Genome Atlas Research 2013) (Makishima, Yoshizato et al. 2017) (Tyner, Tognon et al. 2018). The dearth of germline genome-wide analysis studies published to date in these diseases may partially be the result of publication bias, as most efforts have likely focused on common variants represented on genotyping arrays, and probably yielded negative results. One exception is a recent GWAS in myeloproliferative neoplasms (Tapper, Jones et al. 2015), which identified common risk variants from a large meta-analysis of array data.

In our cohort of 690 patients, we were able to identify previously-reported, likelycasual variants in approximately $1 \%$ of patients. Overall, we identified $25(3.6 \%)$ patients with a disease that could be classified as myeloid neoplasms with germline predisposition, according to the 2016 WHO category. We lacked family history information for most of these patients, so we could not determine whether there was evidence for familial disease tracking with these variants. There were no statistical differences in age-of-onset between these 25 patients and the rest of the cohort, although there were three under 13 years of age. 


\section{Whole genome sequencing instead of whole exome sequencing}

Technology advancement enables researchers to explore the contribution of lowfrequency and rare genetic variants in disease phenotypes, for example this project. By focusing the protein coding region in the human genome, which is about $2 \%$ of the whole human, researchers are already able to identify new genes implicated in various human diseases. Since the cost of next generation sequencing is lower, attention has turned to exploring the remaining $98 \%$ of the human genome. Here we show how germline rare exonic variants contributes to myeloid malignancies. However, the contribution from germline rare non-coding variants is still an unknown. Understanding the contributions from those variants can explain the rest of the missing heritability of myeloid malignancies, and hence discover novel targets for treatments.

However, there are still challenges ahead in understanding rare variants, one of which is that variant frequency can be different from one population to another population as there are isolates or different ethnic groups. To deal with this challenge, studies on different populations are necessary in order to determine truly rare variants. Also, although whole genome sequencing in low-depth coverage is available, whole genome sequencing with high-depth coverage will be beneficial in understanding the contribution of germline rare non-coding variant. The third problem is to validate the in silico finding using either in-vitro or in-vivo experiments in order to know impacts of these rare variants. Despite 
these challenges, studying the contributions of germline rare variants in the human genome will be worthwhile (Zuk et al. 2014).

\section{MPO as a novel myeloid malignancy predisposition gene}

Our analysis revealed a novel candidate risk gene, myeloperoxidase (MPO), with two pathogenic variants in particular showing elevated frequencies both in our cohort and in a recently-published independent cohort of AML patients (Tyner, Tognon et al. 2018). MPO is an appealing candidate as a myeloid malignancy susceptibility gene for a number of reasons. It is expressed, on both the mRNA and protein levels, most prominently in blood cells (Consortium 2015) (Schmidt, Samaras et al. 2018). High MPO expression is characteristic of acute promyelocytic leukemia (APL), though none of the carriers of the MPO rare variants here have the $t(15 ; 17)$ translocation causing APL. Germline variants in MPO (including one of those with elevated frequency in our cohort) have been associated with high counts of leukocytes, monocytes, neutrophils, and other blood cell types (Astle, Elding et al. 2016).

\section{The importance of FA genes in myeloid malignancies}

Fanconi anemia (FA) genes were a category that emerged recurrently in our analysis. We found that, compared to other genes under consideration, FA genes were enriched for overall rare germline variant burden, truncating mutations, and compound heterozygotes/rare allele homozygotes. Even after accounting for the total FA rare germline variant content in patient chromosomes, the number of patients for which affected FA genes are paired is significantly higher than would 
be expected by chance. Given that nearly all FA genes show an autosomal recessive pattern in hematologic malignancies, this implies that observed FA enrichment is not random, and suggest biparental inheritsance of disruptive variants in an FA gene as a myeloid malignancy risk factor. Of all genes under consideration, the FA gene BRCA2 had the highest incidence of compound heterozygosity/rare allele homozygosity in the patient cohort. In general, patients with rare $B R C A 2$ germline variants had worse outcomes. Although $B R C A 2$ is associated with autosomal recessive inheritance in $\mathrm{FA}$, it is considered an autosomal dominant gene in cancer. In the current setting, therefore, germline variants here may be acting in a dominant manner in myeloid malignancy severity, or the other BRCA2 copy may be compromised via other mechanisms. The latter may also be true for the abundance of heterozygous truncating mutations observed in autosomal recessive genes in general.

\section{Crosstalk between germline rare variants and somatic mutations in the same}

\section{patient}

We queried the data for synergy between germline and somatic contributors to disease, but were unable to detect anything statistically significant. We observed no evidence of germline and somatic variants cooperating, with no pairs (either within the same gene or in separate genes) occurring more than would be expected by chance. However, querying all possible pairs entails an enormous number of tests, and we were likely severely under-powered for any such test to withstand the necessary multiple-hypothesis corrections. 


\section{Improvement in sample size}

This study can be further expanded by analyzing more patient sequencing data.

Our data set comprised in total 690 myeloid malignancy patient whole exomes. It is suggested that a discovery sample of at least 25,000 subjects and a substantial replication set is needed for a well-powered study that aims to identify rare variants (Zuk et al. 2014). However, over time, there will be more myeloid malignancy patients sequencing available throughout different databases such as dbGaP, The European Genome-phenome Archive (EGA), St. Judes Cloud PeCan, Genomic Data Commons Data Portal, etc. All these databases hold large sequencing data sets that researchers can use to discover rare variant contributions to various human diseases. Also, collaboration with different research teams and forming large consortiums can effectively solve the sample size problem.

Table 9: Number of whole-genome and whole-exome patient samples available from public sources

\begin{tabular}{|c|c|c|c|c|c|}
\hline \multirow{3}{*}{$\frac{\text { Resourc }}{\underline{\mathrm{e}}}$} & \multirow{3}{*}{ Website URL } & \multicolumn{2}{|c|}{$\frac{\text { Acute myeloid }}{\text { leukemia }}$} & \multicolumn{2}{|c|}{$\frac{\text { Myelodysplastic }}{\text { syndrome }}$} \\
\hline & & WGS & Wxe & Wea & wya \\
\hline & & whes & Was & whes & Was \\
\hline dbGaP & 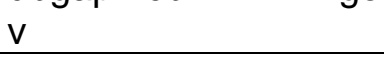 & 1336 & 178 & 0 & 16 \\
\hline EGA & ebi.ac.uk/ega/home & 157 & 78 & 5 & 529 \\
\hline GDC & gdc.cancer.gov & 229 & 138 & 0 & 0 \\
\hline ICGC & icgc.org & 32 & 254 & 0 & 0 \\
\hline Pecan & pecan.stjude.cloud & 106 & 360 & 0 & 4 \\
\hline
\end{tabular}




\section{Limitations of our study}

Our study has a number of limitations. First, our use of control data was limited, since the patient-level variant data is not provided on public websites such as gnomAD's. As such, it is not possible to determine, for example, the number of patients harboring variants in a particular collection of genes, or to identify compound heterozygotes. Second, although the collection of myeloid malignancy exomes analyzed here is the largest to date, we are substantially underpowered to detect case/control differences in rare-allele burden, even after restricting to our list of 657 genes. It follows that validation studies are needed for many of the results presented here. Third, determining whether pairs of variants in the same gene and patient were in cis or in trans was sometimes not possible. Nonetheless, our permutation approach (see Patients and Methods) to testing for elevated levels of homozygosity/compound heterozygosity in a gene provides a framework for performing such tests using whole exome cohorts in general.

\section{Conclusion}

Overall, this study sheds light on some candidate mechanisms by which inherited alleles may confer risk of myeloid malignancy. The identification of such alleles has several practical benefits. Individuals carrying susceptibility alleles may benefit from increased surveillance, facilitating early preventative treatment. These individuals may also benefit from genetic counseling. Asymptomatic family members who are carriers of the deleterious allele may be excluded as candidate bone marrow donors. Finally, understanding the mechanisms-of-action of these 
alleles will help elucidate disease biology, potentially leading to improved patient treatment. 


\section{Bibliography}

Adzhubei, I. A., Schmidt, S., Peshkin, L., Ramensky, V. E., Gerasimova, A., Bork, P., ... Sunyaev, S. R. (2010). A method and server for predicting damaging missense mutations. Nat Methods, 7 (4), 248-249. doi:

10.1038/nmeth0410-248

Agarwala, Vineeta, Flannick, Jason, Sunyaev, Shamil, Go, T. D. Consortium, \& Altshuler, David. (2013). Evaluating empirical bounds on complex disease genetic architecture. Nature genetics, 45 (12), 1418-1427. doi: 10.1038/ng.2804

Al-Salihi, Mazin, Reichert, Ethan, \& Fitzpatrick, F. A. (2015). Influence of myeloperoxidase on colon tumor occurrence in inflamed versus non-inflamed colons of Apc (Min/+) mice. Redox biology, 6, 218-225. doi:

10.1016/j.redox.2015.07.013

Alter, Blanche P., Rosenberg, Philip S., \& Brody, Lawrence C. (2007). Clinical and molecular features associated with biallelic mutations in FANCD1/BRCA2. Journal of medical genetics, 44 (1), 1-9. doi: 10.1136/jmg.2006.043257

Arakawa, Satoshi, Takahashi, Atsushi, Ashikawa, Kyota, Hosono, Naoya, Aoi, Tomomi, Yasuda, Miho, ... Kubo, Michiaki. (2011). Genome-wide association study identifies two susceptibility loci for exudative age-related macular degeneration in the Japanese population. Nature genetics, 43 (10), 1001-1004. doi: $10.1038 /$ ng.938

Arber, D. A., Orazi, A., Hasserjian, R., Thiele, J., Borowitz, M. J., Le Beau, M. M., ... Vardiman, J. W. (2016). The 2016 revision to the World Health

Organization classification of myeloid neoplasms and acute leukemia. Blood, 127 (20), 2391-2405. doi: 10.1182/blood-2016-03-643544

Astle, W. J., Elding, H., Jiang, T., Allen, D., Ruklisa, D., Mann, A. L., . . Soranzo, N. (2016). The Allelic Landscape of Human Blood Cell Trait Variation and Links to Common Complex Disease. Cell, 167 (5), 1415-1429 e1419. doi:

10.1016/j.cell.2016.10.042

Baker, S. J., Markowitz, S., Fearon, E. R., Willson, J. K., \& Vogelstein, B. (1990). Suppression of human colorectal carcinoma cell growth by wild-type p53.

Science (New York, N.Y.), 249 (4971), 912-915. doi: 10.1126/science.2144057 
Baptista, Renata Lyrio Rafael, Dos Santos, Anna Cláudia Evangelista, Gutiyama, Luciana Mayumi, Solza, Cristiana, \& Zalcberg, Ilana Renault. (2017). Familial Myelodysplastic/Acute Leukemia Syndromes-Myeloid Neoplasms with Germline Predisposition. Frontiers in oncology, 7, 206-206. doi: 10.3389/fonc.2017.00206

Barjesteh van Waalwijk van Doorn-Khosrovani, Sahar, Spensberger, Dominik, de Knegt, Yvonne, Tang, Michelle, Löwenberg, Bob, \& Delwel, Ruud. (2005).

Somatic heterozygous mutations in ETV6 (TEL) and frequent absence of ETV6 protein in acute myeloid leukemia. Oncogene, 24 (25), 4129-4137. doi:

10.1038/sj.onc. 1208588

Betz, Bryan L., \& Hess, Jay L. (2010). Acute myeloid leukemia diagnosis in the 21 st century. Archives of pathology \& laboratory medicine, 134 (10), 1427-1433. doi: 10.1043/2010-0245-RA.1

Blackman, Scott M., Commander, Clayton W., Watson, Christopher, Arcara, Kristin M., Strug, Lisa J., Stonebraker, Jaclyn R., . . Cutting, Garry R. (2013). Genetic modifiers of cystic fibrosis-related diabetes. Diabetes, 62 (10), 36273635. doi: $10.2337 / \mathrm{db} 13-0510$

Bluteau, O., Sebert, M., Leblanc, T., Peffault de Latour, R., Quentin, S., Lainey, E., ... Soulier, J. (2018). A landscape of germ line mutations in a cohort of inherited bone marrow failure patients. Blood, 131 (7), 717-732. doi:

10.1182/blood-2017-09-806489

Boehnke, M. (2000). A look at linkage disequilibrium. Nature genetics, 25 (3), 246-247. doi: 10.1038/76980

Bolton, Kelly L., Tyrer, Jonathan, Song, Honglin, Ramus, Susan J., Notaridou, Maria, Jones, Chris, ... Gayther, Simon A. (2010). Common variants at 19p13 are associated with susceptibility to ovarian cancer. Nature genetics, 42 (10), 880-884. doi: $10.1038 / n g .666$

Bouatia-Naji, Nabila, Bonnefond, Amélie, Cavalcanti-Proença, Christine, Spars $\varnothing$, Thomas, Holmkvist, Johan, Marchand, Marion, ... Froguel, Philippe. (2009). A variant near MTNR1B is associated with increased fasting plasma glucose levels and type 2 diabetes risk. Nature genetics, 41 (1), 89-94. doi: 10.1038/ng.277

Cancer Genome Atlas Research, Network, Weinstein, John N., Collisson, Eric A., Mills, Gordon B., Shaw, Kenna R. Mills, Ozenberger, Brad A., . . Stuart, Joshua M. (2013). The Cancer Genome Atlas Pan-Cancer analysis project. Nature genetics, 45 (10), 1113-1120. doi: 10.1038/ng.2764 
Cancer Genome Atlas Research, Network. (2013). Genomic and epigenomic landscapes of adult de novo acute myeloid leukemia. N Engl J Med, 368 (22), 2059-2074. doi: 10.1056/NEJMoa1301689

Cardoso, S. R., Ryan, G., Walne, A. J., Ellison, A., Lowe, R., Tummala, H., . . . Dokal, I. (2016). Germline heterozygous DDX41 variants in a subset of familial myelodysplasia and acute myeloid leukemia. Leukemia, 30 (10), 2083-2086. doi: 10.1038/leu.2016.124

Care, Rory S., Valk, Peter J. M., Goodeve, Anne C., Abu-Duhier, Faisel M., Geertsma-Kleinekoort, Wendy M. C., Wilson, Giu A., . . Reilly, John T. (2003). Incidence and prognosis of C-KIT and FLT3 mutations in core binding factor (CBF) acute myeloid leukaemias. British journal of haematology, 121 (5), 775777. doi: 10.1046/j.1365-2141.2003.04362.x

Cheah, J. J. C., Hahn, C. N., Hiwase, D. K., Scott, H. S., \& Brown, A. L. (2017). Myeloid neoplasms with germline DDX41 mutation. Int J Hematol, 106 (2), 163174. doi: $10.1007 / \mathrm{s} 12185-017-2260-y$

Choi, Y., \& Chan, A. P. (2015). PROVEAN web server: a tool to predict the functional effect of amino acid substitutions and indels. Bioinformatics, 31 (16), 2745-2747. doi: 10.1093/bioinformatics/btv195

Churpek, J. E., Pyrtel, K., Kanchi, K. L., Shao, J., Koboldt, D., Miller, C. A., . . . Graubert, T. A. (2015). Genomic analysis of germ line and somatic variants in familial myelodysplasia/acute myeloid leukemia. Blood, 126 (22), 2484-2490. doi: 10.1182/blood-2015-04-641100

Cirulli, Elizabeth T., \& Goldstein, David B. (2010). Uncovering the roles of rare variants in common disease through whole-genome sequencing. Nature reviews. Genetics, 11 (6), 415-425. doi: 10.1038/nrg2779

Consortium, G. TEx. (2015). Human genomics. The Genotype-Tissue Expression (GTEx) pilot analysis: multitissue gene regulation in humans. Science, 348 (6235), 648-660. doi: 10.1126/science.1262110

Consortium, Uk K., Walter, Klaudia, Min, Josine L., Huang, Jie, Crooks, Lucy, Memari, Yasin, ... Soranzo, Nicole. (2015). The UK10K project identifies rare variants in health and disease. Nature, 526 (7571), 82-90. doi:

10.1038 /nature14962 
Cooper, GM. (2000). The Cell: A Molecular Approach (2nd ed.): Sunderland (MA): Sinauer Associates.

Dash, A., \& Gilliland, D. G. (2001). Molecular genetics of acute myeloid leukaemia. Best practice \& research. Clinical haematology, 14 (1), 49-64. doi: 10.1053/beha.2000.0115

Deng, Guoren, Lu, You, Zlotnikov, Galina, Thor, Ann D., \& Smith, Helene S. (1996). Loss of Heterozygosity in Normal Tissue Adjacent to Breast Carcinomas. Science, 274 (5295), 2057. doi: 10.1126/science.274.5295.2057

Dickinson, Rachel Emma, Griffin, Helen, Bigley, Venetia, Reynard, Louise N., Hussain, Rafiqul, Haniffa, Muzlifah, ... Collin, Matthew. (2011). Exome sequencing identifies GATA-2 mutation as the cause of dendritic cell, monocyte, B and NK lymphoid deficiency. Blood, 118 (10), 2656-2658. doi: 10.1182/blood2011-06-360313

DiNardo, Courtney D., \& Cortes, Jorge E. (2016). Mutations in AML: prognostic and therapeutic implications. Hematology. American Society of Hematology. Education Program, 2016 (1), 348-355. doi: 10.1182/asheducation-2016.1.348

Ding, G., Liu, F., Feng, C., Xu, J., \& Ding, Q. (2013). Association between the myeloperoxidase gene polymorphisms and the susceptibility to prostate cancer: a case-control study in a Chinese population. [Asociación entre los polimorfismos de genes de mieloperoxidasa y la susceptibilidad a cáncer de próstata: un estudio caso-control en la población de nacionalidad china]. Actas urologicas espanolas, 37 (2), 79-82. doi: 10.1016/j.acuro.2012.03.020

Downward, Julian. (2003). Targeting RAS signalling pathways in cancer therapy. Nature reviews. Cancer, 3 (1), 11-22. doi: 10.1038/nrc969

Druker, Brian J., Guilhot, François, O'Brien, Stephen G., Gathmann, Insa, Kantarjian, Hagop, Gattermann, Norbert, .. . Larson, Richard A. (2006). FiveYear Follow-up of Patients Receiving Imatinib for Chronic Myeloid Leukemia. New England Journal of Medicine, 355 (23), 2408-2417. doi: 10.1056/NEJMoa062867

Farrar, Jason E., Schuback, Heather L., Ries, Rhonda E., Wai, Daniel, Hampton, Oliver A., Trevino, Lisa R., . . . Meshinchi, Soheil. (2016). Genomic Profiling of Pediatric Acute Myeloid Leukemia Reveals a Changing Mutational Landscape from Disease Diagnosis to Relapse. Cancer research, 76 (8), 2197-2205. doi: 10.1158/0008-5472.CAN-15-1015 
Farwell, Kelly D., Shahmirzadi, Layla, El-Khechen, Dima, Powis, Zöe, Chao, Elizabeth C., Tippin Davis, Brigette, ... Tang, Sha. (2015). Enhanced utility of family-centered diagnostic exome sequencing with inheritance model-based analysis: results from 500 unselected families with undiagnosed genetic conditions. Genetics in medicine : official journal of the American College of Medical Genetics, 17 (7), 578-586. doi: 10.1038/gim.2014.154

Fero, M. L., Randel, E., Gurley, K. E., Roberts, J. M., \& Kemp, C. J. (1998). The murine gene p27Kip1 is haplo-insufficient for tumour suppression. Nature, 396 (6707), 177-180. doi: 10.1038/24179

Figueroa, Maria E., Abdel-Wahab, Omar, Lu, Chao, Ward, Patrick S., Patel, Jay, Shih, Alan, ... Melnick, Ari. (2010). Leukemic IDH1 and IDH2 mutations result in a hypermethylation phenotype, disrupt TET2 function, and impair hematopoietic differentiation. Cancer cell, 18 (6), 553-567. doi: 10.1016/j.ccr.2010.11.015

Finlay, C. A., Hinds, P. W., \& Levine, A. J. (1989). The p53 proto-oncogene can act as a suppressor of transformation. Cell, 57 (7), 1083-1093. doi: 10.1016/0092-8674 (89)90045-7

Fisher, Kevin E., Hsu, Amy P., Williams, Christopher L., Sayeed, Hadi, Merritt, Brian Y., Elghetany, M. Tarek, ... Gramatges, Maria Monica. (2017). Somatic mutations in children with GATA2-associated myelodysplastic syndrome who lack other features of GATA2 deficiency. Blood advances, 1 (7), 443-448. doi: 10.1182/bloodadvances.2016002311

Försti, A., Louhelainen, J., Söderberg, M., Wijkström, H., \& Hemminki, K. (2001). Loss of heterozygosity in tumour-adjacent normal tissue of breast and bladder cancer. European Journal of Cancer, 37 (11), 1372-1380. doi: https://doi.org/10.1016/S0959-8049 (01)00118-6

Friend, S. H., Bernards, R., Rogelj, S., Weinberg, R. A., Rapaport, J. M., Albert, D. M., \& Dryja, T. P. (1986). A human DNA segment with properties of the gene that predisposes to retinoblastoma and osteosarcoma. Nature, 323 (6089), 643646. doi: $10.1038 / 323643 \mathrm{a} 0$

Fröhling, Stefan, Scholl, Claudia, Gilliland, D. Gary, \& Levine, Ross L. (2005). Genetics of myeloid malignancies: pathogenetic and clinical implications. Journal of clinical oncology : official journal of the American Society of Clinical Oncology, 23 (26), 6285-6295. doi: 10.1200/JCO.2005.05.010 
Gao, Juehua, Gong, Shunyou, \& Chen, Yi-Hua. (2019). Myeloid Neoplasm With Germline Predisposition: A 2016 Update for Pathologists. Archives of Pathology \& Laboratory Medicine, 143 (1), 13-22. doi: 10.5858/arpa.2017-0194-RA

Godley, L. A., \& Shimamura, A. (2017). Genetic predisposition to hematologic malignancies: management and surveillance. Blood, 130 (4), 424-432. doi: 10.1182/blood-2017-02-735290

Goldin, L. R., Kristinsson, S. Y., Liang, X. S., Derolf, A. R., Landgren, O., \& Bjorkholm, M. (2012). Familial aggregation of acute myeloid leukemia and myelodysplastic syndromes. J Clin Oncol, 30 (2), 179-183. doi:

10.1200/JCO.2011.37.1203

Grimwade, David, Hills, Robert K., Moorman, Anthony V., Walker, Helen, Chatters, Stephen, Goldstone, Anthony H., . . National Cancer Research Institute Adult Leukaemia Working, Group. (2010). Refinement of cytogenetic classification in acute myeloid leukemia: determination of prognostic significance of rare recurring chromosomal abnormalities among 5876 younger adult patients treated in the United Kingdom Medical Research Council trials. Blood, 116 (3), 354-365. doi: 10.1182/blood-2009-11-254441

Grimwade, David, Ivey, Adam, \& Huntly, Brian J. P. (2016). Molecular landscape of acute myeloid leukemia in younger adults and its clinical relevance. Blood, 127 (1), 29-41. doi: 10.1182/blood-2015-07-604496

Grivennikov, Sergei I., Greten, Florian R., \& Karin, Michael. (2010). Immunity, inflammation, and cancer. Cell, 140 (6), 883-899. doi: 10.1016/j.cell.2010.01.025

Grobner, S. N., Worst, B. C., Weischenfeldt, J., Buchhalter, I., Kleinheinz, K., Rudneva, V. A., . . Pfister, S. M. (2018). The landscape of genomic alterations across childhood cancers. Nature, 555 (7696), 321-327. doi:

10.1038 /nature25480

Guidugli, L., Johnson, A. K., Alkorta-Aranburu, G., Nelakuditi, V., Arndt, K., Churpek, J. E., . . Li, Z. (2017). Clinical utility of gene panel-based testing for hereditary myelodysplastic syndrome/acute leukemia predisposition syndromes. Leukemia, 31 (5), 1226-1229. doi: 10.1038/leu.2017.28

Gutmann, David H., Loehr, Allison, Zhang, Yujing, Kim, Joanna, Henkemeyer, Mark, \& Cashen, Amanda. (1999). Haploinsufficiency for the neurofibromatosis 1 (NF1) tumor suppressor results in increased astrocyte proliferation. Oncogene, 18 (31), 4450-4459. doi: 10.1038/sj.onc.1202829 
Hahn, Christopher N., Chong, Chan-Eng, Carmichael, Catherine L., Wilkins, Ella J., Brautigan, Peter J., Li, Xiao-Chun, . . . Scott, Hamish S. (2011). Heritable GATA2 mutations associated with familial myelodysplastic syndrome and acute myeloid leukemia. Nature genetics, 43 (10), 1012-1017. doi: 10.1038/ng.913

Hanahan, Douglas, \& Weinberg, Robert A. (2011). Hallmarks of cancer: the next generation. Cell, 144 (5), 646-674. doi: 10.1016/j.cell.2011.02.013

Hanson, Robert L., Muller, Yunhua L., Kobes, Sayuko, Guo, Tingwei, Bian, Li, Ossowski, Victoria, ... Baier, Leslie J. (2014). A genome-wide association study in American Indians implicates DNER as a susceptibility locus for type 2 diabetes. Diabetes, 63 (1), 369-376. doi: 10.2337/db13-0416

Harada, Hironori, \& Harada, Yuka. (2015). Recent advances in myelodysplastic syndromes: Molecular pathogenesis and its implications for targeted therapies. Cancer science, 106 (4), 329-336. doi: 10.1111/cas.12614

Harada, Hironori, Harada, Yuka, Niimi, Hiromasa, Kyo, Taiichi, Kimura, Akiro, \& Inaba, Toshiya. (2004). High incidence of somatic mutations in the AML1/RUNX1 gene in myelodysplastic syndrome and low blast percentage myeloid leukemia with myelodysplasia. Blood, 103 (6), 2316-2324. doi:

10.1182/blood-2003-09-3074

Harris, N. L., Jaffe, E. S., Diebold, J., Flandrin, G., Muller-Hermelink, H. K., Vardiman, J., . . Bloomfield, C. D. (1999). World Health Organization classification of neoplastic diseases of the hematopoietic and lymphoid tissues: report of the Clinical Advisory Committee meeting-Airlie House, Virginia, November 1997. Journal of clinical oncology : official journal of the American Society of Clinical Oncology, 17 (12), 3835-3849. doi: 10.1200/JCO.1999.17.12.3835

Harrison, P. R., Affara, N., Goldfarb, P. S., Kasturi, K., Yang, Q. S., Lyons, A., ... Nichols, R. (1982). Analysis of red blood cell differentiation. Advances in experimental medicine and biology, 158, 81-88. doi: 10.1007/978-1-4899-52929_9

He, Chunyan, Tamimi, Rulla M., Hankinson, Susan E., Hunter, David J., \& Han, Jiali. (2009). A prospective study of genetic polymorphism in MPO, antioxidant status, and breast cancer risk. Breast cancer research and treatment, 113 (3), 585-594. doi: 10.1007/s10549-008-9962-z 
He, Rong, Wiktor, Anne E., Hanson, Curtis A., Ketterling, Rhett P., Kurtin, Paul J., Van Dyke, Daniel L., ... Reichard, Kaaren K. (2015). Conventional karyotyping and fluorescence in situ hybridization: an effective utilization strategy in diagnostic adult acute myeloid leukemia. American journal of clinical pathology, 143 (6), 873-878. doi: 10.1309/AJCPP6LVMQG4LNCK

Hirsch, Betsy, Shimamura, Akiko, Moreau, Lisa, Baldinger, Shari, Hag-alshiekh, Maha, Bostrom, Bruce, ... D'Andrea, Alan D. (2004). Association of biallelic BRCA2/FANCD1 mutations with spontaneous chromosomal instability and solid tumors of childhood. Blood, 103 (7), 2554-2559. doi: 10.1182/blood-2003-061970

Howlett, Niall G., Taniguchi, Toshiyasu, Olson, Susan, Cox, Barbara, Waisfisz, Quinten, De Die-Smulders, Christine, ... D'Andrea, Alan D. (2002). Biallelic inactivation of BRCA2 in Fanconi anemia. Science (New York, N.Y.), 297 (5581), 606-609. doi: 10.1126/science.1073834

Huang, K. L., Mashl, R. J., Wu, Y., Ritter, D. I., Wang, J., Oh, C., . . Ding, L. (2018). Pathogenic Germline Variants in 10,389 Adult Cancers. Cell, 173 (2), 355-370 e314. doi: 10.1016/j.cell.2018.03.039

International Cancer Genome, Consortium, Hudson, Thomas J., Anderson, Warwick, Artez, Axel, Barker, Anna D., Bell, Cindy, . . Yang, Huanming. (2010). International network of cancer genome projects. Nature, 464 (7291), 993-998. doi: $10.1038 /$ nature08987

Jaiswal, S., Fontanillas, P., Flannick, J., Manning, A., Grauman, P. V., Mar, B. G., . . Ebert, B. L. (2014). Age-related clonal hematopoiesis associated with adverse outcomes. N Engl J Med, 371 (26), 2488-2498. doi:

10.1056/NEJMoa1408617

Joos, S., Haluska, F. G., Falk, M. H., Henglein, B., Hameister, H., Croce, C. M., \& Bornkamm, G. W. (1992). Mapping chromosomal breakpoints of Burkitt's $t$ $(8 ; 14)$ translocations far upstream of c-myc. Cancer research, 52 (23), 65476552 .

Jorde, L. B. (2000). Linkage disequilibrium and the search for complex disease genes. Genome research, 10 (10), 1435-1444. doi: 10.1101/gr.144500

Kaneko, Y., Kobayashi, H., Handa, M., Satake, N., \& Maseki, N. (1997). EWSERG fusion transcript produced by chromosomal insertion in a Ewing sarcoma. Genes, chromosomes \& cancer, 18 (3), 228-231. 
Karczewski, Konrad J., Francioli, Laurent C., Tiao, Grace, Cummings, Beryl B., Alföldi, Jessica, Wang, Qingbo, ... MacArthur, Daniel G. (2019). Variation across 141,456 human exomes and genomes reveals the spectrum of loss-offunction intolerance across human protein-coding genes. bioRxiv, 531210. doi: $10.1101 / 531210$

Kaufman, B., Shapira-Frommer, R., Schmutzler, R. K., Audeh, M. W., Friedlander, M., Balmana, J., . . Domchek, S. M. (2015). Olaparib monotherapy in patients with advanced cancer and a germline BRCA1/2 mutation. J Clin Oncol, 33 (3), 244-250. doi: 10.1200/JCO.2014.56.2728

Kazenwadel, Jan, Secker, Genevieve A., Liu, Yajuan J., Rosenfeld, Jill A., Wildin, Robert S., Cuellar-Rodriguez, Jennifer, ... Harvey, Natasha L. (2012). Loss-of-function germline GATA2 mutations in patients with MDS/AML or MonoMAC syndrome and primary lymphedema reveal a key role for GATA2 in the lymphatic vasculature. Blood, 119 (5), 1283-1291. doi: 10.1182/blood-201108-374363

Kelly, Louise M., \& Gilliland, D. Gary. (2002). Genetics of myeloid leukemias. Annual review of genomics and human genetics, 3, 179-198. doi: 10.1146/annurev.genom.3.032802.115046

Kerber, R. A., \& O'Brien, E. (2005). A cohort study of cancer risk in relation to family histories of cancer in the Utah population database. Cancer, 103 (9), 1906-1915. doi: 10.1002/cncr.20989

Khan, Amjad A., Alsahli, Mohammed A., \& Rahmani, Arshad H. (2018). Myeloperoxidase as an Active Disease Biomarker: Recent Biochemical and Pathological Perspectives. Medical sciences (Basel, Switzerland), 6 (2), 33. doi: $10.3390 /$ medsci6020033

Klein, Robert J., Zeiss, Caroline, Chew, Emily Y., Tsai, Jen-Yue, Sackler, Richard S., Haynes, Chad, .. . Hoh, Josephine. (2005). Complement factor H polymorphism in age-related macular degeneration. Science (New York, N.Y.), 308 (5720), 385-389. doi: 10.1126/science. 1109557

Knudson, A. G. (2001). Two genetic hits (more or less) to cancer. Nature reviews. Cancer, 1 (2), 157-162. doi: 10.1038/35101031

Knudson, A. G., Jr. (1971). Mutation and cancer: statistical study of retinoblastoma. Proceedings of the National Academy of Sciences of the United States of America, 68 (4), 820-823. doi: 10.1073/pnas.68.4.820 
Kosmider, Olivier, \& Moreau-Gachelin, Françoise. (2006). From mice to human: the "two-hit model" of leukemogenesis. Cell cycle (Georgetown, Tex.), 5 (6), 569-570. doi: 10.4161/cc.5.6.2577

Kryukov, Gregory V., Shpunt, Alexander, Stamatoyannopoulos, John A., \& Sunyaev, Shamil R. (2009). Power of deep, all-exon resequencing for discovery of human trait genes. Proceedings of the National Academy of Sciences of the United States of America, 106 (10), 3871-3876. doi: 10.1073/pnas.0812824106

Kurtovic-Kozaric, A., Przychodzen, B., Singh, J., Konarska, M. M., Clemente, M. J., Otrock, Z. K., . . Padgett, R. A. (2014). PRPF8 defects cause missplicing in myeloid malignancies. Leukemia. doi: 10.1038/leu.2014.144

Landau, Dan A., Tausch, Eugen, Taylor-Weiner, Amaro N., Stewart, Chip, Reiter, Johannes G., Bahlo, Jasmin, ... Wu, Catherine J. (2015). Mutations driving CLL and their evolution in progression and relapse. Nature, 526 (7574), 525-530. doi: 10.1038 /nature15395

Lander, E. S., Linton, L. M., Birren, B., Nusbaum, C., Zody, M. C., Baldwin, J., . . International Human Genome Sequencing, Consortium. (2001). Initial sequencing and analysis of the human genome. Nature, 409 (6822), 860-921. doi: $10.1038 / 35057062$

Landrum, Melissa J., Lee, Jennifer M., Benson, Mark, Brown, Garth R., Chao, Chen, Chitipiralla, Shanmuga, ... Maglott, Donna R. (2018). ClinVar: improving access to variant interpretations and supporting evidence. Nucleic acids research, 46 (D1), D1062-D1067. doi: 10.1093/nar/gkx1153

Leone, Maurizio A., Barizzone, Nadia, Esposito, Federica, Lucenti, Ausiliatrice, Harbo, Hanne F., Goris, An, . . D'Alfonso, Sandra. (2013). Association of genetic markers with CSF oligoclonal bands in multiple sclerosis patients. PloS one, 8 (6), e64408-e64408. doi: 10.1371/journal.pone.0064408

Leung, Wai K., Yu, Jun, Ng, Enders K. W., To, Ka Fai, Ma, Po K., Lee, Tin Lap, ... Sung, Joseph J. Y. (2001). Concurrent hypermethylation of multiple tumor-related genes in gastric carcinoma and adjacent normal tissues. Cancer, 91 (12), 2294-2301. doi: 10.1002/1097-0142 (20010615)91:12<2294::aidcncr1261>3.0.co;2-g

Lewinsohn, Maya, Brown, Anna L., Weinel, Luke M., Phung, Connie, Rafidi, George, Lee, Ming K., . . . Scott, Hamish S. (2016). Novel germ line DDX41 
mutations define families with a lower age of MDS/AML onset and lymphoid malignancies. Blood, 127 (8), 1017-1023. doi: 10.1182/blood-2015-10-676098

Li, H., \& Durbin, R. (2010). Fast and accurate long-read alignment with BurrowsWheeler transform. Bioinformatics, 26 (5), 589-595. doi:

10.1093/bioinformatics/btp698

Li, H., Handsaker, B., Wysoker, A., Fennell, T., Ruan, J., Homer, N., . . Genome Project Data Processing, Subgroup. (2009). The Sequence Alignment/Map format and SAMtools. Bioinformatics, 25 (16), 2078-2079. doi:

10.1093/bioinformatics/btp352

Li, Ruijuan, Sobreira, Nara, Witmer, P. Dane, Pratz, Keith W., \& Braunstein, Evan M. (2016). Two novel germline DDX41 mutations in a family with inherited myelodysplasia/acute myeloid leukemia. Haematologica, 101 (6), e228-e231. doi: 10.3324/haematol.2015.139790

Lichtenstein, P., Holm, N. V., Verkasalo, P. K., lliadou, A., Kaprio, J., Koskenvuo, M., ... Hemminki, K. (2000). Environmental and heritable factors in the causation of cancer--analyses of cohorts of twins from Sweden, Denmark, and Finland. N Engl J Med, 343 (2), 78-85. doi: 10.1056/NEJM200007133430201 Liu, Jinghua, Lewinger, Juan Pablo, Gilliland, Frank D., Gauderman, W. James, \& Conti, David V. (2013). Confounding and heterogeneity in genetic association studies with admixed populations. American journal of epidemiology, 177 (4), 351-360. doi: 10.1093/aje/kws234

Loizidou, Maria A., Hadjisavvas, Andreas, Tanteles, George A., SpanouAristidou, Elena, Kyriacou, Kyriacos, \& Christophidou-Anastasiadou, Violetta. (2016). Fanconi anemia-D1 due to homozygosity for the BRCA2 gene Cypriot founder mutation: A case report. Oncology letters, 11 (1), 471-473. doi: 10.3892/ol.2015.3852

Luo, Xi, Feurstein, Simone, Mohan, Shruthi, Porter, Christopher C., Jackson, Sarah A., Keel, Sioban, ... Godley, Lucy A. (2019). ClinGen Myeloid Malignancy Variant Curation Expert Panel recommendations for germline RUNX1 variants. Blood advances, 3 (20), 2962-2979. doi:

10.1182/bloodadvances.2019000644

Ma, Ronald Ching Wan, Lee, Heung Man, Lam, Vincent Kwok Lim, Tam, Claudia Ha Ting, Ho, Janice Siu Ka, Zhao, Hai-Lu, . . . Chan, Juliana Chung Ngor. (2014). Familial young-onset diabetes, pre-diabetes and cardiovascular disease 
are associated with genetic variants of DACH1 in Chinese. PloS one, 9 (1), e84770-e84770. doi: 10.1371/journal.pone.0084770

Machiela, M. J., \& Chanock, S. J. (2018). LDassoc: an online tool for interactively exploring genome-wide association study results and prioritizing variants for functional investigation. Bioinformatics, 34 (5), 887-889. doi: 10.1093/bioinformatics/btx561

Machiela, Mitchell J., \& Chanock, Stephen J. (2018). LDassoc: an online tool for interactively exploring genome-wide association study results and prioritizing variants for functional investigation. Bioinformatics (Oxford, England), 34 (5), 887-889. doi: 10.1093/bioinformatics/bt×561

Makishima, H., Yoshida, K., Nguyen, N., Przychodzen, B., Sanada, M., Okuno, Y., . . Maciejewski, J. P. (2013). Somatic SETBP1 mutations in myeloid malignancies. Nat Genet, 45 (8), 942-946. doi: 10.1038/ng.2696

Makishima, H., Yoshizato, T., Nannya, Y., Momozawa, Y., Atsuta, Y., Shiozawa, Y., ... Ogawa, S. (2018). Novel and Significant Impact of Germline Variants Predisposed to Pathogenic Somatic Mutations and Loss of Heterozygosity (LOH) in Myelodysplastic Syndromes (MDS) and Clonal Hematopoiesis of Indeterminate Potential (CHIP). Blood, 132 (Suppl 1), 108.

Makishima, H., Yoshizato, T., Yoshida, K., Sekeres, M. A., Radivoyevitch, T., Suzuki, H., . . Maciejewski, J. P. (2017). Dynamics of clonal evolution in myelodysplastic syndromes. Nat Genet, 49 (2), 204-212. doi: 10.1038/ng.3742

Manolio, Teri A., Collins, Francis S., Cox, Nancy J., Goldstein, David B., Hindorff, Lucia A., Hunter, David J., . . Visscher, Peter M. (2009). Finding the missing heritability of complex diseases. Nature, 461 (7265), 747-753. doi:

10.1038 /nature08494

Marchetti, C., Patriarca, P., Solero, G. P., Baralle, F. E., \& Romano, M. (2004). Genetic characterization of myeloperoxidase deficiency in Italy. Hum Mutat, 23 (5), 496-505. doi: 10.1002/humu.20027

Martinelli-Boneschi, Filippo, Esposito, Federica, Brambilla, Paola, Lindström, Eva, Lavorgna, Giovanni, Stankovich, Jim, ... Comi, Giancarlo. (2012). A genome-wide association study in progressive multiple sclerosis. Multiple sclerosis (Houndmills, Basingstoke, England), 18 (10), 1384-1394. doi: $10.1177 / 1352458512439118$ 
Maung, Kyaw Ze Ya, Leo, Paul J., Bassal, Mahmoud, Casolari, Debora A., Gray, James X., Bray, Sarah C., . . Gonda, Thomas J. (2018). Rare variants in Fanconi anemia genes are enriched in acute myeloid leukemia. Blood Cancer Journal, 8 (6), 50. doi: 10.1038/s41408-018-0090-7

McCarthy, Shane, Das, Sayantan, Kretzschmar, Warren, Delaneau, Olivier, Wood, Andrew R., Teumer, Alexander, ... the Haplotype Reference, Consortium. (2016). A reference panel of 64,976 haplotypes for genotype imputation. Nature Genetics, 48 (10), 1279-1283. doi: 10.1038/ng.3643

McKenna, A., Hanna, M., Banks, E., Sivachenko, A., Cibulskis, K., Kernytsky, A., ... DePristo, M. A. (2010). The Genome Analysis Toolkit: a MapReduce framework for analyzing next-generation DNA sequencing data. Genome Res, 20 (9), 1297-1303. doi: 10.1101/gr.107524.110

Michaud, J., Wu, F., Osato, M., Cottles, G. M., Yanagida, M., Asou, N., . . Scott, H. S. (2002). In vitro analyses of known and novel RUNX1/AML1 mutations in dominant familial platelet disorder with predisposition to acute myelogenous leukemia: implications for mechanisms of pathogenesis. Blood, 99 (4), 13641372.

Moreau-Gachelin, Françoise. (2006). Lessons from models of murine erythroleukemia to acute myeloid leukemia (AML): proof-of-principle of cooperativity in AML. Haematologica, 91 (12), 1644-1652.

Murati, Anne, Brecqueville, Mandy, Devillier, Raynier, Mozziconacci, MarieJoelle, Gelsi-Boyer, Véronique, \& Birnbaum, Daniel. (2012). Myeloid malignancies: mutations, models and management. BMC cancer, 12, 304-304. doi: 10.1186/1471-2407-12-304

Naj, Adam C., Scott, William K., Courtenay, Monique D., Cade, William H., Schwartz, Stephen G., Kovach, Jaclyn L., . . Pericak-Vance, Margaret A. (2013). Genetic factors in nonsmokers with age-related macular degeneration revealed through genome-wide gene-environment interaction analysis. Annals of human genetics, 77 (3), 215-231. doi: 10.1111/ahg.12011

National Institutes of Health, U.S. Department of Health and Human Services. ( 2016). NIH Stem Cell Information Home Page. In Stem Cell Information. .

Nauseef, W. M., Brigham, S., \& Cogley, M. (1994). Hereditary myeloperoxidase deficiency due to a missense mutation of arginine 569 to tryptophan. The Journal of biological chemistry, 269 (2), 1212-1216. 
Ng, P. C., \& Henikoff, S. (2003). SIFT: Predicting amino acid changes that affect protein function. Nucleic Acids Res, 31 (13), 3812-3814.

Noetzli, Leila, Lo, Richard W., Lee-Sherick, Alisa B., Callaghan, Michael, Noris, Patrizia, Savoia, Anna, ... Di Paola, Jorge. (2015). Germline mutations in ETV6 are associated with thrombocytopenia, red cell macrocytosis and predisposition to lymphoblastic leukemia. Nature genetics, 47 (5), 535-538. doi:

$10.1038 /$ ng.3253

Nordborg, Magnus, \& Tavaré, Simon. (2002). Linkage disequilibrium: what history has to tell us. Trends in genetics : TIG, 18 (2), 83-90. doi: 10.1016/s01689525 (02)02557-x

Noris, P., Perrotta, S., Seri, M., Pecci, A., Gnan, C., Loffredo, G., . . Savoia, A. (2011). Mutations in ANKRD26 are responsible for a frequent form of inherited thrombocytopenia: analysis of 78 patients from 21 families. Blood, 117 (24), 6673-6680. doi: 10.1182/blood-2011-02-336537

Offit, Kenneth, Levran, Orna, Mullaney, Brian, Mah, Katherine, Nafa, Khedoudja, Batish, Sat Dev, ... Auerbach, Arleen D. (2003). Shared genetic susceptibility to breast cancer, brain tumors, and Fanconi anemia. Journal of the National Cancer Institute, 95 (20), 1548-1551. doi: 10.1093/jnci/djg072

Pabst, T., \& Mueller, B. U. (2009). Complexity of CEBPA dysregulation in human acute myeloid leukemia. Clin Cancer Res, 15 (17), 5303-5307. doi:

10.1158/1078-0432.CCR-08-2941

Papaemmanuil, E., Gerstung, M., Bullinger, L., Gaidzik, V. I., Paschka, P., Roberts, N. D., . . Campbell, P. J. (2016). Genomic Classification and Prognosis in Acute Myeloid Leukemia. N Engl J Med, 374 (23), 2209-2221. doi: 10.1056/NEJMoa1516192

Park, Ju-Hyun, Gail, Mitchell H., Weinberg, Clarice R., Carroll, Raymond J., Chung, Charles C., Wang, Zhaoming, ... Chatterjee, Nilanjan. (2011).

Distribution of allele frequencies and effect sizes and their interrelationships for common genetic susceptibility variants. Proceedings of the National Academy of Sciences of the United States of America, 108 (44), 18026-18031. doi: 10.1073/pnas. 1114759108

Park, Sang Hyuk, Chi, Hyun-Sook, Min, Sook-Kyung, Park, Borae G., Jang, Seongsoo, \& Park, Chan-Jeoung. (2011). Prognostic impact of c-KIT mutations 
in core binding factor acute myeloid leukemia. Leukemia research, 35 (10), 13761383. doi: 10.1016/j.leukres.2011.06.003

Pirahmadian, Alireza. (2016). transgenic natural killer cells.

Polprasert, C., Schulze, I., Sekeres, M. A., Makishima, H., Przychodzen, B., Hosono, N., . . Maciejewski, J. P. (2015). Inherited and Somatic Defects in DDX41 in Myeloid Neoplasms. Cancer Cell, 27 (5), 658-670. doi:

10.1016/j.ccell.2015.03.017

Polprasert, Chantana, Schulze, Isabell, Sekeres, Mikkael A., Makishima, Hideki, Przychodzen, Bartlomiej, Hosono, Naoko, . . Maciejewski, Jaroslaw P. (2015). Inherited and Somatic Defects in DDX41 in Myeloid Neoplasms. Cancer cell, 27 (5), 658-670. doi: 10.1016/j.ccell.2015.03.017

Pritchard, C. C., Mateo, J., Walsh, M. F., De Sarkar, N., Abida, W., Beltran, H., ... Nelson, P. S. (2016). Inherited DNA-Repair Gene Mutations in Men with Metastatic Prostate Cancer. N Engl J Med, 375 (5), 443-453. doi: 10.1056/NEJMoa1603144

Pritchard, Jonathan K., \& Przeworski, Molly. (2001). Linkage Disequilibrium in Humans: Models and Data. The American Journal of Human Genetics, 69 (1), 114. doi: https://doi.org/10.1086/321275

Purrington, Kristen S., Slager, Susan, Eccles, Diana, Yannoukakos, Drakoulis, Fasching, Peter A., Miron, Penelope, ... Couch, Fergus J. (2014). Genomewide association study identifies 25 known breast cancer susceptibility loci as risk factors for triple-negative breast cancer. Carcinogenesis, 35 (5), 1012-1019. doi: $10.1093 /$ carcin/bgt404

Rahman, N. (2014). Realizing the promise of cancer predisposition genes. Nature, 505 (7483), 302-308. doi: 10.1038/nature12981

Rentzsch, P., Witten, D., Cooper, G. M., Shendure, J., \& Kircher, M. (2018). CADD: predicting the deleteriousness of variants throughout the human genome. Nucleic Acids Res. doi: 10.1093/nar/gky1016

Reva, B., Antipin, Y., \& Sander, C. (2011). Predicting the functional impact of protein mutations: application to cancer genomics. Nucleic Acids Res, 39 (17), e118. doi: $10.1093 / \mathrm{nar} / \mathrm{gkr} 407$ 
Richards, Sue, Aziz, Nazneen, Bale, Sherri, Bick, David, Das, Soma, GastierFoster, Julie, ... Committee, Acmg Laboratory Quality Assurance. (2015). Standards and guidelines for the interpretation of sequence variants: a joint consensus recommendation of the American College of Medical Genetics and Genomics and the Association for Molecular Pathology. Genetics in medicine : official journal of the American College of Medical Genetics, 17 (5), 405-424. doi: 10.1038/gim.2015.30

Richkind, K. E., Loew, T., Meisner, L., Harris, C., \& Wason, D. (1998). Identical cytogenetic clones and clonal evolution in pediatric monozygotic twins with acute myeloid leukemia: presymptomatic disease detection by interphase fluorescence in situ hybridization and review of the literature. J Pediatr Hematol Oncol, 20 (3), 264-267.

Robinson, J. T., Thorvaldsdottir, H., Winckler, W., Guttman, M., Lander, E. S., Getz, G., \& Mesirov, J. P. (2011). Integrative genomics viewer. Nat Biotechnol, 29 (1), 24-26. doi: 10.1038/nbt.1754

Rous, P. (1911). A SARCOMA OF THE FOWL TRANSMISSIBLE BY AN AGENT SEPARABLE FROM THE TUMOR CELLS. The Journal of experimental medicine, 13 (4), 397-411. doi: 10.1084/jem.13.4.397

Salesse, Stephanie, \& Verfaillie, Catherine M. (2002). BCR/ABL: from molecular mechanisms of leukemia induction to treatment of chronic myelogenous leukemia. Oncogene, 21 (56), 8547-8559. doi: 10.1038/sj.onc.1206082

Scarpa, A., Chang, D. K., Nones, K., Corbo, V., Patch, A. M., Bailey, P., . . . Grimmond, S. M. (2017). Whole-genome landscape of pancreatic neuroendocrine tumours. Nature, 543 (7643), 65-71. doi: 10.1038/nature21063

Schmidt, T., Samaras, P., Frejno, M., Gessulat, S., Barnert, M., Kienegger, H., ... Wilhelm, M. (2018). ProteomicsDB. Nucleic Acids Res, 46 (D1), D1271D1281. doi: 10.1093/nar/gkx1029

Schnittger, S., Bacher, U., Kern, W., Alpermann, T., Haferlach, C., \& Haferlach, T. (2011). Prognostic impact of FLT3-ITD load in NPM1 mutated acute myeloid leukemia. Leukemia, 25 (8), 1297-1304. doi: 10.1038/leu.2011.97

Schnittger, Susanne, Schoch, Claudia, Dugas, Martin, Kern, Wolfgang, Staib, Peter, Wuchter, Christian, ... Hiddemann, Wolfgang. (2002). Analysis of FLT3 length mutations in 1003 patients with acute myeloid leukemia: correlation to cytogenetics, FAB subtype, and prognosis in the AMLCG study and usefulness 
as a marker for the detection of minimal residual disease. Blood, 100 (1), 59-66. doi: 10.1182/blood.v100.1.59

Schrader, K. A., Cheng, D. T., Joseph, V., Prasad, M., Walsh, M., Zehir, A., . . Robson, M. (2016). Germline Variants in Targeted Tumor Sequencing Using Matched Normal DNA. JAMA Oncol, 2 (1), 104-111. doi:

10.1001/jamaoncol.2015.5208

Schwartz, J. R., Ma, J., Lamprecht, T., Walsh, M., Wang, S., Bryant, V., . . Klco, J. M. (2017). The genomic landscape of pediatric myelodysplastic syndromes. Nat Commun, 8 (1), 1557. doi: 10.1038/s41467-017-01590-5

Schwarz, J. M., Cooper, D. N., Schuelke, M., \& Seelow, D. (2014).

MutationTaster2: mutation prediction for the deep-sequencing age. Nat Methods, 11 (4), 361-362. doi: 10.1038/nmeth.2890

Song, W. J., Sullivan, M. G., Legare, R. D., Hutchings, S., Tan, X., Kufrin, D., . . . Gilliland, D. G. (1999). Haploinsufficiency of CBFA2 causes familial thrombocytopenia with propensity to develop acute myelogenous leukaemia. Nat Genet, 23 (2), 166-175. doi: 10.1038/13793

Sood, Raman, Kamikubo, Yasuhiko, \& Liu, Paul. (2017). Role of RUNX1 in hematological malignancies. Blood, 129 (15), 2070-2082. doi: 10.1182/blood2016-10-687830

Speed, Doug, Hemani, Gibran, Johnson, Michael R., \& Balding, David J. (2012). Improved heritability estimation from genome-wide SNPs. American journal of human genetics, 91 (6), 1011-1021. doi: 10.1016/j.ajhg.2012.10.010

Stehelin, D., Varmus, H. E., Bishop, J. M., \& Vogt, P. K. (1976). DNA related to the transforming gene (s) of avian sarcoma viruses is present in normal avian DNA. Nature, 260 (5547), 170-173. doi: 10.1038/260170a0

Stieglitz, Elliot, Taylor-Weiner, Amaro N., Chang, Tiffany Y., Gelston, Laura C., Wang, Yong-Dong, Mazor, Tali, ... Loh, Mignon L. (2015). The genomic landscape of juvenile myelomonocytic leukemia. Nature genetics, 47 (11), 13261333. doi: $10.1038 / \mathrm{ng} .3400$

Strom, S. S., Gu, Y., Gruschkus, S. K., Pierce, S. A., \& Estey, E. H. (2005). Risk factors of myelodysplastic syndromes: a case-control study. Leukemia, 19 (11), 1912-1918. doi: 10.1038/sj.leu.2403945 
Sun, B. B., Maranville, J. C., Peters, J. E., Stacey, D., Staley, J. R., Blackshaw, J., . . Butterworth, A. S. (2018). Genomic atlas of the human plasma proteome. Nature, 558 (7708), 73-79. doi: 10.1038/s41586-018-0175-2

Swerdlow, S. H. (2008). WHO classification of tumours of haematopoietic and lymphoid tissues. WHO classification of tumours, 22008, 439.

Tapper, W., Jones, A. V., Kralovics, R., Harutyunyan, A. S., Zoi, K., Leung, W., ... Cross, N. C. (2015). Genetic variation at MECOM, TERT, JAK2 and HBS1L-MYB predisposes to myeloproliferative neoplasms. Nat Commun, 6 , 6691. doi: 10.1038/ncomms7691

Taylor-Weiner, Amaro, Stewart, Chip, Giordano, Thomas, Miller, Mendy, Rosenberg, Mara, Macbeth, Alyssa, ... Getz, Gad. (2018). DeTiN: overcoming tumor-in-normal contamination. Nature Methods, 15 (7), 531-534. doi: 10.1038/s41592-018-0036-9

Thiede, Christian, Steudel, Christine, Mohr, Brigitte, Schaich, Markus, Schäkel, Ulrike, Platzbecker, Uwe, . . Illmer, Thomas. (2002). Analysis of FLT3activating mutations in 979 patients with acute myelogenous leukemia: association with $\mathrm{FAB}$ subtypes and identification of subgroups with poor prognosis. Blood, 99 (12), 4326-4335. doi: 10.1182/blood.v99.12.4326

Tyner, J. W., Tognon, C. E., Bottomly, D., Wilmot, B., Kurtz, S. E., Savage, S. L., . . . Druker, B. J. (2018). Functional genomic landscape of acute myeloid leukaemia. Nature, 562 (7728), 526-531. doi: 10.1038/s41586-018-0623-z VanderVeen, L. A., Hashim, M. F., Nechev, L. V., Harris, T. M., Harris, C. M., \& Marnett, L. J. (2001). Evaluation of the mutagenic potential of the principal DNA adduct of acrolein. The Journal of biological chemistry, 276 (12), 9066-9070. doi: 10.1074/jbc.M008900200

Vardiman, James W., Thiele, Jüergen, Arber, Daniel A., Brunning, Richard D., Borowitz, Michael J., Porwit, Anna, . . Bloomfield, Clara D. (2009). The 2008 revision of the World Health Organization (WHO) classification of myeloid neoplasms and acute leukemia: rationale and important changes. Blood, 114 (5), 937-951. doi: 10.1182/blood-2009-03-209262

Venter, J. C., Adams, M. D., Myers, E. W., Li, P. W., Mural, R. J., Sutton, G. G., . . Zhu, X. (2001). The sequence of the human genome. Science (New York, N.Y.), 291 (5507), 1304-1351. doi: 10.1126/science.1058040 
Wagner, John E., Tolar, Jakub, Levran, Orna, Scholl, Thomas, Deffenbaugh, Amie, Satagopan, Jaya, ... Auerbach, Arleen D. (2004). Germline mutations in BRCA2: shared genetic susceptibility to breast cancer, early onset leukemia, and Fanconi anemia. Blood, 103 (8), 3226-3229. doi: 10.1182/blood-2003-09-3138

Walker, Logan C., Stevens, Jane, Campbell, Hamish, Corbett, Rob, Spearing, Ruth, Heaton, David, ... Ganly, Peter. (2002). A novel inherited mutation of the transcription factor RUNX1 causes thrombocytopenia and may predispose to acute myeloid leukaemia. British journal of haematology, 117 (4), 878-881. doi: 10.1046/j.1365-2141.2002.03512.x

Wang, K., Li, M., \& Hakonarson, H. (2010). ANNOVAR: functional annotation of genetic variants from high-throughput sequencing data. Nucleic Acids Res, 38 (16), e164. doi: $10.1093 /$ nar/gkq603

Wang, Qinrong, Dong, Shasha, Yao, Hong, Wen, Lijun, Qiu, Huiying, Qin, Llili, ... Chen, Suning. (2014). ETV6 mutation in a cohort of 970 patients with hematologic malignancies. Haematologica, 99 (10), e176-e178. doi: 10.3324/haematol.2014.104406

Wang, Weidong. (2007). Emergence of a DNA-damage response network consisting of Fanconi anaemia and BRCA proteins. Nature reviews. Genetics, 8 (10), 735-748. doi: 10.1038/nrg2159

Wei, Lei, Papanicolau-Sengos, Antonios, Liu, Song, Wang, Jianmin, Conroy, Jeffrey M., Glenn, Sean T., ... Morrison, Carl D. (2016). Pitfalls of improperly procured adjacent non-neoplastic tissue for somatic mutation analysis using next-generation sequencing. BMC Medical Genomics, 9 (1), 64. doi:

10.1186/s12920-016-0226-1

Welch, John S, Ley, Timothy J, Link, Daniel C, Miller, Christopher A, Larson, David E, Koboldt, Daniel C, ... Wilson, Richard K. (2012). The Origin and Evolution of Mutations in Acute Myeloid Leukemia. Cell, 150 (2), 264-278. doi: https://doi.org/10.1016/j.cell.2012.06.023

Won, Hong-Hee, Lee, Jeeyun, Park, Joon Oh, Park, Young Suk, Lim, Ho Yeong, Kang, Won Ki, ... Park, Se Hoon. (2012). Polymorphic markers associated with severe oxaliplatin-induced, chronic peripheral neuropathy in colon cancer patients. Cancer, 118 (11), 2828-2836. doi: 10.1002/cncr.26614

Wright, Fred A., Strug, Lisa J., Doshi, Vishal K., Commander, Clayton W., Blackman, Scott M., Sun, Lei, . . Cutting, Garry R. (2011). Genome-wide 
association and linkage identify modifier loci of lung disease severity in cystic fibrosis at $11 \mathrm{p} 13$ and 20q13.2. Nature genetics, 43 (6), 539-546. doi: $10.1038 / \mathrm{ng} .838$

Yoshimoto, M., Caminada De Toledo, S. R., Monteiro Caran, E. M., de Seixas, M. T., de Martino Lee, M. L., de Campos Vieira Abib, S., . . Anderson Duffles Andrade, J. (1999). MYCN gene amplification. Identification of cell populations containing double minutes and homogeneously staining regions in neuroblastoma tumors. The American journal of pathology, 155 (5), 1439-1443. doi: 10.1016/S0002-9440 (10)65457-0

Zhang, J., Walsh, M. F., Wu, G., Edmonson, M. N., Gruber, T. A., Easton, J., . . . Downing, J. R. (2015). Germline Mutations in Predisposition Genes in Pediatric Cancer. N Engl J Med, 373 (24), 2336-2346. doi: 10.1056/NEJMoa1508054

Zhang, M. Y., Churpek, J. E., Keel, S. B., Walsh, T., Lee, M. K., Loeb, K. R., . . . Shimamura, A. (2015). Germline ETV6 mutations in familial thrombocytopenia and hematologic malignancy. Nat Genet, 47 (2), 180-185. doi: 10.1038/ng.3177

Zhang, Michael Y., Churpek, Jane E., Keel, Siobán B., Walsh, Tom, Lee, Ming K., Loeb, Keith R., . . . Shimamura, Akiko. (2015). Germline ETV6 mutations in familial thrombocytopenia and hematologic malignancy. Nature genetics, 47 (2), 180-185. doi: 10.1038/ng.3177

Zhang, Renliang, Brennan, Marie-Luise, Fu, Xiaoming, Aviles, Ronnier J., Pearce, Gregory L., Penn, Marc S., . . Hazen, Stanley L. (2001). Association Between Myeloperoxidase Levels and Risk of Coronary Artery Disease. JAMA, 286 (17), 2136-2142. doi: 10.1001/jama.286.17.2136

Zuk, Or, Schaffner, Stephen F., Samocha, Kaitlin, Do, Ron, Hechter, Eliana, Kathiresan, Sekar, ... Lander, Eric S. (2014). Searching for missing heritability: Designing rare variant association studies. Proceedings of the National Academy of Sciences, 111 (4), E455-E464. doi: 10.1073/pnas.1322563111

Zurawel, R. H., Allen, C., Wechsler-Reya, R., Scott, M. P., \& Raffel, C. (2000). Evidence that haploinsufficiency of Ptch leads to medulloblastoma in mice. Genes, chromosomes \& cancer, 28 (1), 77-81. 